\title{
DEVELOPMENT OF A PHOSPHOLIPID ENCAPSULATION PROCESS FOR QUANTUM DOTS TO BE USED IN BIOLOGIC APPLICATIONS
}

\author{
A Thesis \\ presented to \\ the Faculty of California Polytechnic State University, \\ of San Luis Obispo
}

In Partial Fulfillment
of the Requirements for the Degree
Master of Science in General Engineering - Materials Engineering

by

Logan Michael Grimes

June 2014 
(C) 2014

Logan M. Grimes

ALL RIGHTS RESERVED 


\section{COMMITTEE MEMBERSHIP}

TITLE:

AUTHOR:

DATE SUBMITTED:

COMMITTEE CHAIR:

COMMITTEE MEMBER:

COMMITTEE MEMBER:

COMMITTEE MEMBER:
Development of a Phospholipid

Encapsulation Process for Quantum

Dots to be used in Biologic

Applications

Logan Michael Grimes

June 13, 2014

Richard Savage, PhD

Professor, Biomedical \& General

Engineering

Philip Costanzo, PhD

Associate Professor of Chemistry \&

Biochemistry

Rafael Jimenez-Flores, PhD

Professor, Dairy Science

Lily Laiho, PhD

Professor, Biomedical \& General

Engineering 


\begin{abstract}
Development of a Phospholipid Encapsulation Process for Quantum Dots to be used in Biologic Applications

Logan Michael Grimes
\end{abstract}

The American Cancer Society predicts that $1,665,540$ people will be diagnosed with cancer, and 585,720 people will die from cancer in 2014 . One of the most common types of cancer in the United States is skin cancer. Melanoma alone is predicted to account for 10,000 of the cancer related deaths in 2014. As a highly mobile and aggressive form of cancer, melanoma is difficult to fight once it has metastasized through the body. Early detection in such varieties of cancer is critical in improving survival rates in afflicted patients. Present methods of detection rely on visual examination of suspicious regions of tissue via various forms of biopsies. Accurate assessment of cancerous cells via this method are subjective, and often unreliable in the early stages of cancer formation when only few cancer cells are forming. With fewer cancer cells, it is less likely that a cancer cell will appear in a biopsied tissue. This leads to a lower detection rate, even when cancer is present. This lack of detection when cancer is in fact present is referred to as a false negative. False negatives can have a highly detrimental effect on treating the cancer as soon as possible. More accurate methods of detecting cancer in early stages, in a nonsubjective form would alleviate these problems. A proposed alternative to visual examination of biopsied legions is to utilize fluorescent nanocrystalline biomarker constructs to directly attach to the abnormal markers found on cancerous tissues. 
Quantum dots (QDs) are hydrophobic nanoscale crystals composed of semiconducting materials which fluoresce when exposed to specific wavelengths of radiation, most commonly in the form of an ultraviolet light source. The QD constructs generated were composed of cadmium-selenium (CdSe) cores encapsulated with zinc-sulfide (ZnS) shells. These QDs were then encapsulated with phospholipids in an effort to create a hydrophilic particle which could interact with polar fluids as found within the human body. The goal of this thesis is to develop a method for the solubilization, encapsulation, and initial functionalization of CdSe/ZnS QDs. The first stage of this thesis focused on the generation of CdSe/ZnS QDs and the fluorescence differences between unshelled and shelled QDs. The second stage focused on utilizing the shelled QDs to generate hydrophilic constructs by utilizing phospholipids to bind with the QDs. Analysis via spectroscopy was performed in an effort to characterize the difference in QDs both prior to and after the encapsulation process. The method generated provides insight on fluorescence trends and the encapsulation of QDs in polar substances. Future research focusing on the repeatability of the process, introducing the QD constructs to a biological material, and eventual interaction with cancer cells are the next steps in generating a new technique to target and reveal skin cancer cells in the earliest possible stages without using a biopsy. 


\section{ACKNOWLEDGMENTS}

I would like to thank Dr. Philip Costanzo for his significant input on the processing methodology. Your expertise on quantum dots was invaluable in my research experience, and I greatly valued the inputs given. Without your insight and constructive criticism, I would have remained ill-informed in my assumptions overall. I consider myself far more experienced from my time conversing with you. I would also like to thank Dr. Lily Laiho. Your experience helped me through the early stages of my inexperience working with quantum dots. Though busy, you created time in your schedule to help me understand the core work of my thesis. I would also like to thank Dr. Rafael Jimenez. Your critiques helped me to reevaluate critical pieces of my thesis, and improve them drastically. Your support both inside and out of the laboratory has been greatly appreciated. I always seemed to leave your office without an increased sense of purpose and positive outlook. I would finally like to thank Dr. Richard Savage. In my thesis work, my other academic ventures, and in my professional life, you have acted as a mentor for me. Both in the earliest stages of my studies of materials engineering in introductory courses, and in the highest graduate level classes and technical laboratories, you have helped to expand my knowledge and given me valuable insights. I do not think any professor at Cal Poly has shaped my experience more, and I feel privileged for the experience. I would like to thank all of these professors for the significant time and effort spent working with me on this thesis to accelerate the project in a shortened timeframe. Without your substantial efforts, I would not be in the same place I am now. 
I would also like to thank my peers for their contributions. Fellow graduate student, and soon to be Cal Poly alumnus, Harry Lafferty for his insights at various points through my thesis, as well as for helping me by providing a data extraction program for data analysis. You have been a great friend through my time at Cal Poly. I would also like to thank Evan Cate. Though less directly involved with the technical aspects, you have helped my work when you have had no personal investment. I would also like to thank Robert Kobara for teaching me the synthesis and shelling methods of quantum dots. Your experience with the project was indispensable in my experience with my thesis. Your patience in my initial learning was greatly appreciated. I would also like to thank previous students who helped to develop the quantum dot program at $\mathrm{Cal}$ Poly, including but not limited to Laura Sparks, Aaron Lichtner, and Joshua Angell. Without your efforts, I would have missed an amazing experience in such an interesting field of research.

I would finally like to thank my family. All words will fall short in expressing my gratitude. My grandparents, aunts, uncles, and cousins have been nothing but supportive of my efforts. I would like to thank my parents especially. Mom, your calm supporting push has both kept me functioning in the highest stress situations, and kept me progressing forward with a supreme belief in my own abilities, understanding that "impossible is nothing" where dedicate myself. Dad, I look to your experiences to draw my own power of will. Through your support, occasional tough-love, and through seeing what you have achieved through your effort, I have been able to progress to where I am today. You both have acted as 
constant inspirations and heroes in my life. Finally I would like to thank my sister Lauren Grimes. I do not think you understand how often I look up to you for inspiration. In every significant transition in my life, you have blazed a trail in front of me. Every grade level, changing to a new high school, and graduating college all felt much more achievable in seeing you go through the difficulty first. Thank you all. I have been able to do so much more through your love and support.

Thank you to all who have helped me in my thesis, my college experience, and life. 


\section{TABLE OF CONTENTS}

LIST OF FIGURES X xiii

LIST OF EQUATIONS Xvi

CHAPTER 1 - INTRODUCTION

1.1 Motivation 1

1.2 Epidermis 4

$\begin{array}{ll}1.3 \text { Cancer } & 6\end{array}$

1.3.1 Metastasis, and Risk Factors 6

1.3.2 Methods of Skin Cancer Detection 8

$\begin{array}{lr}\text { 1.3.3 Cancer Markers } & 10\end{array}$

1.4 Quantum Dots 11

1.4.1 Molecular Orbitals and Band Theory 12

1.4.2 Quantum Confinement and Bohr Exciton Radius 13

$\begin{array}{ll}\text { 1.4.3 Fluorescence } & 14\end{array}$

$\begin{array}{ll}\text { 1.4.4 Potential as a Biomarker } & 15\end{array}$

1.4.5 Problems to Overcome with Quantum Dots as Biomarkers $\quad 16$

$\begin{array}{ll}1.5 \text { Phospholipids } & 18\end{array}$

1.6 Thesis Goal $\quad 20$

CHAPTER 2 - DEVELOPMENT AND EVALUATION OF CdSe/ZnS

$\begin{array}{ll}\text { QUANTUM DOTS } & 23\end{array}$

2.1 Introduction $\quad 23$

2.2 Quantum Dot Growth 25

2.3 Shelling Quantum Dot Cores 26 
$\begin{array}{ll}2.4 \text { Process Design } & 27\end{array}$

$\begin{array}{ll}2.5 \text { Sample Preparation } & 27\end{array}$

$\begin{array}{ll}2.6 \text { Testing Samples } & 29\end{array}$

2.6.1 Results of Initial Change from Quantum Dot Unshelled to Shelled State

2.6.2 Results of Chance in Quantum Dot Fluorescence over 4 Days

2.7 Analysis of Fluorescence Changes

2.7.1 Analysis of Initial Change in Unshelled to Shelled State

2.7.2 Change Over 4 Days: Shelled

2.7.3 Change over 4 Days: Unshelled Cores

2.7.4 Extractions at 0 Seconds

2.9 Additional Recommendations

CHAPTER 3 - SYNTHESIS OF QUANTUM DOT CONSTRUCTS

3.1 Introduction

3.2 Preparation for Encapsulation

3.2.1 Quantum Dot Precipitation Method

3.2.2 Quantum Dot Suspension into $\mathrm{CHCl}_{3}$

3.3 Calculations Required for Phospholipid Encapsulation Process

3.3.1 Quantum Dot Size

3.3.2 Quantum Dot Quantity 
3.3.4 Phospholipids per Square Nanometer of Quantum Dot

Surface Calculation

3.4 Phospholipid Encapsulation Process

3.4.1 Weighing and Addition of Phospholipids

3.4.2 Rotary Evaporation

3.4.3 Resuspension in Water and Buffer

3.5 Experimental Procedure Variations for Quantum Dot Encapsulation

52

3.5.1 The Two Sample Extraction Types

3.5.2 Altering the Concentrations of DPPC

3.6 Fluorescence Characterization

3.7 Results

3.7.1 DSPC vs DDPPC Fluorescence

3.7.2 The Altered Concentrations of DPPC

4.1 Chloroform/ODE Suspension vs Aqueous Quantum Dots

4.2 Full Samples vs Partial Samples 66

4.3 The Superiority of DPPC Phosphocholine Phospholipids 67

4.4 The Ten Phospholipids per Square Nanometer of Quantum Dot Surface Concentration

4.5 Three-DPPC per Quantum Dot Surface Fluorescence

4.6 Areas for Improvement within Current Methods 
APPENDIX A1: "SMALL BATCH" CdSe SYNTHESIS PROCEDURE WITH SILICONE OIL BATH FOR HEATING

APPENDIX A2: ZnS COATING PROCEDURE

APPENDIX B -STATISTICAL ANALYSIS OF UNSHELLED TO

SHELLED QUANTUM DOTS 


\section{LIST OF FIGURES}

Figure 1 A cross sectional view of the layers of the epidermis. 5

$\begin{array}{lll}\text { Figure } 2 & \text { The process of metastasis. } & 7\end{array}$

$\begin{array}{lll}\text { Figure } 3 \text { Various types of biopsies. } & 9\end{array}$

Figure 4 The fluorescent wavelength of QDs vary in size. 11

Figure 5 Quantum dots hold characteristics of both atomic particles and bulk substances.

Figure 6 As dimensions are confined, energy states become increasingly constrained.

Figure 7 Electrons are excited by UV photons.

Figure 8 A quantum dot surrounded by surfactant chains to prevent coalescence of the particles.

Figure 9 A standard phospholipid has a polar head composed of a polar group, a phosphate and (commonly) glycerol.

Figure 10 The surfactant chains can bind with an external chain source such as a phospholipid to encapsulate the particle.

Figure 11 A model of a CdSe QD core exhibiting the crystalline nature of the particle.

Figure 12 A model of a CdSe QD core shelled in ZnS.

Figure 13 Visual model of QD core synthesis process.

Figure 14 Incident light from a UV source at $385 \mathrm{~nm}$ wavelength is directed onto QDs. 
Figure 15 A representation of the full width at half max is indicated by the dashed line at the green arrow.

Figure 16 A schematic of a typical rotary evaporation machine.

Figure 17 Two variations of phosphocholine phospholipid.

Figure 18 Full sample measurement in the UV excitation spectrum of QDs processed with DSPC and DPPC phosphocholine phospholipids compared to a QD control group processed with no phospholipids.

Figure 19 Full sample measurement in the QD Fluorescent spectrum of QDs processed with DSPC and DPPC phosphocholine phospholipids compared to a QD control group processed with no phospholipids. A poor signal to noise ratio is seen.

Figure 20 Partial sample measurement in the QD fluorescence spectrum of QDs processed with DSPC and DPPC phosphocholine phospholipids compared to a QD control group processed with no phospholipids.

Figure 21 Partial sample measurement in the UV excitation spectrum of QDs processed with DSPC and DPPC phosphocholine phospholipids compared to a QD control group processed with no phospholipids. 
Figure 22 Full sample measurement in the UV excitation spectrum of QDs processed with various concentrations of DPPC phosphocholine phospholipids (per nm square) compared to a DPPC control group processed with no QDs.

Figure 23 Full sample measurement in the QD fluorescence spectrum of QDs processed with various concentrations of DPPC phosphocholine phospholipids (per nm square) compared to a DPPC control group processed with no QDs.

Figure 24 Partial sample measurement in the QD fluorescence spectrum of QDs processed with various concentrations of DPPC phosphocholine phospholipids (per nm square) compared to a DPPC control group processed with no QDs.

Figure 25 The partial sample values of the UV fluorescence spectrum for altering concentrations of DPPC with QDS compared to a pure DPPC control.

Figure 26 The longer tail chains of DSPC (left) allow for more movement of the polar head of the phospholipid. 


\section{LIST OF EQUATIONS}

Equation 1. Diameter $=\left(1.61222 \mathrm{E}^{-9}\right) \lambda^{4}-\left(2.6575 \mathrm{E}^{-6}\right) \lambda^{3}+\left(1.6242 \mathrm{E}^{-3}\right) \lambda^{2}-$

$$
\left(4.277 \mathrm{E}^{-1}\right) \lambda+(41.57)
$$

Equation 2. Surface Area $=4 \pi r^{2}$

Equation $3 . \quad$ Volume $=\frac{4}{3} \pi r^{3}$

Equation 4. Weight of Phospholipids to Add= Total Surface Area of QDs in Sample $\times$

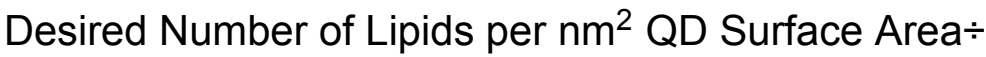
$\mathrm{N}_{\mathrm{A}} \times$ Molecular Weight of Phospholipid 


\section{Chapter 1 - Introduction}

\subsection{Motivation}

Cancer is the second leading cause of death in the United States, exceeded only by heart disease (1). Cancer rates are on the rise in the United States. According to the 2014 cancer statistics conducted by the American Cancer Society, it is predicted that for the United States alone 1,665,540 new cases of cancer will be diagnosed in 2014 . It is also expected that 585,720 of those in the United States who are presently living with cancer will die because of the condition over the same year (2).Cancer can grow rapidly if left unchecked, and can metastasize to other areas of the body (3). Cancer diagnosed at later stages has an adverse effect on survival rate. If detection is delayed until later stages of development, it is often too late for the patient to survive (4). One especially common form of cancer in the United States is skin cancer. In 2014, it is estimated that 76,100 people in the United States will be diagnosed with melanoma alone, and nearly 10,000 will die from it. That does not include other types of skin cancer such as basal and squamous cell carcinoma (2).

Early and accurate detection is the most reliable way to ensure a positive outcome and the survival of a person living with cancer. By the time some symptoms appear, the cancer may have grown and spread to a point where it is too difficult to cure (3). As melanoma is the most aggressive type of skin cancer it is particularly important to detect early (5). Current methods for initially detecting cancer rely largely on visual examination, palpitation, and for some of the most prevalent forms of cancer, routine screenings (3). If an area of tissue is 
assessed to be at risk for having cancer, a biopsy may be performed by a pathologist to confirm the presence of cancerous cells (6). Because this assessment dictates a treatment plan for the patient, it is critical that the results of the biopsy are accurate. In early stages of cancer development, detection may prove especially difficult, and though a patient may have cancer, the testing may miss the cancerous tissue. This leads to what is known as a false negative, where the test states there is no problem, when in fact cancer exists. This is likely to cause a patient to delay seeking additional medical care even if symptoms become worse (3).

To reduce false negatives and encourage the earliest possible treatment for cancer, it is important to design an alternative to the conventional methods of detection, namely biopsies. A possible improvement would be to utilize functionalized fluorescent nanocrystalline clusters of atoms known as quantum dots (QDs) constructs. These constructs could provide a means to detect even trace amounts of cancer that would likely be overlooked in a biopsy sample.

QDs are naturally hydrophobic, but can be functionalized to work within an aqueous environment by utilizing amphipathic compounds such as phospholipids. By attaching the nonpolar chains of QDs to the nonpolar chains of the phospholipids, the QD can be surrounded by the polar heads of phospholipids. This can allow the QDs to dissolve into a protic solvent such as water (7). QDs are being utilized as replacements for organic fluorophores in some biologic applications to target specified enzymes (8). Similar mechanics could be applied to target enzymes that are associated with the initial stages of 
cancer growth that are specific to cancerous cells in order to detect early stages of cancer. If cancer could be detected before metastization, patient survival would increase. Recently, many attempts have been conducted to target cancer cells with QDs.

The purpose of this thesis is to develop a standard for the aqueous suspension and functionalization of QDs utilizing phospholipids. The development procedure in this thesis aimed to design constructs which could be further processed to be utilized as a biomarker specifically for skin cancer. Quantum dots markers could potentially be observed in the layers of the skin without surgical intervention. Along with this, melanoma has been shown to have false negative diagnoses. This method has the potential to replace the subjective process of biopsies with a less subjective method for earlier detection of skin cancer.

This introduction presents an overview of the physiology and stages of development for cancerous cells, specifically melanoma. Along with this, ultraviolet (UV) radiation, epidermal tissue physiology, and the effects of UV radiation on epidermal tissue will be covered. In conjunction, the chemical properties and biological interactions of phospholipids, and quantum behavior of QDs will be discussed. In knowing the chemical and biological interactions of the phospholipids, the QD construct development will have greater clarity in its purpose. There will also be a brief summary of some of the alternative uses for QDs in biological systems to expand upon the diverse potential applications of QDs for medical purposes. 


\subsection{Epidermis}

To understand the cancer which the QD constructs have the potential to target, the cancer location must first be understood. The skin covers the surface of the human body and accounts for roughly $7 \%$ of the total body weight in the average adult. The skin serves to regulate body temperature, retain water within the body, and to protect the body from outside pathogens and physical harm. It has been isolated into two major layers, the vascularized deep tissue of the dermis, and the outermost nonvascularized layer known as the epidermis (9). Each can be divided into further sublayers. Skin cancer is most commonly known to originate in cells of the epidermis.

The epidermis is a keratinized stratified squamous epithelium consisting of four distinct cell types and four or five distinct layers. These cells are dendritic cells, tactile, keratinocytes, and melanocytes. Dendritic cells, also called Langerhans cells, ingest foreign substances, and are key activators of the immune system. Tactile cells, also called Merkel cells, are the sensory receptors for touch. Keratinocytes produce the fibrous protein known as keratin. Melanocytes are the cells which synthesize the pigment melanin. Melanin which act as a shield to protect from the damaging effects of UV radiation.

The layers of the epidermis, as seen in Figure 1, (ordered from outermost to deepest) are the stratum corneum, stratum lucidum, stratum granulosum, stratum spinosum, and stratum basale. The stratum corneum is a mostly superficial layer of dead cells. The stratum lucidum is only found in areas subject to abrasion such as the palms and soles of feet, and acts as an additional 
protective layer. The stratum granulosum is the layer in which organelles begin to deteriorate and fill with keratin. The stratum spinosum where cells are held in a web-like system, and pre-keratin is formed. The deepest layer of the epidermis is the stratum basale. In the stratum basale, a single row of stem cells continually renew cell population, producing a variety of keratinocytes, including melanocytes.

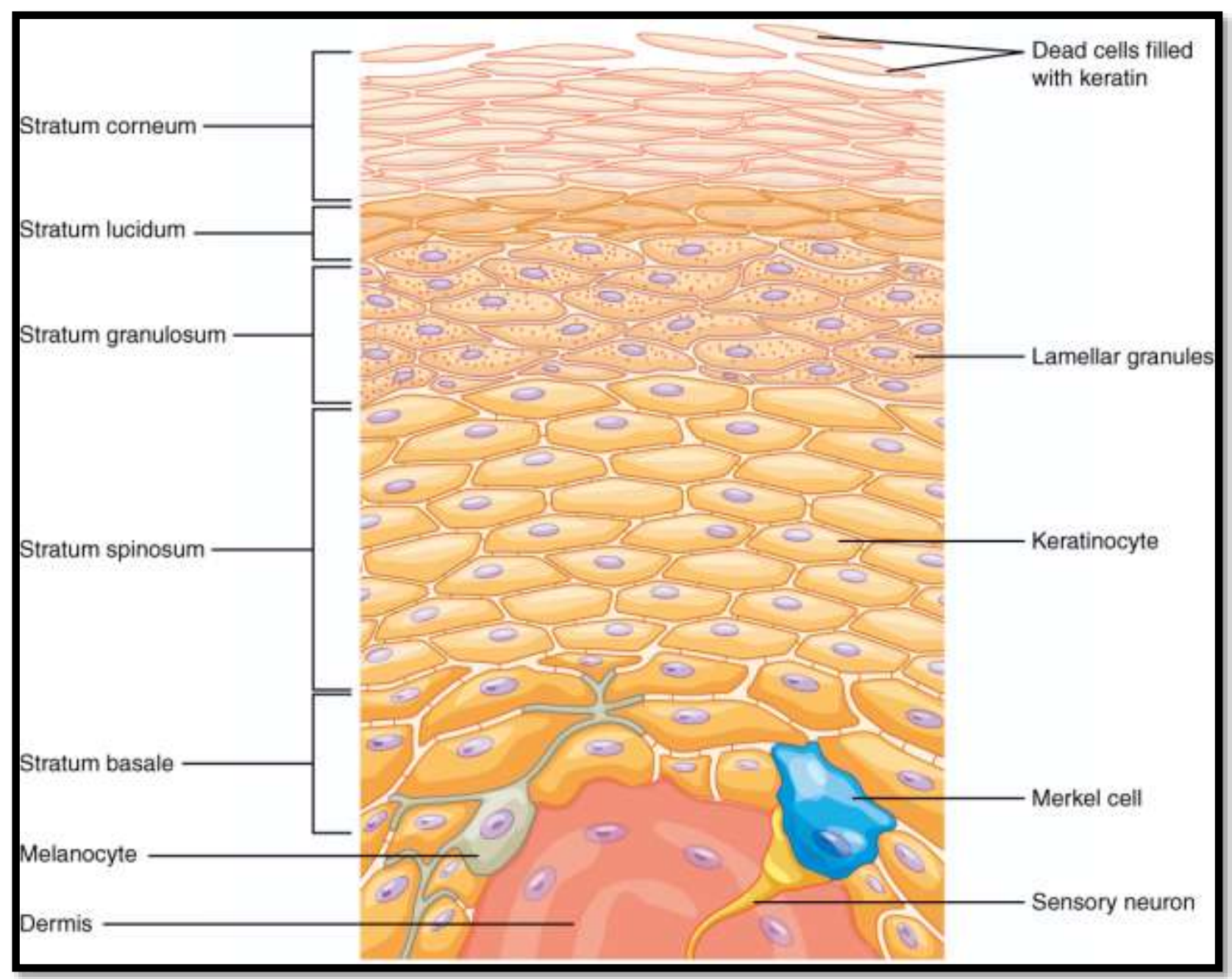

Figure 1: A cross sectional view of the layers of the epidermis. (10)

Depending on which skin cells mutate, and in which layer the mutation occurs different forms of cancer occur. If mutated keratinocytes form in the 
stratum basale, it is may become basal cell carcinoma. If it is the keratinocytes of the stratum spinosum that are mutating, it may become squamous cell carcinoma. If the melanocytes are mutating, melanoma may occur (9). While cancers are often caused by mutation, a mutated cell is not necessarily cancerous.

\subsection{Cancer}

Cancer refers to a wide variety of diseases characterized by the development of abnormal cells that divide uncontrollably and may infiltrate and destroy normal healthy tissue. Cancer has the ability to spread through the body (11). When a cell fails to follow normal controls of cell division, and multiply to excess, an abnormal mass of proliferating cells known as a neoplasm forms. Neoplasms may be either benign or malignant. A benign neoplasm retains itself in a localized area, tend to grow slowly, and seldom kill their host if removed before they compress vital organs. In contrast cancers are malignant neoplasms. Cancer cells invade their surroundings and may travel via blood or lymph to other body organs where they form secondary cancer masses. This traveling capability is known as metastasis (9).

\subsubsection{Metastasis, and Risk Factors}

Metastasis follows a series of stages as depicted in Figure 2. Cells grow as a tumor. They then break through the basement membrane. This is followed by an invasion of surrounding tissue. If the cells reach the lymphatic system or 
blood vessels, they travel through the body. Some cells adhere themselves in another portion of the body. At this point they spread through the wall and proliferate to start forming a secondary tumor (12).

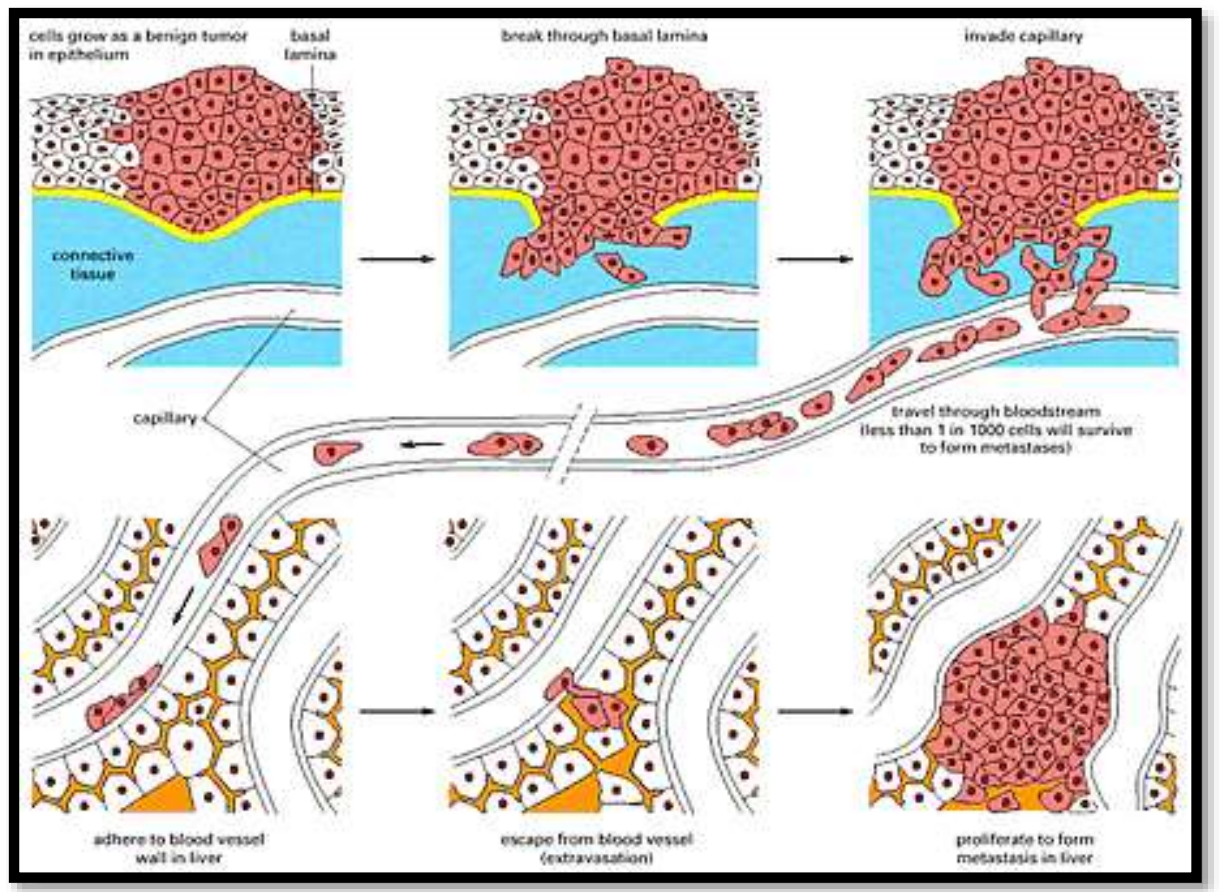

Figure 2: The process of metastasis. An initial mass of cancer spreads from the starting location until reaching a body's transport system. The cancer spreads through this system starting a new tumor in a different location.(12)

There are a wide variety of factors that put individuals at risk for cancer. Some families have a genetically heightened risk for cancer. This can be in direct hereditary mutations that are passed from a parent to a child, or genetic mutations that are acquired later in life (13). Roughly one in 10 patients diagnosed with melanoma have a family member with a history of the disease. Each person with a first-degree relative diagnosed with melanoma has a 50 percent greater chance of developing the disease than people who do not have a family history of the disease. Individuals belonging to a melanoma family with Familial Atypical Multiple Mole Melanoma Syndrome (FAMMM) have a 
heightened risk of melanoma (14). Some genetic traits even increase cancer risk indirectly. Pale skin is known to increase the risk of developing skin cancer (5).

Along with genetic influences, the mutations which develop later in life are often caused by environmental factors such as exposure to radiation or toxins. The single most important risk factor for skin cancer is overexposure to UV radiation, which damages DNA bases (9). UV radiation can come from the sun, or man-made sources such as tanning beds and welding torches (15). UV radiation appears to damage DNA bases, and disable tumor suppressor genes (9). If normal genes known as proto-oncogens (genes that control how often a cell divides) mutates, it can become a cancer-causing gene known as an oncogene (13). Tumor suppressor genes slow cell division, repair DNA mistakes, and cause apoptosis (programmed cell death). When these genes do not function properly, cells may grow out of control leading to cancer. (16).

\subsubsection{Methods of Skin Cancer Detection}

There are methods in place to determine if an individual has skin cancer. The most prevalent way to determine if a patient has skin cancer is to analyze a skin lesion (abnormal area of skin) biopsy. A skin lesion biopsy is the removal of a piece of suspected abnormal skin to diagnose or rule out an illness such as cancer (17). There are several ways to perform a skin biopsy. There are shave biopsies, punch biopsies, excisional biopsies, and incisional biopsies, as seen in 
Figure 3. The type of biopsy selected depends on the size, location, and type of lesion. An excisional biopsy is usually performed by a surgeon. The entire lesion is removed, including beneath the skin. An incisional biopsy, also commonly performed by surgeons, removes a large piece of the lesion for examination. A shave biopsy is the least invasive method, where a small blade such as a razor is used to remove the outermost layers of the skin. A punch biopsy may be used for deeper lesions. A specialized skin punch tool is used to remove a small round piece of skin (17). Once the biopsies are removed from the patient, the specimen is placed in a preservative fluid and sent to a pathology lab. At the laboratory, a pathologist, dermatologist,

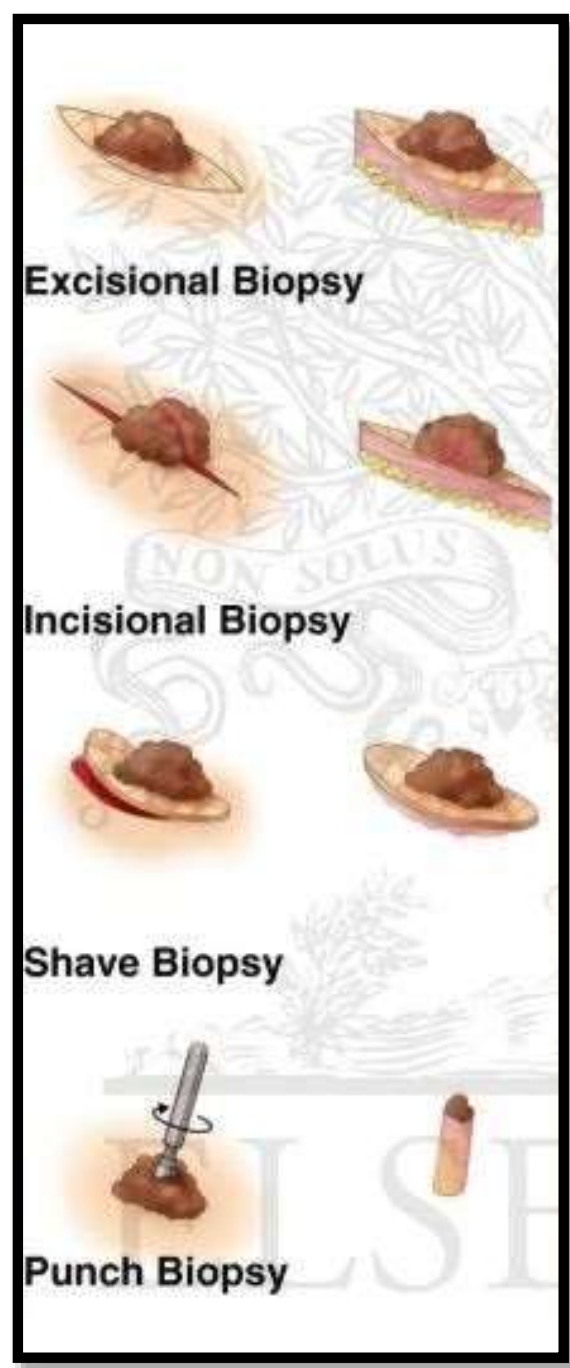

Figure 3: Various types of biopsies. (18) or an assistant may first observe the specimen without a microscope first in a gross examination (meaning seen without a microscope). Thereafter, or if the specimen is too small, the specimen prepared for histological observation. The cells are then visually observed for signs of abnormality that may indicate cancer. Abnormal cell size or shape are indicators of cancer. If a cell's nucleus is of abnormal size or shape, it may be indicative of cancer as well. Malformed or distorted glandular cells, or cell groups are also good indications that cancer is 
present. If certain types of cells found only in specific areas of the body are found in an unrelated area, it is likely that cancer has spread this tissue type to the new location through metastasis (19). The most common error with a biopsy is a false negative, which occurs when a medical professional fails to detect cancer when a patient does have cancer (20). Some studies have seen false negatives in as many as $16 \%$ of patients (21). A false negative can cause a patient to ignore dangerous cancer progression for prolonged periods of time, allowing the cancer to spread. As cancer progresses undiagnosed and untreated, patients are more likely to die because of the cancer.

\subsubsection{Cancer Markers}

Cancer cells are often associated with unique markers. These markers can be created by the cancer, or by the body in response to cancer. Most markers are proteins, but some are genes or other substances. The markers can be found in bodily fluids such as blood or urine, or inside of and on the tumors. While presently tumor markers are utilized to monitor the condition of those already diagnosed with cancer, they may hold greater potential. Many markers are specific only to one or a few types of cancer. One example of this kind of marker is a mutated $B R A F$ gene. The mutated $B R A F$ gene is an altered protein that causes melanoma cells to grow and divide abnormally. It is found in about half of melanomas (22). If a biomarker can be made to attach to a specific cancer marker, then cancer markers could be utilized as part of an early detection system. Biomarkers gathering in a specific area of the body could indicate a 
concentration of cancer markers on a tumor in the earliest stages of growth. It is possible to attach these markers to quantum dots using phospholipids.

\subsection{Quantum Dots}

QDs are being designed to act as biomarkers for early, reliable, non-subjective cancer detection. QDs are semiconductor nanocrystals that are typically composed of a hundred to a thousand atoms (a cluster is $2-10 \mathrm{~nm}$ in diameter). Because of their small size, QDs maintain some characteristics of bulk materials while also retaining characteristics of individual atoms. QDs exhibit properties of both classical and quantum physics. This unique pairing allows a direct influence over fluorescence characteristics by simply changing the size of the QDs, as seen in Figure 4 (23). The distinctive electronic and fluorescent properties of QDs can be explained by the high surface area to volume ratio as well as a property known as quantum confinement.

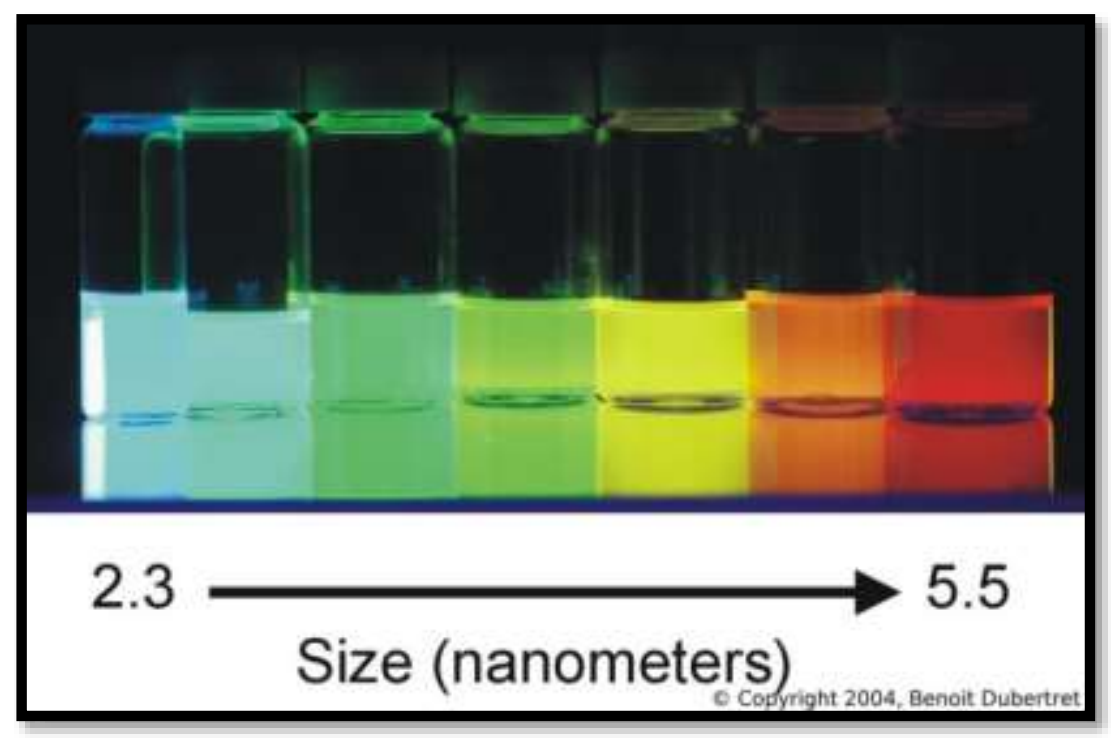

Figure 4: The fluorescent wavelength of QDs vary in size. Diameter sized are given in nanometers. As size increases, the fluorescent wavelength shifts from blue towards red.(23) 


\subsubsection{Molecular Orbitals and Band Theory}

Electrons exist in orbitals surrounding an atom's nucleus. The orbitals closest to the center of the nucleus have the lowest energy, while shells further from the nucleus have increasingly higher energies. Electrons have a strong tendency to occupy the lowest energy state possible, so long as no two orbitals on the same molecule have the same energy (24). This property also extends into bulk materials. As more atoms come into interaction with each other, orbital energies will shift to accommodate each other. Eventually, these shifts become so small that they can be regarded as continuous bands of energies. In bulk semiconductors, a valence band and a conduction band form. QDs are in a unique limbo between the atomic and bulk properties. They maintain discrete energy levels for electrons to occupy (as in atoms), while starting to act more like a bulk material as seen in Figure 5, and having a structure similar to bulk materials.

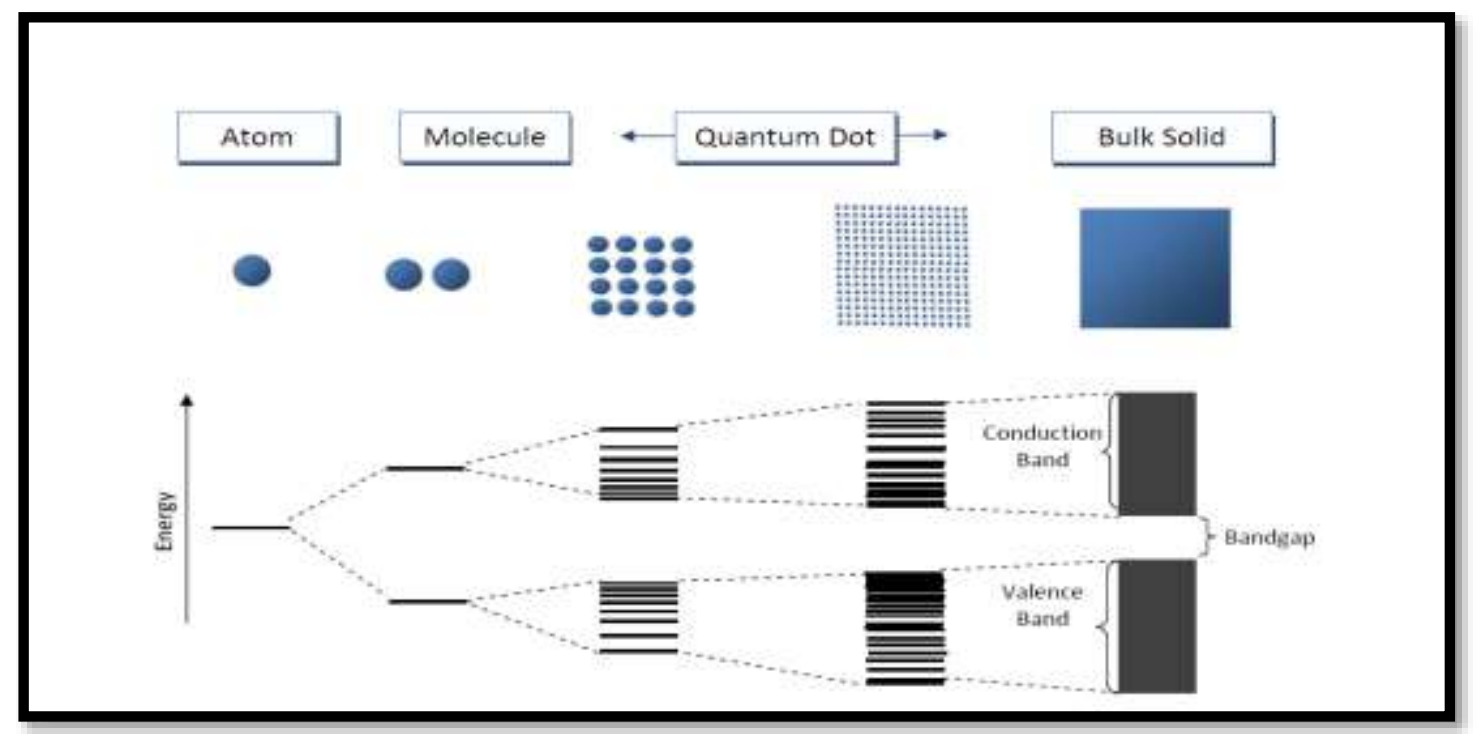

Figure 5: Quantum dots hold characteristics of both atomic particles and bulk substances. They have few discrete energy levels that exhibit band-like behavior. As size increases, more energy states become available. (25) 
The majority of electrons stay in the valence band, while few electrons move into the conduction band (26). When electrons are stimulated with enough energy (e.g. via the absorption of a photon), they may cross the distance between the valence and conduction band, known as the bandgap.

\subsubsection{Quantum Confinement and Bohr Exciton Radius}

When electrons jump from the lower energy levels to the higher energy levels, they leave behind a "hole". A hole is the positively charged area left behind when an electron transitions the bandgap from the valence to the conduction band. The pair of an electron and the hole are known as an exciton (27). The distance between an exciton's electron and its hole is known as the exciton Bohr radius. In a bulk material the exciton Bohr radius is miniscule in comparison to the material. As the material's size decreases to the nanoscale, as it does with QDs, the exciton Bohr radius is constricted. The electron and the hole are placed closer together than they would be in a bulk lattice, yielding higher electrostatic forces between the two (28). When the material is confined in one dimension, it creates a quantum well. Confinement in two dimensions creates a quantum wire. Total confinement yields a quantum dot. Each of these progressions alters the density of states in comparison to the energy as seen in 
Figure 6. The electron energy levels move from continuous to discrete energy states as they lose degrees of freedom.

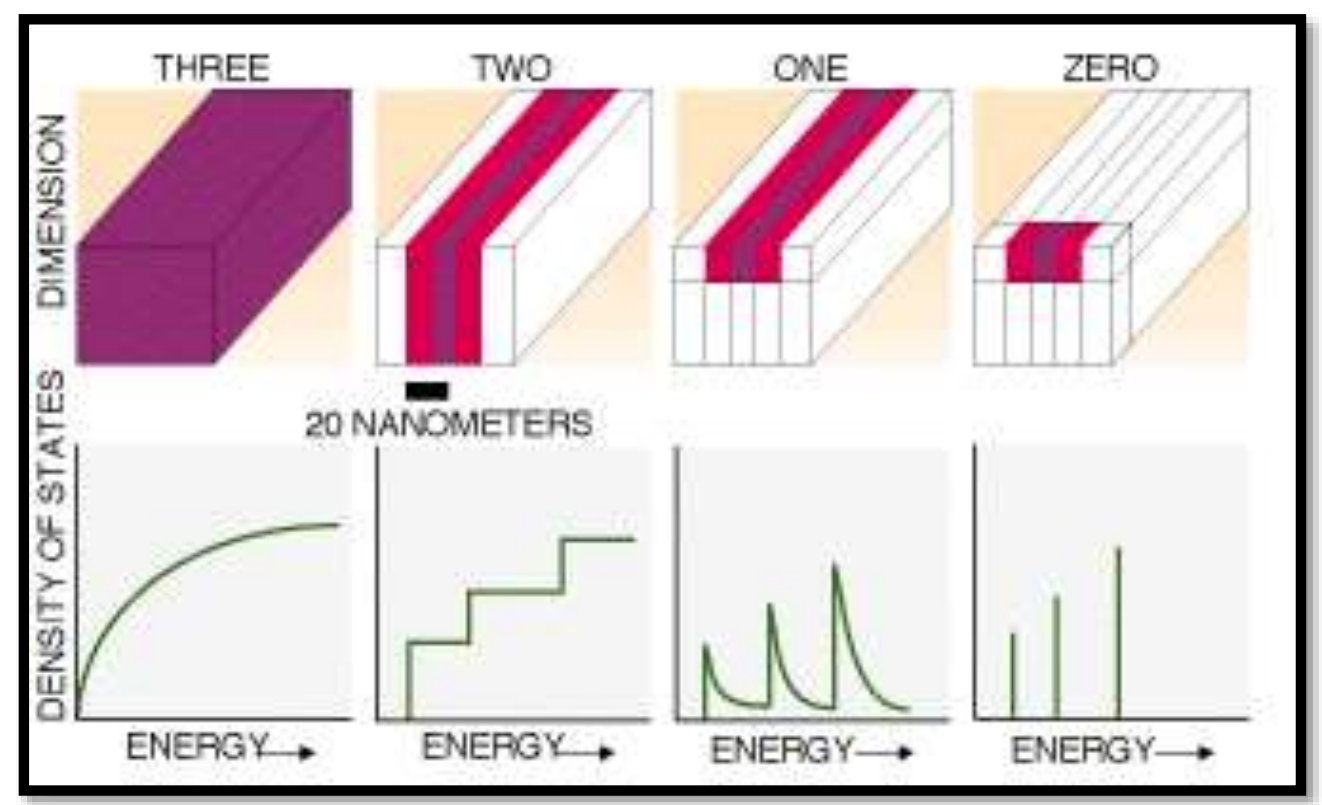

Figure 6: As dimensions are confined, energy states become increasingly constrained. (Left to right) A bulk material (0 dimension confinement), a quantum well (1 dimension of confinement), a quantum wire (2D confinement), and a quantum dot (3D confinement). (29)

\subsubsection{Fluorescence}

Once electrons are excited into the conduction band, they want to lose energy and transition to a lower energy state, as described with the Aufbau principle (24). When electrons relax to the ground state, the energy needs to leave the material in a new form. This can be achieved in a combination of nonradiative decay (loss of energy through heat) and radiative decay (loss of energy through photon emission) as seen in Figure 7 (30). While nonradiative relaxation occurs between energy states that are close to each other, radiative decay occurs most often in the final transition from the conduction band back down to the ground state of the valence band. Ultraviolet light is used to excite the QD electrons into excited states. The radiative relaxation is what causes the visible 
fluorescence of QDs. Smaller QDs will have a larger bandgap and produce light of a higher frequency or of a shorter wavelength (31).

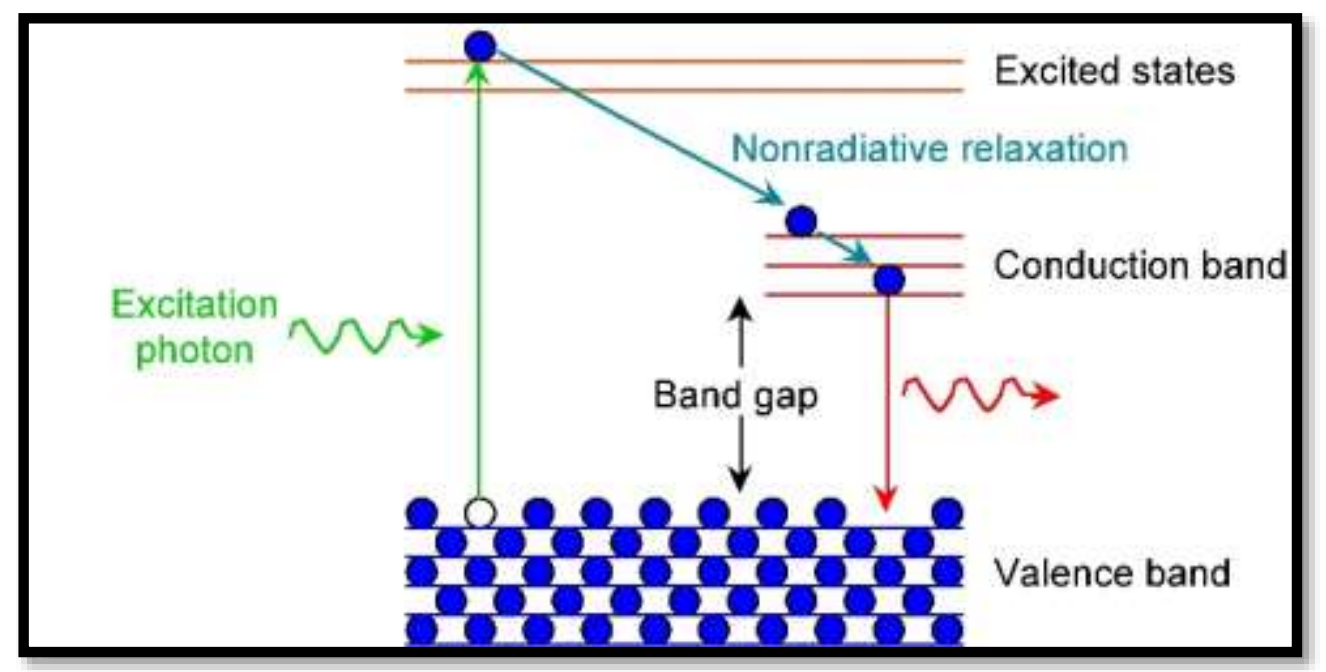

Figure 7: Electrons are excited by UV photons. They leave behind a hole. As the electron relaxes to the valence band from the excited state, it releases energy in the form of nonradiative and radiative decay. A photon is released. (30)

Fluorescence can be characterized several ways. Three main factors of fluorescence are important to potential QD biomarkers; their intensity, full width at half maximum (FWHM), and central wavelength. Intensity is a measurement of how many photons are being released. A brighter light will have a higher intensity. FWHM indicates the width of a waveform at half of the maximum intensity. A narrow FWHM in fluorescing QDs indicates similarly sized QDs. A narrower spectrum of fluoresced light would also be more distinct and easier to detect. Central wavelength indicates the most common wavelength of emitted photons from the QDs. It is a good way to predict what color QDs will fluoresce.

\subsubsection{Potential as a Biomarker}

QDs' distinctive fluorescence make them potentially useful as a biomaterial. They already have been used in a broad range of biological applications, including single molecule biophysics, optical barcoding, as well as 
molecular, cellular, and in vivo imaging. Compared to more common organic dyes and fluorescent proteins, QDs are about 10-100 times brighter. They are also more resistant to a phenomena known as photobleaching, the chemical breakdown of a dye or fluorophore (8). This means that not only do the QDs have the potential to be brighter than their organic dye and fluorophore counterparts, they are also more resistant to breakdown in the human body. Though they hold many advantages, the switch to the new technology will not be without caution. QDs have several hindrances to overcome, and unknown aspects to discover before they can be used safely within the human body.

\subsubsection{Problems to Overcome with Quantum Dots as Biomarkers}

The materials which compose many QDs are often harmful to the human body. The QDs in the experiments performed were created with a cadmiumselenium core. While selenium is required by the body for proper functionality in small amounts, in excess it is poisonous. Cadmium is a highly toxic heavy metal and is a known carcinogen (32). Whether for benefit or hindrance, QDs may behave differently than their bulk compounds.

One disadvantage of QDs is that they may pose a unique threat to the human body because of their small size. The small size of the particles may result in unusual toxicity compared to the bulk material, as well as providing new pathways for exposure where larger particles would not go, such as through a cell membrane (33). The particles are so small they may be able to be absorbed into tissues and cell walls. Chemical modifications to the surface play a large role in QD toxicity as well. Even if the surface is processed to minimize QD 
toxicity, it is important to consider the possible effects of metal release upon QD degradation. In vivo studies utilizing rodent models have indicated QDs may induce inflammation and oxidative stress in some bodily systems (QD Lung Inflammation). Studies have shown QDs elicit toxicity in a variety of in vitro systems. The same studies indicate that the toxicity of QDs can be minimized through selection of an appropriate shell coating, modulating surface charge, selecting a low overall dosage, and modulating the overall QD size (7).

Another disadvantage QDs have is that their size may lead to steric hindrance in some applications. Steric hindrance occurs when the large size of groups within a molecule prevent reactions that are observed in related molecules with smaller groups. QDs are larger than organic dyes that are currently used in many imaging applications. Due to the comparative size increase, the organic dyes may remain the superior option to QDs in some specific imaging applications (8).

Along with this, QDs themselves are often hydrophobic particles. This means QDs are resistant to being soluble in water. To prevent particle aggregation, QDs are often prepared as ligands, utilizing compounds such as trin-octylphosphine oxide (TOPO) and tri-octylphosphine (TOP) to surround the QD surface. The ligands act in such a way that the QDs do not aggregate, and become hydrophobic. In this state, QDs are only soluble in organic solvents (34). A representation of this can be seen in Figure 8. 


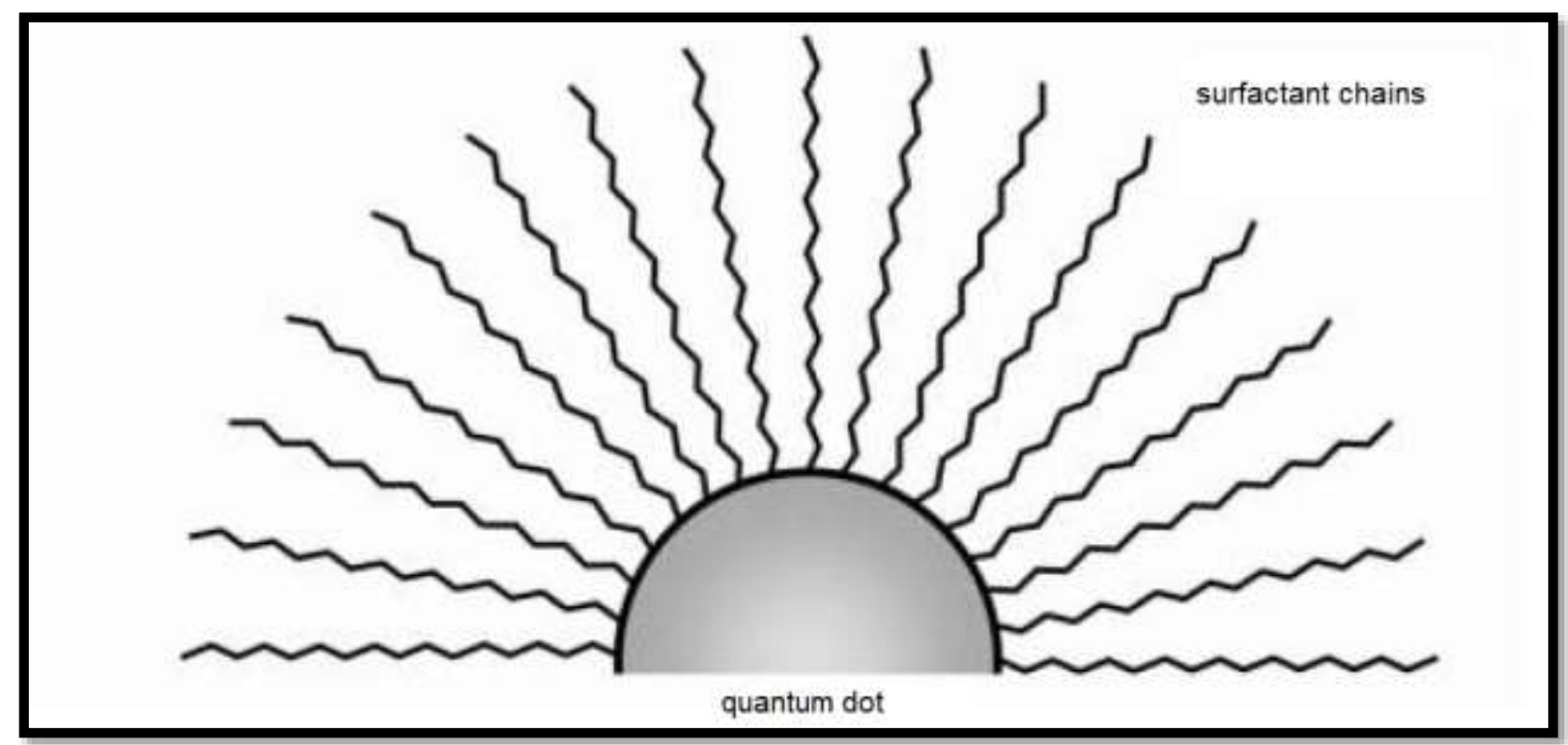

Figure 8: A quantum dot surrounded by surfactant chains to prevent coalescence of the particles.(34)

To functionalize the QDs for use as biomarkers, which can bind to hydrophilic compounds within the body, they need to be altered. A common method of doing this is to coat the surface of the QD with molecules that may attach to the surface of the QD, having a have a functional group on one end and a hydrophilic group on the other (35).

\subsection{Phospholipids}

To surmount some of the shortcomings of QDs, phospholipids may be useful. Phospholipids are a class of lipids. They can be found in the lipid bilayer of cell membranes, as well as in other natural compounds. Most phospholipids contain a diglyceride (a hydrophobic fatty acid chain which makes the "tails"), a phosphate group (part of the hydrophilic "head"), and a simple organic molecule such as choline (a polar component of the "head"). Though most phospholipids also have a glycerol backbone connecting the polar and nonpolar regions, 
Sphingomyelin is one exception to this, having sphingosine instead of glycerol. A visual model of a typical phospholipid can be seen in Figure 9.

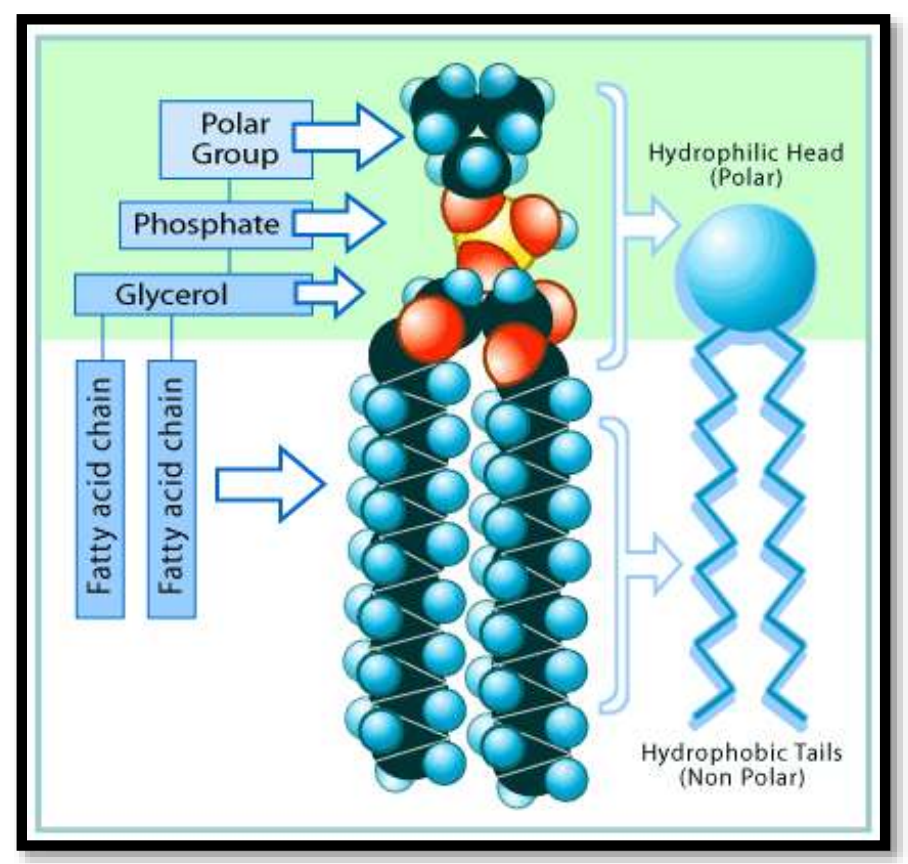

Figure 9: A standard phospholipid has a polar head composed of a polar group, a phosphate and (commonly) glycerol. The nonpolar tails attached to the glycerol are found in the form of fatty acid chains. (36)

Phospholipids exhibit amphipathic character. While the "head" of a phospholipid is hydrophilic, the "tails" of the molecule are hydrophobic. The hydrophilic head contains a negatively biased phosphate group and may contain other polar groups. The tail usually contains a long fatty acid hydrocarbon chain (37). By utilizing phospholipid's amphipathic character, the hydrophobic QDs can be converted from hydrophobic to hydrophilic. The hydrophobic chains of the QD have the potential to undergo interdigitation with the hydrophobic chains of the phospholipids. By binding the compounds surrounding the QD ligands (SUCH AS TOPO or TOP) to the phospholipid tails, the QDs become attached to the hydrophobic tails and surrounded by the hydrophilic heads of the phospholipids. 
This creates a functionalized hydrophilic QD conjugate as represented in Figure 10.

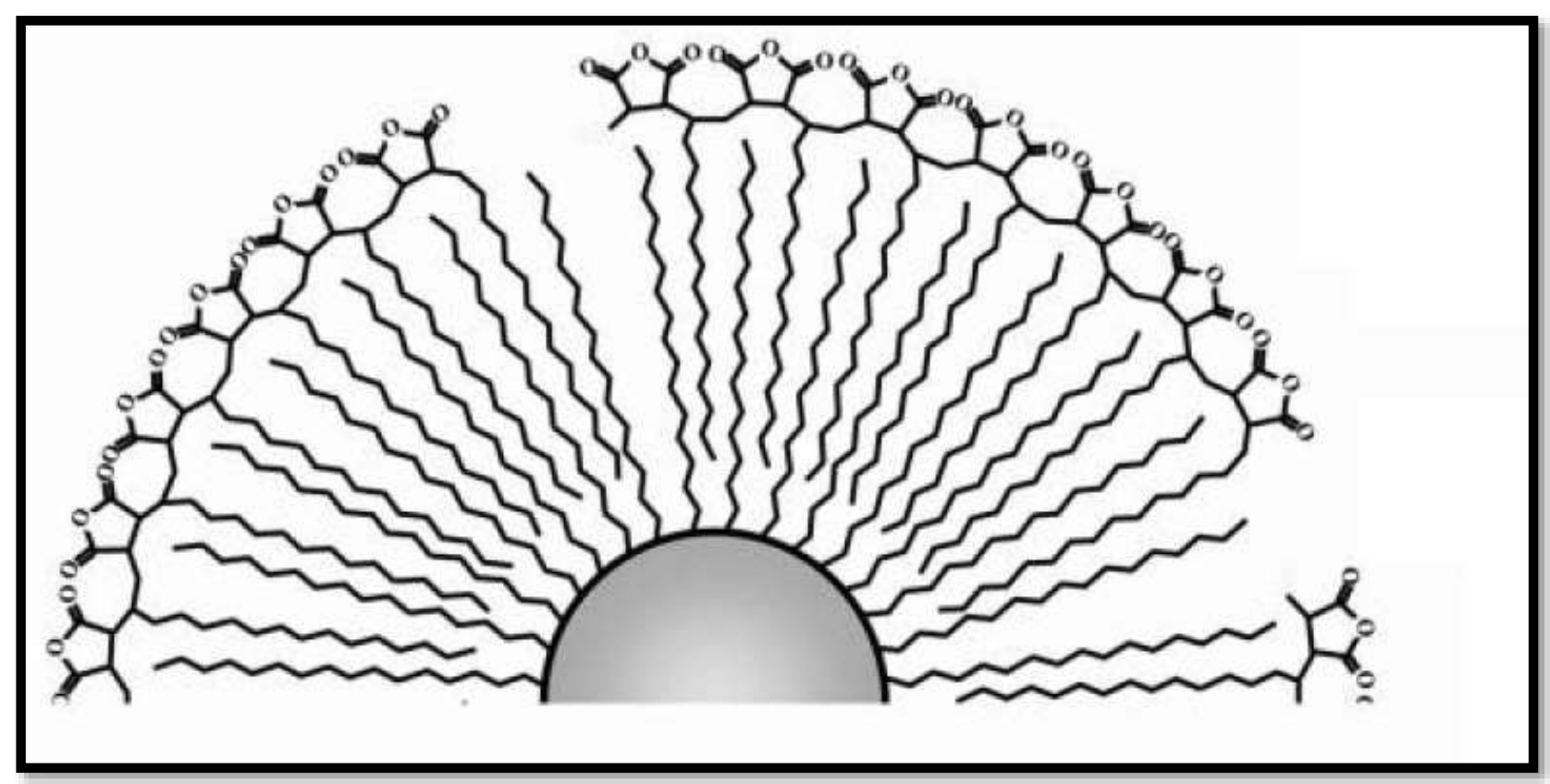

Figure 10: The surfactant chains can bind with an external chain source such as a phospholipid to encapsulate the particle. (34)

The two typed of phospholipids utilized in these experiments were 1,2distearoyl-sn-glycero-3-phosphocholine (DSPC) and 1,2-dipalmitoyl-sn-glycero3-phosphocholine (DPPC). Both are variants of phosphocholine. While the two molecules are highly similar, DSPC has a longer "tail" chain, and therefore has a higher molecular weight than DPPC (38).

\subsection{Thesis Goal}

This introductory chapter has been presented to provide the basic technical background relevant to this thesis. Detection and diagnosis of aggressive cancer in the earliest possible stage is critical for patient survival. 
The present methods of cancer detection, using biopsies analyzed by technicians and doctors, are inherently subjective, have great difficulty detecting small populations of abnormal cells, and may lead to false negatives. These false negatives can delay diagnosis and cancer treatment, allowing cancer to grow and spread further. This is directly detrimental to the chance of survival, especially in aggressive cancers. A less subjective method of detecting smaller amounts of cancer would greatly reduce false negatives. Functionalized QD constructs are one potential tool in accomplishing this task. QD constructs have the potential to identify trace amounts of cancer in an objective way by attaching to cancer cells and fluorescing at specific wavelengths for easy identification. They may have the potential to act as drug delivery devices as well.

The purpose of this thesis was to develop a foundational method for the solublization and initial functionalization of QDs for use as biomarkers. This was achieved through encapsulation of QDs of various emission wavelengths with a phospholipid coating. To accomplish this, CdSe QDs were synthesized utilizing an organometallic colloidal growth method developed at Cal Poly; this synthesis protocol uses variation in nucleation, temperature, and times to create QDs with the appropriate emission wavelengths. These QD cores were further coated in a ZnS shell to improve the fluorescent properties and stability of the QDs by reducing surface defects and broadening the potential spectrum of photons which can excite the QD. Fluorescence measurements of intensity, full width at half maximum (FWHM), and central wavelength were taken to observe the 
differences between unshelled QDs, shelled QDs, and QDs functionalized with phospholipids.

These shelled quantum dots were then run through an experimentation procedure, testing differing types of phospholipids at different concentrations. The alteration to these concentrations and phospholipid types was meant to discern a trend towards an ideal solubilization process. First two phospholipids were compared to distinguish which may have had the superior solubilization of QDs. The better of the two phospholipids was then tested at different concentrations in an attempt to discover a trend in how to further increase the solubility of the QDs.

The goal is to achieve a coating process for phospholipids without losing fluorescence intensity. This thesis describes the methodology for a solubilization and primary phospholipid functionalization of CdSe/ZnS QDs for further development as biomarkers for skin cancer detection and treatment. 


\section{Chapter 2 - Development and Evaluation of CdSe/ZnS Quantum Dots}

\subsection{Introduction}

While working for IBM in 1970, physicists Dr. Leo Esaki and Dr. Raphael Tsu were the first to produce crystalline superlattices via epitaxy of alternating ultrathin layers, which exhibited quantum mechanical effects (39). In the same year, IBM would be working with AT\&T Bell Labs to produce two-dimensional quantum wells. In 1983 these two-dimensional structures would further be refined at Bell Labs into single-dimensional QDs in 1983. The term "quantum dot" would not be coined until Mark Reed's first lithographic QDs in 1987 (40). This would not be the only way QDs were created.

The production of QDs can occur via multiple methods. These methods can be easily classified into the two groups of "bottom-up" and "top-down" synthesis. A top-down method starts with a macroscopic compound that is reduced down to appropriate dimensions through chemical and/or mechanical means. This process can be seen as similar to machining down a large block of metal into a smaller useful part. While effective, the process is often tedious and expensive (41).

The bottom-up synthesis can occur via multiple methods. These processes include epitaxial self-assembly, colloidal synthesis, and other methods (42). Epitaxial self-assembly begins with some form of atomic deposition onto a semiconductor substrate. The deposited material is selected to have a smaller bandgap than the substrate. Epitaxial "islands" spontaneously form for energetic 
reasons. These islands are made into QDs by covering them with another semiconductor layer, having a larger band gap than the islands (43). Colloidal synthesis does not rely on a substrate, but instead creates the QDs suspended and dispersed through a liquid by utilizing the nucleation and growth of the QDs in mixed precursors. These precursors contain the necessary elements (e.g. cadmium, sulfur, or selenium) to precipitate into QDs (41). In the organometallic precursor processes, chemicals (such as trioctylphosphane oxide) are often added to the process to passivate the QDs. This creates crystalline nanoparticles coated with surfactants which are highly monodisperse compared to other methods (41). Colloidal QDs may be created through molecular scaffold-based assembly, microbead-based assembly, arranging free QDs from a liquid solution, and even guided self-assembly using plasma lithography. The self-assembled or colloidal synthesized QDs are superior to the top-down approach because of their reduced synthesis time and reduced cost in materials. Additionally the resulting QDs are often smaller and have stronger quantum confinement potentials than the top-down counterparts (43).

A wide variety of elements have been utilized for the synthesis of QDs. The bottom-up methods commonly utilize elements from groups II - VI or III-V elements (e.g. CdSe, InP for II - VI and GaN for III - V). Both the bottom-up and top-down methods utilize group IV materials often utilizing, but not limited to silicon (41). Of the many combinations of elements used in QD synthesis, CdSe/ZnS core/shell QDs were among the first to be colloidally synthesized and retain a relatively high and stable luminescence yield. Since this process's 
development, there has been further research to replace the highly reactive metal organic precursors. In 2000, stable ionic precursors, or "green precursors" were introduced, greatly reducing some of the potential hazards encountered in synthesis. By making the process safer, this shift allowed a significant growth in the number of groups able to synthesize and investigate semiconductor nanocrystals. The years $2002-2007$ produced five times more scientific publications on colloidal QDs than the prior twenty years combined (44). This is evidence of the increasing interest in developing QDs for use in involved applications.

\subsection{Quantum Dot Growth}

QDs can be grown by multiple methods using multiple materials. This paper will focus on organometallic colloidal growth of CdSe QDs. In this process a precursor solution containing selenium (Se) is created and added into another precursor solution containing cadmium (Cd). Once the two precursors are mixed, Se and Cd ions begin to build up until they begin to overcome the energy barrier required for nucleation. This creates a multitude of small CdSe particles. After an initial burst of nucleation, the newly formed QDs will then begin to grow to larger and larger sized as the reaction continues. Extracting the QDs from this process at different points in time should yield different sizes of QD cores, ranging from approximately $2.7 \mathrm{~nm}$ to $4.6 \mathrm{~nm}$ in diameter. A representation of the QD core formed during this process can be seen in Figure 11. 


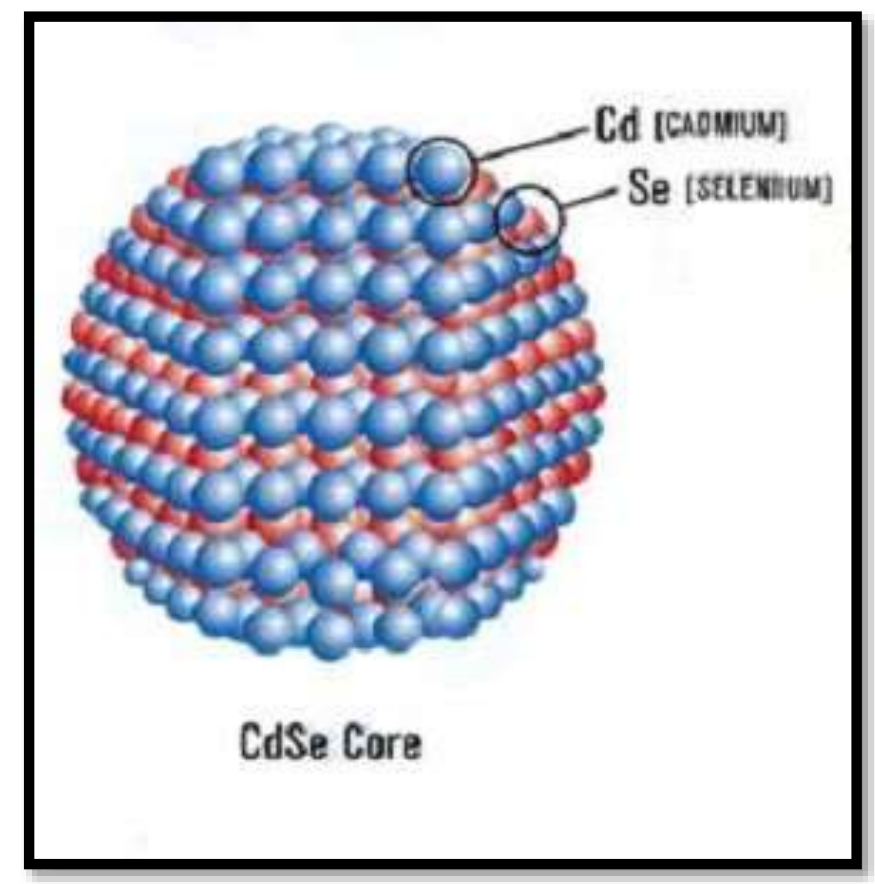

Figure 11: A model of a CdSe QD core exhibiting the crystalline nature of the particle.(45)

\subsection{Shelling Quantum Dot Cores}

After creating the QD cores, the cores can be shelled with another compound. This experiment used zinc sulfide for the shell. Because the zinc sulfide has a larger bandgap than the core of the QD, shelling the QD cores creates a "charge separation" in the QDs (46). The electrons want to stay in the core of the QD, but the holes want to go to the shell. This separation changes the properties of the QD. Of two electrons in the low energy state, one needs a significantly larger energy increase than the other, so it typically stays at the low level. As the other electron excites to the high-energy state and forms an exciton, it recombines in the presence of a photon and generates two photons to leave the material. Because of this, the fluorescence of shelled QDs should appear brighter than unshelled QDs, even when exposed to the same energy 
(46). A shelled QD encapsulates a QD core as modeled in Figure 12. A monolayer of $\mathrm{ZnS}$ is approximately $.31 \mathrm{~nm}(47)$.

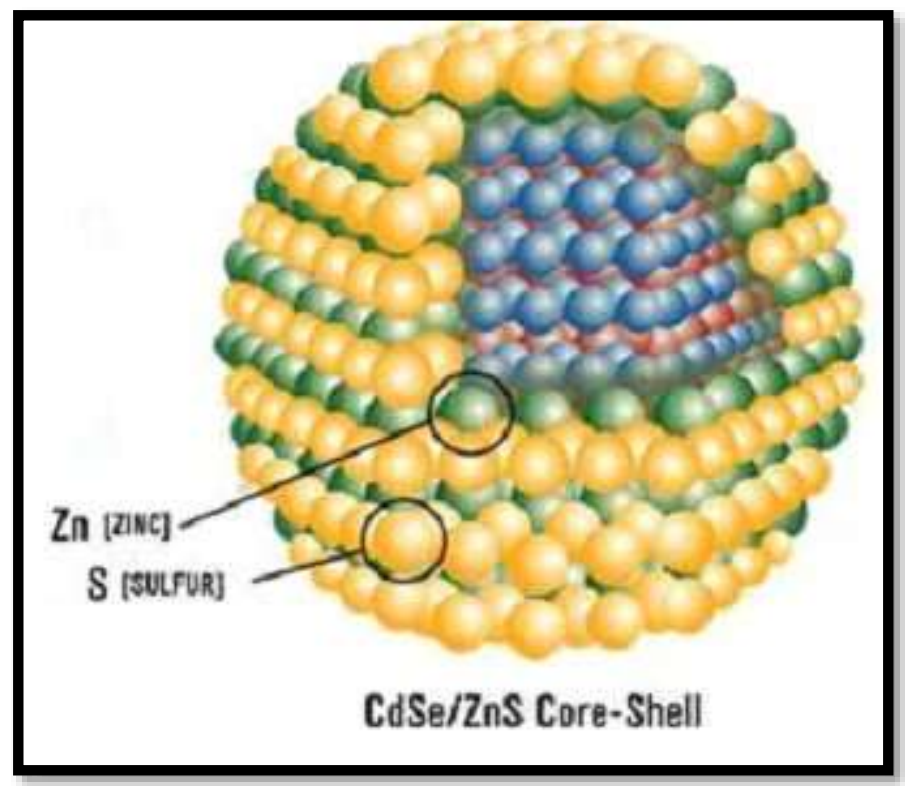

Figure 12: A model of a CdSe QD core shelled in ZnS. A cutaway is shown to display the CdSe core. (45)

\subsection{Process Design}

Goal of the experiment is to characterize the fluorescence of unshelled QDs over a four day span to their shelled counterparts. There was also a direct comparison of the QDs going from their unshelled state to the shelled state.

\subsection{Sample Preparation}

QD cores were synthesized utilizing an organometallic colloidal growth method. This process involves mixing two precursors, and allowing a chemical reaction to occur for a designated period of time before the solution (now containing QDs) is removed from the reaction vessel. Four extraction times were used in the experiment. Extractions were taken at 0 seconds, 30 seconds, 60 
seconds, and 90 seconds. This procedure yielded four vials unshelled QDs as seen in Figure 13.

\section{Synthesis Process}
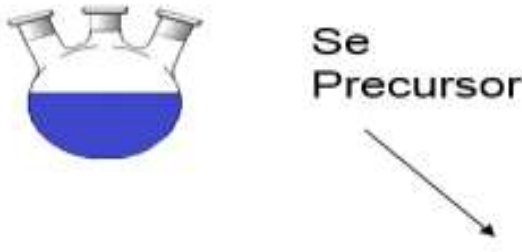

Seconds

Cd

Precursor

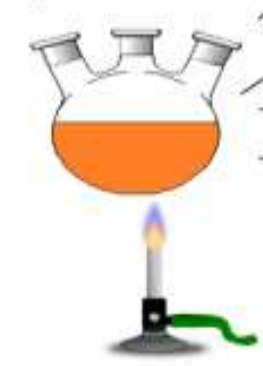

30 Seconds

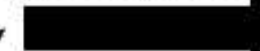

60 Seconds
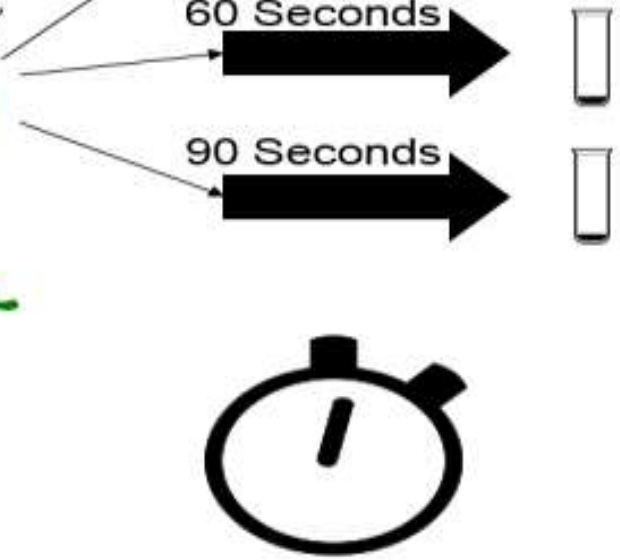

Figure 13: Visual model of QD core synthesis process. Selenium precursor is combined with cadmium precursor at 100 degrees Celcius. Samples were extracted at 0, 30,60, and 90 seconds.

At this point in the process, the unshelled vials were split into eight vials. Half of the QD solutions from each vial were set aside to remain unshelled for testing. The corresponding half of the extractions were then subjected to a shelling procedure. The unshelled QDs were mixed with a zinc sulfide solution to encapsulate the cadmium-selenium QDs in a zinc-sulfur shell. This process was performed at approximately $100^{\circ} \mathrm{C}$. Shelling QD samples needed to be done on an individual, sample by sample basis. The procedure for shelling one sample of QDs lasted 40 to 45 minutes on average, including the time required for the QDs to cool back to room temperature. This was done twice, to two separate batches 
of QDs, creating a total of 16 samples for analysis. There were eight unshelled samples and eight shelled samples.

\subsection{Testing Samples}

The area of interest in this testing was the fluorescence characteristics of the QDs. The fluorescence characteristics measured were the intensity, the full width at half maximum, and the central wavelength produced. The fluorescence characteristics of these 16 samples were analyzed in two main ways.

Fluorescence characteristics of corresponding pairs of unshelled and shelled QDs were compared (e.g. a 30-second-extraction of unshelled QDs from Batch A compared to their 30-second-extraction shelled QDs from Batch A counterparts). Along with the direct comparison of individual pairs, the unshelled and shelled QDs were compared as two groups to analyze how their fluorescence values changed over the course of four days. The fluorescence values of these samples were measured utilizing the testing setup shown in Figure 14. 


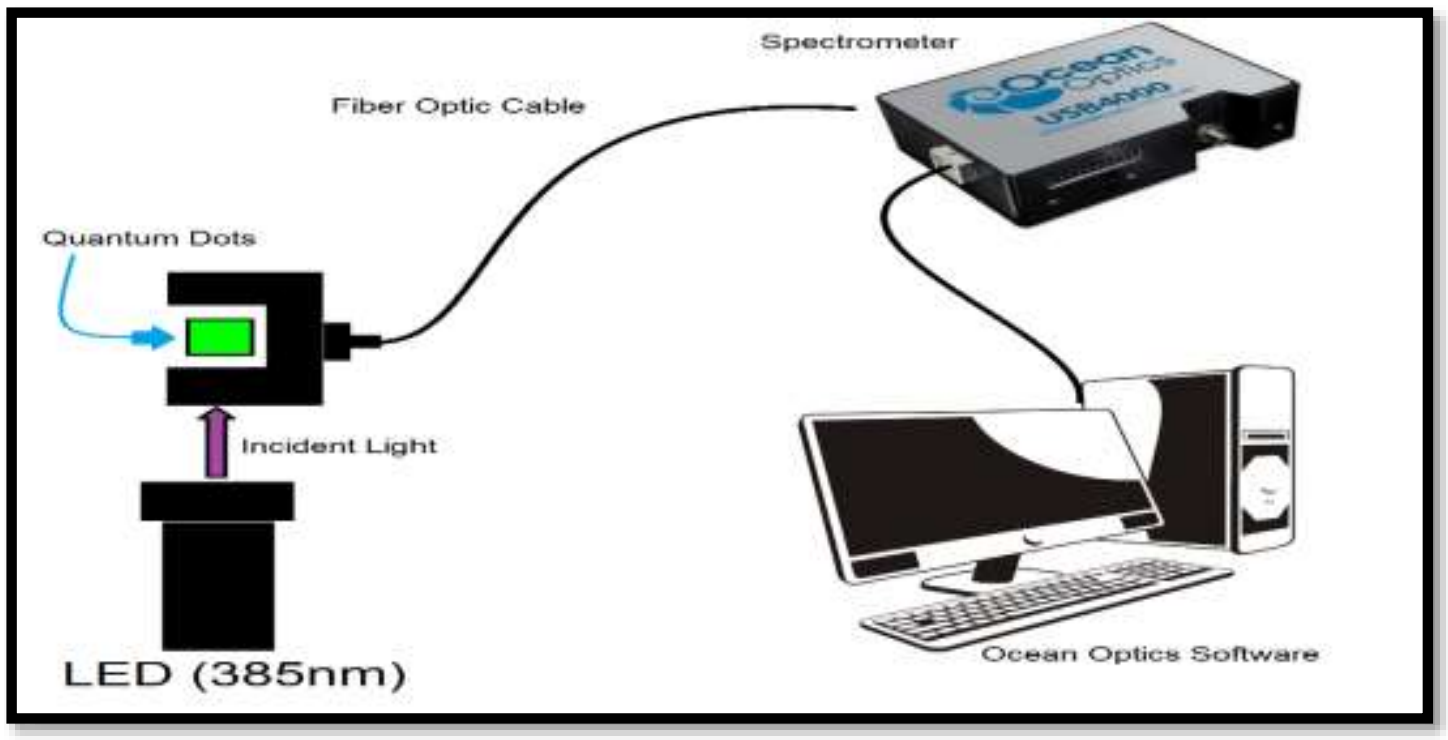

Figure 14: Incident light from a UV source at 385nm wavelength is directed onto QDs. A fiber optic cable at a right angle to the incident light relays the $Q D$ fluorescence to a spectrometer. The spectrometer sends this data to a computer for analysis. (48)

The QDs were loaded into disposable cuvettes and placed in a holding chamber. A minimum of $2 \mathrm{~mL}$ were used in each cuvette. In this chamber, they were exposed to an ultraviolet beam to make them fluoresce. This fluorescence was then conveyed down a fiber optic cable placed at $90^{\circ}$ the incident light, to an Ocean Optics spectrometer. This spectrometer relayed fluorescence values to a nearby computer, where the output was measured utilizing Spectra Suite software. In order to reduce experimental error, ten fluorescence measurements were taken per sample per day. This allowed for analysis of the QDs to be compared to a typical range of a day's measurements. This would later help indicate if changes in fluorescence values were statistically significant against the variances of a single test. Fluorescence data was taken on Day 0 (the day the QDs were shelled), Day 1 (the day after the QDs were shelled), and Day 3 (three days after the QDs were shelled). 
The resultant data was then stored in a Microsoft Excel workbook. This raw data was then processed into more useful and more easily analyzed dailyaverages. Along with the averages, standard deviations were generated so that the measurements between days could be observed to be similar to a single day's measurements or not.

The unshelled to shelled assessment compared the differences in the averages of individual unshelled samples and their shelled counterpart sample. These differences could then be averaged themselves, revealing the average shift of fluorescence characteristics when QDs transitioned from unshelled to shelled state.

A simultaneous study also monitored the unshelled QD fluorescence characteristics over the four days. Over the same timeframe, the shelled QD fluorescence values were monitored. This data set allowed for the stability of unshelled QD fluorescence to be compared to the stability of shelled QDs.

\subsubsection{Results of Initial Change from Quantum Dot Unshelled to Shelled State}

In going from their unshelled to the shelled state, QDs experienced an increase in counts of intensity. A "count" is a measurement of intensity utilized by Ocean Optics software. A greater intensity will have a higher count. The QDs saw an average increase in intensity of 3407 counts after being shelled. The population standard deviation of the unshelled and shelled intensity is roughly 71 counts. The observed increase is far outside the range of a day's error. Further calculations revealed this shift in intensity was a $278 \%$ increase from their unshelled state. Measurements were taken with an integration time of $5 \mathrm{~ms}$. A 
paired t-test was performed on the results, showing at least $95 \%$ confidence. The results of this can be seen in Appendix B.

The central wavelength also experienced shifts. Changes in central wavelength were not as simple as changes in intensity. While the central wavelength of 30 second, 60 second, and 90 second samples increased an average of $12.3 \mathrm{~nm}$, the 0 second samples experienced negative shifts in wavelength of $-81 \mathrm{~nm}$ and $-109 \mathrm{~nm}$. The population standard deviation was roughly $1.8 \mathrm{~nm}$. By excluding the more variable 0 second measurements, the population standard deviation drops even further to $1.2 \mathrm{~nm}$. This calculation is acceptable based on the fact that 0 second QD extractions were later decided to be excluded from future production. Though there were shifts in wavelength, they were not considered undesirable. This is because in application of a QD biomarker, the slight shift in wavelength should not have a drastic impact. As only the shelled QDs would be used, the average central wavelength would not be changing unpredictably between biomarkers. The shelled QD biomarkers still fluoresce in the spectrum of visible light.

There was also a shift in QD full width at half maximum (FWHM) going from the unshelled to a shelled state. Once again excluding outliers of the 0 second extractions, the FWHM had an average increase of $2.66 \mathrm{~nm}$. The population standard deviation for FWHM was only $0.52 \mathrm{~nm}$. This means that the average increase in FWHM, excluding the 0 second outliers, was $6.6 \%$. The 0 second FWHM were excluded because of the difficulty of using the software to get an accurate FWHM. As seen in Figure 15 the FWHM is best represented in 
measuring a single peak. The multiple peaks are most prominent in the 0 second values. While multiple peaks were seen in other extraction times, there was always a peak which overwhelmed the others in measurement. This allowed for the other FWHMs to be more representative of the actual FWHM of the dominant peak.

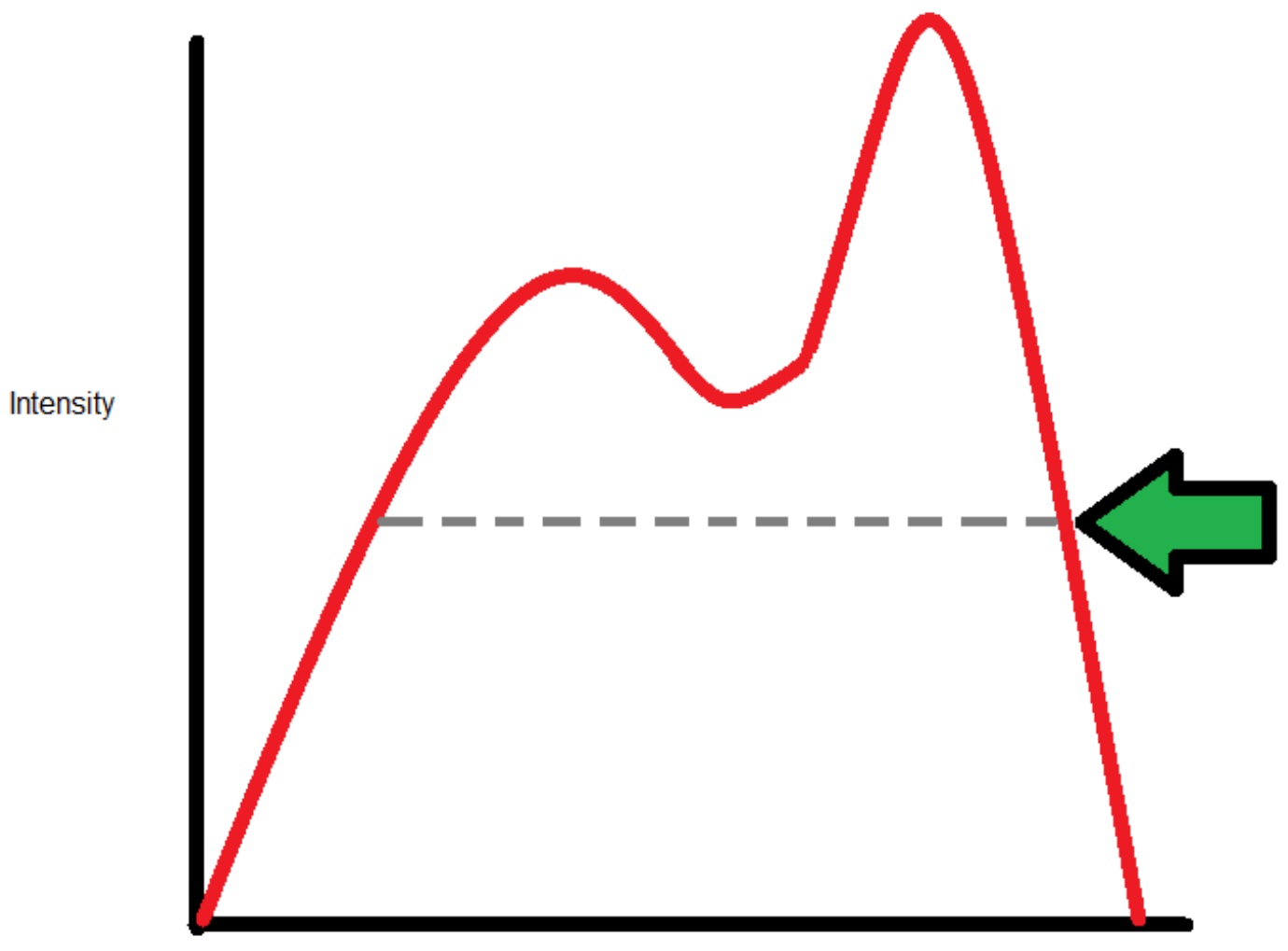

Wavelength

Figure 15: A representation of the full width at half max is indicated by the dashed line at the green arrow. The half maximum is of the higher peak. This value is less representative than if there was a single peak.

\subsubsection{Results of Change in Quantum Dot Fluorescence over 4 Days}

There was relatively little change in either the unshelled or the shelled QDs over the observational period of four days. The unshelled QDs experienced no statistically significant change in central wavelength or FWHM. The average 
fluorescence values fell within one population standard deviation. The central wavelength and FWHM remained within $1 \mathrm{~nm}$ of the first day's measurement. The average central wavelength and FWHM of shelled QDs showed no statistically significant changes.

The only statistically significant change over the four days was the intensity of the shelled QDs. The intensity of the shelled QDs increased an average of another 4088 counts. The average population standard deviation of these measurements was 90.7 counts. This translates to an additional $71 \%$ increase in intensity compared to the initial shelling, or a $531 \%$ increase compared to the unshelled sample counterparts.

\subsection{Analysis of Fluorescence Changes}

\subsubsection{Analysis of Initial Change in Unshelled to Shelled State}

All three fluorescence characteristics observed experienced changes going from the unshelled to shelled state. As literature predicted, the intensity of the fluorescence increased. This is likely due to the charge separation created with shelling. This helped validate that the shelling procedure was effective. The general small increase in central wavelength can be attributed to the subtle changes in electron excitation pathways as well as the increase in QD size. The 0 second samples likely reacted differently than the other QDs because the nucleation and growth of the QDs was in a significantly earlier stage compared to the other QD samples. The final altered fluorescence property of FWHM did 
experience an undesirable increase. An increase in FWHM is undesirable, as it indicates that the QDs are fluorescing a wider range of wavelengths. This would be detrimental when trying to use them as specific optical markers. Because the increase was small, and because the intensity increased so drastically, a shelled QD is more desirable after the initial shelling.

\subsubsection{Change Over 4 Days: Shelled}

It is likely that the intensity continued to increase on average over the course of the three days as reactants in the solution continued to slowly bond to the QDs. Along with this, the QD structure may have reached a more stable state. In the initial shelling process, bonds may have been imperfect and in partially-stable bonds. These bonds had time to move to more stable states where there was less stress on the lattice, making the QDs more uniform, improving the shell by bonding more directly with the cores. The central wavelength and FWHM remained statistically the same as the reactions were small enough as to not alter the size of the QDs to where these properties would fluctuate.

\subsubsection{Change Over 4 Days: Unshelled Cores}

Unlike the shelled QDs, the unshelled QDs may have experienced only few more unshelled QDs forming and even some decomposing. The shelling precursor already had nucleation points in the form of the QD cores, the imperfectly-shelled QDs, and the shelled QDs. To increase intensity for

unshelled QDs, more QD cores would need to form without interfering with 
existing QDs' absorption and emission of photons. Nucleation requires high energy so that the Cd and Se ions can begin to bond. This was not present when the QDs were at room temperature between tests.

\subsubsection{Extractions at 0 Seconds}

The 0 second extractions often acted differently than the other extraction times. While there were only 4 samples of 0 second extractions in total, their fluorescence characteristics were often so drastically different than the other QDs that it warranted notice. This is because more time is needed for QD core nucleation and growth. More time will help a uniform size of QD core to form as the compounds begin to mix more evenly through the suspension. The 0 second QD cores often experienced multiple similar-size peaks. This means that there were multiple sized of QDs present. There was too much variability in fluorescence compared to the other samples. The shelling procedure often amplified the undesirably unique nature of the 0 second extraction fluorescence. The 0 second extraction proved to be too unpredictable and unreliable to produce consistent fluorescence trends.

\subsection{Conclusion}

This experiment provided a multitude of useful information. The 0 second extraction will be omitted from future synthesis due to its unreliable and unpredictable nature. While there was a slight undesirable increase in shelled QD FWHM, it is relatively small and would not drastically affect biomarker quality. 
The shelled QDs will be used in further experimentation because of the significantly higher intensity than the unshelled QD cores. The increased intensity would be easier to detect either by visual observation or by a scanning device.

No particular time interval was observed to output a specific wavelength by any statistically valid scale. The experiment was not powerful enough to detect differences in extraction times as there were only two samples per extraction time of unshelled and shelled. Any observed differences could possibly be within the scale of human error.

\subsection{Additional Recommendations}

If this experiment were to be repeated, it could be improved. An increase in replicates past two batches would simultaneously improve accuracy of results as well as allow for testing of individual time intervals. It would also be useful to test a wider range of extraction times, as it appeared that QD cores did change in fluorescing central wavelength as the extraction times reached greater differences. The potentially larger QDs could react differently to the shelling process. A wider range of time may reveal further changes to both the unshelled and shelled QDs. The times selected would be varied based on how long the QDs are to be kept before processing into QD constructs. This could be on the scale of multiple weeks or months. 


\section{Chapter 3 - Synthesis of Quantum Dot Constructs}

\subsection{Introduction}

As discussed in section 1.4.4, QDs hold great promise as a biomarker. Compared to most organic dyes and fluorescent proteins, QDs are brighter and more resistant to photobleaching (8). Their tunable emission wavelength allows for customization for multiple purposes. Multiple sizes/colors of QDs could even be used simultaneously to identify different points of interest. They are not without fault though. As mentioned in 1.4.5 QDs have significant problems to overcome. The most immediate problem QDs have as biomarkers is their hydrophobic nature. The colloidal syntheses method employed leaves the QDs surrounded in a passivating layer TOP. While this layer is meant to prevent QD conjugation and encourage monodispersity in the colloid, it can be utilized to reverse the hydrophobic nature of the QD. By interdigitating the chains of a phospholipid with the chains of the TOP coating, a QD can be turned from a hydrophobic particle into a functional hydrophilic QD biomarker micelle (34). Because the QD is now surrounded by the polar heads of the phospholipids, and connected via the interdigitated chains of the phospholipid tails and TOP chains, the QD can be immersed in water without rejection. The methods of encapsulation were based upon previous similar processes utilized by Teresa Pellegrino, Liberato Manna, et.al. from Ludwig Maximilians Universität München, University of Bari, and the National Nanotechnology Lab of INFM (49). It is hoped that the following encapsulation method will be further researched at Cal Poly and potentially applied for use as a biotag in research. 


\subsection{Preparation for Encapsulation}

The synthesis and shelling of the CdSe/ZnS QDs left the QDs suspended in octadecane $(\mathrm{ODE})$. ODE, chemical formula $\mathrm{C}_{18} \mathrm{H}_{38}$, is a straight chain molecule from the alkane family (50). Alkanes are insoluble in polar solvents such as water (51). This is useful in keeping the nonpolar QDs well distributed throughout the suspension, but is not useful in making the phospholipids soluble. Phospholipids are highly soluble in organic solvents such as chloroform, acetone, and benzene (52). In order to facilitate QD encapsulation with phospholipids, the QDs needed to be suspended in such a substance. This was achieved by precipitating the QDs out of ODE and resuspending them in chloroform. The precipitation method utilized centrifugation of the ODE suspended QDs with an absolute 200 proof (greater than $99.9 \%$ purity) ethanol (EtOH) mix (53). The QDs are completely insoluble in $\mathrm{EtOH}$. Due to this aspect of $\mathrm{EtOH}$, and the fact that $\mathrm{EtOH}$ has a higher density of $0.790 \mathrm{~g} / \mathrm{mL}$ compared to ODE's density of $0.777 \mathrm{~g} / \mathrm{mL}, \mathrm{EtOH}$ acts as a useful means of separating out the QDs to create a "pellet" of QDs at the bottom of the centrifuge tube (54). The QDs are the heaviest substance, and are pulled to the bottom of the tube. Because the $\mathrm{EtOH}$ is denser than the ODE, it is pulled below the ODE, forming 2 layers. The QDs are then forced to mix with the $\mathrm{EtOH}$, as they are the two densest substances. This separates the QDs from the ODE. 


\subsubsection{Quantum Dot Precipitation Method}

Though the centrifuge could hold one to six tubes at a time, in most instances, two tubes of equal weight were used. A portion of ODE suspended QDs were added into each tube (e.g. 3mL of QD-ODE suspension and $6 \mathrm{~mL}$ of $\mathrm{EtOH})$. EtOH was then added in two parts $\mathrm{EtOH}$ to one part QD-ODE suspension. In the instances of uneven samples of QD-ODE suspension (where one sample vial held more volume of QD-ODE suspension than another vial), $\mathrm{EtOH}$ was added in the same 2:1 ratio for the larger sample, and $\mathrm{EtOH}$ was added to the smaller sample until the mixtures were of the same volume. For example if one sample held $2 \mathrm{~mL}$ of QD-ODE suspension, and another vial held 3mL of QD-ODE suspension, and both were to be centrifuged simultaneously, different amounts would be added to each. The $3 \mathrm{~mL}$ vial would have the standard 2:1 ratio of EtOH: QD-ODE at $6 \mathrm{~mL}$ of $\mathrm{EtOH}$ added. The $2 \mathrm{~mL}$ vial would have $7 \mathrm{~mL}$ of $\mathrm{EtOH}$ added so that both vials would hold $9 \mathrm{~mL}$ of QD-ODE-EtOH mixture. This was done to promote balance within the centrifuging process.

With the initial addition of $\mathrm{EtOH}$, the QDs remained separated, forming spheres of ODE-QD mix in a similar appearance to how oil separates in water. To encourage a more even solution, $\mathrm{EtOH}$ was injected more forcefully into the liquid. Once at the proper total volume, the vials were capped and then shaken in order to agitate the solution to encourage mixing more evenly. Without this agitation step, the surface tension of the QD-ODE solution spheres would often remain separated, leaving the QDs suspended in ODE spheres at the base of the vials. 
The appropriately portioned and agitated vials were then loaded into opposite sides of the centrifuge so as to balance the machine. The vials were spun within the centrifuge at $4000 \mathrm{rpm}$ for 10 minutes, in accordance with previous project methods. This was considered one "cycle" of centrifuging. After one cycle, the clear supernant was removed, leaving a liquid mixture of $1 / 2$ to 2 ML of QD-ODE solution. More EtOH was added in the same volume as the initial addition. This mixture was once again agitated, and the vials were balanced. A second cycle of $4000 \mathrm{rpm}$ for 10 minutes was run. After the second cycle, if a QD pellet had formed at the base of the vial, the EtOH and ODE were extracted and then poured off of the QDs. On some occasions, a $3^{\text {rd }}$ centrifuging cycle was required in order to separate out the QDs well enough to remain in the vial without being poured away with the $\mathrm{EtOH}$. The samples were considered ready for EtOH removal when the QDs had formed into a highly viscous, or solid mass at the tip of the vial that would not flow out of the vial as the liquid was poured away.

Once the QDs were isolated in the vial, they were placed in an active fume hood for at least twenty-four hours so that any lingering EtOH would evaporate out of the vials. This was the final stage of the QD isolation process. After the drying period, the only remaining substance in the vials were the isolated QDs. Though previous methods of QD precipitation had required occasional removal of abnormal black precipitates, none were encountered in the trials run for this thesis. 


\subsubsection{Quantum Dot Suspension into $\mathrm{CHCl}_{3}$}

Once the QDs were isolated, they needed to be resuspended into a solvent which could both hold the non-polar QDs and dissolve the solid phospholipid powder. Chloroform $\left(\mathrm{CHCl}_{3}\right)$ served to satisfy this requirement. Unlike the $\mathrm{ODE}, \mathrm{CHCl}_{3}$ was able to dissolve the phospholipids as it is significantly more polar then the ODE. It still maintained enough non-polarity to act as a temporary solvent for the QDs without $\mathrm{QD}$ coalescence. $\mathrm{CHCl}_{3}$ was added in a minimum volume of equal the original QD-ODE solution (amount of QD-ODE added to the centrifuge vial). A greater amount of $\mathrm{CHCl}_{3}$ was often beneficial for generating more samples for further stages in experiments.

\subsection{Calculations Required for Phospholipid Encapsulation Process}

To encapsulate the QDs in phospholipids, it was desirable to have a known ratio of phospholipids to the total QD surface area in the QD- $\mathrm{CHCl}_{3}$ solution. In order to calculate this ratio, several variables needed to be analyzed and calculated prior to phospholipid addition. The total QD surface area in the solution was determined by utilizing the known aspects of the QD solution. These known factors were the excitation wavelength, the weight of the QDs, and the concentration of the QDs in the $\mathrm{CHCl}_{3}$.

\subsubsection{Quantum Dot Size}

One of the first variables to calculate to determine the total surface area on the QDs in the solution was the size of an individual QD. W. William Yu, et al. 
at the University of Arkansas experimentally determined an equation to estimate the size of a CdSe QD from the fluorescing wavelength (55). This equation is displayed in Equation 1, where $D$ is the average particle diameter in nanometers, and $\lambda$ is the fluorescing wavelength of the QDs.

$$
\begin{gathered}
\text { Equation 1: Diameter }=\left(1.61222 \mathrm{E}^{-9}\right) \lambda^{4}-\left(2.6575 \mathrm{E}^{-6}\right) \lambda^{3}+\left(1.6242 \mathrm{E}^{-3}\right) \lambda^{2}- \\
\left(4.277 \mathrm{E}^{-1}\right) \lambda+(41.57)
\end{gathered}
$$

For example, a QD solution that emits a wavelength of roughly $549 \mathrm{~nm}$ should have QDs with an average diameter of $3.02 \mathrm{~nm}$. The diameter can be used to easily determine the radius of the approximately spherical QD. By modeling the QD as a sphere, a rough estimate of the average surface area of a QD can be calculated by utilizing the equation for the surface area of a sphere as seen in Equation 2.

\section{Equation 2: Surface Area $=4 \pi r^{2}$}

This gives the approximate surface area on an average-sized QD in a solution. This is an important step in determining the total surface area available on QDs in a QD solution. Another critical piece of information missing is the total number of QDs in the solution. By knowing the surface area on one $Q D$, and knowing the approximate total number of QDs in the solution, the total surface area available on all QDs can be determined by simply multiplying the two values. 


\subsubsection{Quantum Dot Quantity}

Calculation of QD quantity starts far before the QDs are ready to be mixed with phospholipids. In order to determine the quantity of the QDs, the average volume of QDs must be known, as well as the total weight of the QDs. To determine the average volume of a QD, Equation 1 can prove useful again. By measuring the fluorescing wavelength of the QDs, the average diameter can be calculated. The diameter indicated the radius as well. By once again modeling the $\mathrm{QD}$ as a sphere, a rough estimate of the average volume of a $\mathrm{QD}$ can be calculated by utilizing the equation for the volume of a sphere as seen in Equation 3.

$$
\text { Equation 3: Volume }=\frac{4}{3} \pi r^{3}
$$

This gives the approximate volume of an average-sized QD in the solution. This is an important step in determining the total quantity of QDs in a QD solution. The volume of a single QD is not useful alone. The weight of a single QD is important.

By assuming the QD sphere model is entirely CdSe, the mass of a single QD can be calculated. Because density is mass divided over a set volume, and the average volume of the QD is known, by multiplying the density of CdSe by the volume, the mass of a single QD can be determined. All that is needed after this is to find the total weight of all of the QDs in the solution. Once the total weight of all of the QDs is known, the approximate total number of QDs can be 
determined by dividing the total weight of all QDs by the mass of a single QD. The last piece missing before this calculation can be performed is the total weight of all of the QDs. This can't be determined by the fluorescence, or by simplifying the model. It must be measured.

Measurement of total QD weight is most easily performed before and after the centrifuging process. To find the total weight of QDs in the centrifuging vial, it is simpler to measure the change in the centrifuge vial's weight with the QDs within, rather than to try and remove, weigh, and replace the QD pellet. To do this, the empty vials must be weighed before any QD solution is added. It was also important to keep the caps of vials matched with the vials so that any minor differences in cap weight did not skew the weight of the vials before and after QDs were isolated inside of them.

The process for weighing out the QDs was simple. The empty centrifuge vial would be weighed and the value recorded. The QD-ODE solution would be added, and centrifuged in the process described in section 3.2.1. Once the vials were dry, with the QD pellet inside, they were weighed once again. The initial weight of the empty vial would be subtracted from this new weight of the vial with the QDs within. The resulting difference would be the approximate mass of all of the QDs in the vial. This was considered to be the total weight of all QDs. While the concentration of QDs would change, the total weight of the QDs would not.

Once the weight of all QDs was determined, along with the approximate average weight of a single QD, the total quantity of QDs in the vial was simple. By dividing the total weight of the QDs by the weight of a single QD, the number 
of QDs could be calculated. Due to the incredibly small size of the QDs, it was not abnormal to have estimates in the hundreds of quadrillions, or X.XXXe17. Significant figures were observed, making the estimates roughly accurate down to the $10^{14}$ scale (e.g. $\left.665,700,000,000,000,000\right)$.

\subsubsection{Quantum Dot Concentration and Total Surface Area}

Once an approximation of the total number of QDs had been determined, then the QD concentration can be determined. Previous procedures added the same quantity of $\mathrm{CHCl}_{3}$ as the ODE (56). By regarding the ODE solution as the set minimum, the QDs would have a highly similar concentration to when they first formed. This may be regarded as desirable as it is how the QDs spontaneously formed without being overly concentrated in the solution. While this was regarded as the minimum amounts, it often roved beneficial to have a larger quantity of solution, even at the cost of a reduced concentration. By increasing the total number of samples which could be generated by each vial of QDs, the variable of "QD size" could be essentially eliminated. Vials were filled to the $10 \mathrm{~mL}$ mark near the top of the vial. This was roughly $3-5 \mathrm{~mL}$ more $\mathrm{CHCl}_{3}$ than the previous fluid of ODE. The diluting of the QDs from the previous ODEQD concentration would not be a significant problem as the specific concentration of QDs in the solution needed to be calculated regardless. No value of $\mathrm{QD}$ concentration in the ODE-QD solution had been calculated before this step, so resuspending the QDs in the same volume of $\mathrm{CHCl}_{3}$ as ODE would not be important. 
To calculate the concentration of QDs, first a set amount of $\mathrm{CHCl}_{3}$ needed to be added. Once the QDs were suspended in a set volume of $\mathrm{CHCl}_{3}$, the concentration could be calculated by dividing the total number of QDs by the total volume of the $\mathrm{CHCl}_{3}-\mathrm{QD}$ solution. This solution could then be divided into several samples for further testing of phospholipid encapsulating.

\subsubsection{Phospholipids per Square Nanometer of Quantum Dot Surface Calculation}

There were two main variable factors in determining the amount of phospholipids to add to each sample. These variables were the type of phospholipid added and the desired number of phospholipids to cover each QD surface. Each hold a major factor in determining the weight of phospholipid powder to add into the solution.

As the phospholipids were in a powder form, and not in solution, the best way to quantify how many phospholipids were being added was to utilize the molecular weight of an individual phospholipid molecule. The molecular weight of the phospholipid indicates the weight of a single phospholipid molecule. This can be utilized to calculate approximately how many molecules are in a given mass of phospholipid powder. One experimental variable was the type of phospholipid being used. As the differing phospholipids had distinct molecular weights, a different weight of phospholipid powder would need to be added to a sample to achieve the same concentration of phospholipids per square nanometer of QD surface. 
Another experimental factor which was tested was the desired number of phospholipids to add per square nanometer of QD surface. To vary this factor, the concentration of phospholipids added to the concentration of QDs needed to be considered. A higher concentration of phospholipids compared to the QDs in the solution would theoretically provide more phospholipids to attach to each individual QD. A lower concentration would similarly have fewer phospholipids to attach to each QD. By varying the concentration of phospholipids added to the solution, the amount of phospholipids per surface was altered. Because the size of a sample was controlled, and the concentration and size of the QDs within the sample were already set, the simplest way to alter the concentration of phospholipids compared to the QDs was to alter the amount of phospholipids being added to a sample. To do this, different quantities of phospholipids would need to be added. This was quantified by adding different weights of phospholipid powder. Once again, the quantity of phospholipid molecules added could be determined by using the molecular weight for each type of phospholipid.

The final equation for determining how much phospholipid powder to add took the form of Equation 4, where $N_{A}$ is Avogadro's number. The experimental factors altered in testing have been bolded.

\section{Equation 4:}

Weight of Phospholipids to Add= Total Surface Area of QDs in Sample $\times$

\section{Desired Number of Lipids per $\mathbf{n m}^{2}$ QD Surface Area-}

\section{$N_{A} \times$ Molecular Weight of Phospholipid}


The "Total Surface Area of QDs in Sample" was measured in "nm", the "Desired Number of Lipids per square nanometer of QD Surface Area" was measured in " $1 / \mathrm{nm}^{2}$ ", by dividing by $\mathrm{N}_{\mathrm{A}}$ the units were the same as multiplying by "mol", and finally the molecular weight was measured in " $\mathrm{g} / \mathrm{mol}$ ". These units reduce to result in a mass in grams to add to the sample.

\subsection{Phospholipid Encapsulation Process}

Once the calculations have yielded the amount of a specific phospholipid to add to a sample of $\mathrm{CHCl}_{3}-\mathrm{QD}$ solution, the encapsulation process can be performed. There are three major steps in the encapsulation process: the weighing and addition of the phospholipids to the sample, a rotovaping procedure, and resuspension in water with buffer.

\subsubsection{Weighing and Addition of Phospholipids}

The phospholipid powder was stored in a freezer so as to preserve the phospholipids from breaking down. Once the appropriate weight of powder had been calculated for a sample, the phospholipids were measured out on a scale as accurately as possible. The powder was then transferred into an empty round bottom flask. The transfer was best accomplished by utilizing an inert paper. The paper was folded in half diagonally before being placed on the scale. The scale was then set to zero with the paper on it to compensate for the new weight. The phospholipids were then spooned onto the paper utilizing a stainless steel chemistry spatula. Once at the appropriate amount, the whole paper containing 
the phospholipids could be lifted from the scale. The paper could then be twisted into a funnel shape so that as much of the phospholipids as possible could be knocked into the flask without spilling. After the phospholipids were in the flask, the predetermined amount of $\mathrm{CHCl}_{3}-\mathrm{QD}$ mix (as used to calculate the quantity of phospholipids needed) was added into the flask. The flask was then closed and agitated to encourage an initial mixing of the solution to become more evenly dispersed. Just shaking the flask was not adequate though.

\subsubsection{Rotary Evaporation}

After the $\mathrm{CHCl}_{3}-\mathrm{QD}$-phospholipid sample had been prepared, it was moved to a rotary evaporation machine similar to the one pictured in Figure 16. The round bottom flask acted as the "pot" in the figure. The flask was locked into place with a clip before a vacuum was placed onto the system, sealing it shut. A motor was then turned on to rotate the flask. This rotation kept the mixture constantly agitated so that no moisture would be trapped beneath a layer. The final evaporated substance would form a thin film on the internal walls of the flask, rather than accumulating only at the base. The vacuum encouraged rapid evaporation of the $\mathrm{CHCl}_{3}$ as well. This even and rapid evaporation was desirable so that the QD-phospholipid constructs (QDCs) could form throughout the mixture, in a timely manner, with as little $\mathrm{CHCl}_{3}$ left in the substance as possible. The formation of QDCs was further promoted during the resuspension into a polar substance. 


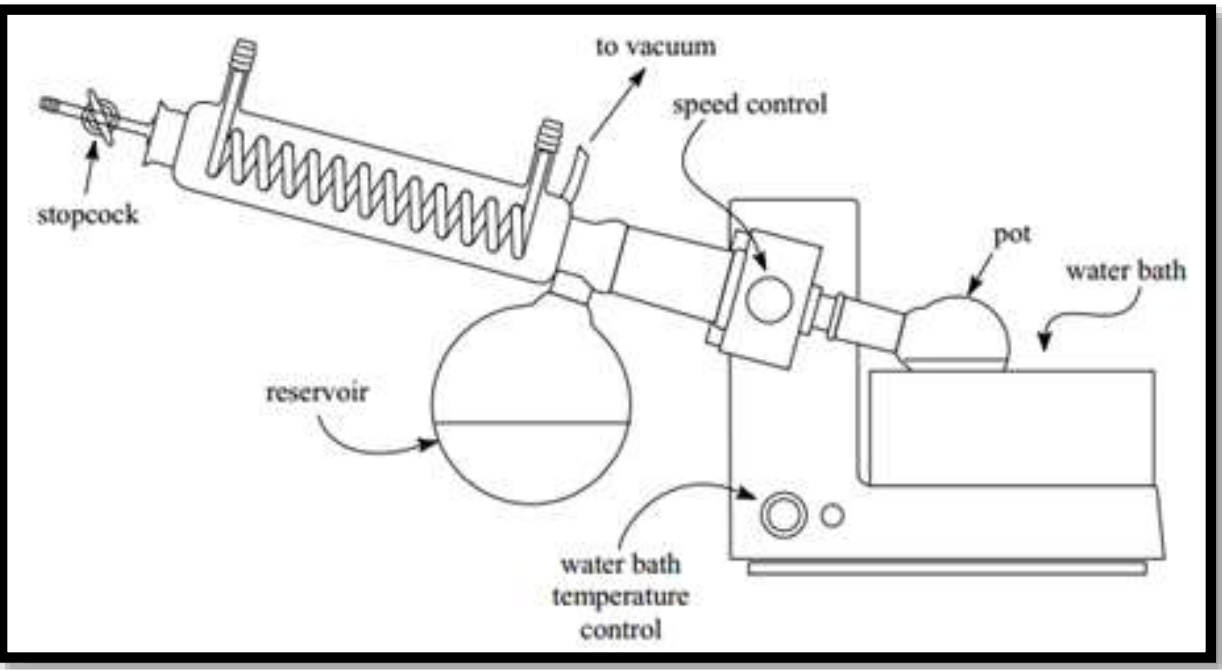

Figure 16: A schematic of a typical rotary evaporation machine. The QDs in the "pot" rotate in a water bath while a vacuum is induced on the interior to accelerate evaporation. (57)

\subsubsection{Resuspension in Water and Buffer}

After evaporation, the QDCs (and remaining bare QDs and unattached phospholipids) were ready for resuspension into a protic solvent. The solvent chosen was a mixture of water with a tris-borate buffer $(0.5 \times$ Tris-Borate-EDTA (TBE, Sigma-Aldrich \#T-3912)). This buffer would act to further solubilize the remaining phospholipids so that they would better attach to the remaining bare QDs. For consistency, $3 \mathrm{~mL}$ of this buffer solution was added to every evaporated QDC sample. This final suspension was then capped and agitated via shaking before being immersed into a sonicator for 30 minutes. The sonicator acted to further agitate the samples, dispersing the nanoparticles in the liquid, promoting more exposed QD surfaces for which the loose phospholipids could adhere. After this final stage, the QDC suspension was taken in for spectral characterization. 


\subsection{Experimental Procedure Variations for Quantum Dot Encapsulation}

Though Section 3.4 states the procedure of how to perform the process, it does not indicate the specific factors altered and tested through the experiments performed, nor the testing methods utilized. Both full samples, and partial samples were tested in an effort to observe different characteristics of the solutions. Two types of phospholipids were tested, one factor at a time altered, it does not indicate the specific alterations that occurred throughout the experimentation. The main variables were the type of phospholipid used and the desired phospholipid concentration per square nanometer.

\subsubsection{The Two Sample Extraction Types}

There were multiple variations of extractions performed on the samples for testing. A "full-sample" method and a "partial-sample" method were both utilized. A full-sample extraction transferred the entire contents of a given flask of QDs which had gone through the encapsulation process, and loaded these contents into a cuvette for testing. The full-sample testing accounted for observing all components of the given solution. A partial-sample selectively removed a set amount $(2 \mathrm{~mL})$ of the solution from the top of the solution downwards, removing the uppermost portion of the sample within the flask. This was done in an attempt to extract only the suspended particles in the top layer of the solution. Each method yielded unique results. 


\subsubsection{The Two Phospholipid Types}

The two phospholipid types used were both variants of phosphocholine. One type was DPPC, and the other was DSPC. Though they had almost identical chemical composition, but the chains composing the phospholipid "tails" were of different lengths. This caused them to be different molecular weights. Because DSPC has longer carbon chain tails, it had a higher molecular weight $(790 \mathrm{~g} / \mathrm{mol})$ than DPPC $(734 \mathrm{~g} / \mathrm{mol})(38)$. A model of these chains can be seen in Figure 17, with DPPC (molecular formula $\mathrm{C}_{40} \mathrm{H}_{80} \mathrm{NO}_{8} \mathrm{P}$ ) on the left, and DSPC (molecular formula $\mathrm{C}_{44} \mathrm{H}_{88} \mathrm{NO}_{8} \mathrm{P}$ ) on the right.

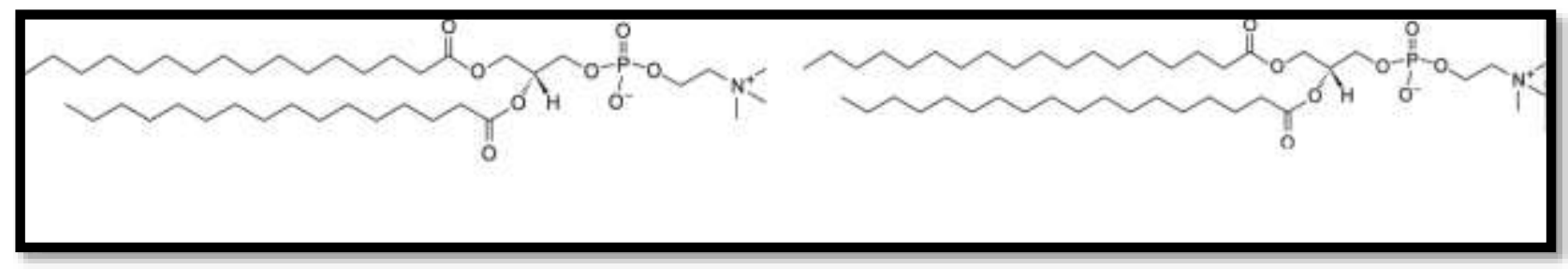

Figure 17: Two variations of the phosphocholine phospholipid. (Left) DPPC has a shorter tail chain length than DSPC (right). (38)

The first variable selected for testing was the type of phospholipid to be used. It was selected as the first variable due to available quantities of phospholipids, and reducing the total number of tests. By testing the two types first, less total samples needed to be prepared, which saved time, QDs, phospholipids, and by extension money. There was also the added benefit of being able to use one specific size of QD for testing both types of phospholipids. Limiting the samples to one average size of QD eliminated variability between tests. 
The first experiments compared a control group of QDs without phospholipids, QDs with DSPC at a concentration of three phospholipids per square nanometer, and QDs with DPPC at a concentration of three phospholipids per square nanometer. By keeping the concentration set at three phospholipids per square nanometer, any differences between measured values would be isolated to the difference in the phospholipids. Trials were run for each, and it was decided that the smaller DPPC was the superior phospholipid for the process. The reasoning for this and results of the experiments will be discussed in Chapter 4.

\subsubsection{Altering the Concentrations of DPPC}

The first trial of DPPC tested the phospholipids at a concentration of three phospholipids per square nanometer. This trial remained useful to compare to the experiments of the DPPC-specific tests. The second set of experiments focused on varying the concentrations of DPPC. Two new concentrations were selected to test both greater and lesser concentrations of phospholipids. If fluorescent properties proved better by either increasing or decreasing the concentration, then further experiments could be directed towards increasing or decreasing the quantity of phospholipids. The two new concentrations selected were ten phospholipids per square nanometer, and one phospholipid per square nanometer. Along with these two concentrations, a second control of purely DPPC was generated, using the equivalent amount of phospholipids as would be used for a concentration of ten phospholipids per square nanometer of QD surface. 


\subsection{Fluorescence Characterization}

The QDC suspension fluorescence was measured utilizing the same process documented in section 2.7. The fluorescence values observed were compared to the corresponding values of the QDCs in their QD-ODE stage. The QD- $\mathrm{CHCl}_{3}$ stage had been shown to have nearly identical fluorescence to the QD-ODE stage. The comparison of fluorescence was used to identify major differences in fluorescence peaks' locations, differences in fluorescence intensity, and any new fluorescence patterns to the QDC suspension.

Samples were scanned utilizing a ten scans-to-average output in order to reduce instantaneous variability. This means that the sample was averaged over ten scans to create a single graphical output. These averaged scans were measured every 5 seconds over the course of 6 minutes, creating 72 readings of the spectrum. These outputs were then averaged to provide the average fluorescence over the 6 minute span.

To compare the phospholipids, two samples were generated, comparing DSPC and DPPC with QDs, both having a ratio of three phospholipids per square nanometer of QD surface. Once the superior phospholipid (DPPC) was selected (why it is superior in section 3.7.1), the concentration of phospholipids per square nanometer was varied using only the superior phospholipid. The new concentrations to be tested against the three phospholipid per square nanometer were one phospholipid per square nanometer, and ten phospholipids per square nanometer. These samples were also compared a control group of pure QDs, and a control group of pure phospholipids (having the same quantity of 
phospholipids as the ten phospholipids per square nanometer of QD surface sample). Each comparison yielded unique insights. An ideal QDC would have a distinct emission from the QDs, and a high signal to noise ratio at that emission. Reflected light would be minimized, and the QD fluorescence would be maximized.

\subsection{Results}

There were two areas of interest in the observed spectrum of light. The first area about the excitation wavelength $(390 \mathrm{~nm})$ indicated how much of the excitation wavelength was being observed within the sample. This wavelength was created by the excitation source of a UV laser. A lower intensity is desirable in this area. A lower intensity indicates a larger absorbance of UV light by the quantum dots. Less UV light would also be useful in application with biomarkers, as there would be less interference when trying to locate the QDs of another wavelength. The second area of interest was the wavelengths surrounding the QD fluorescence. A greater intensity in this area indicates a greater amount of QDs fluorescing. When in solution, a higher intensity of QD fluorescence can indicate a larger amount of QDs within a solution. An ideal sample would have a low UV intensity, but a high QD fluorescence.

\subsubsection{DSPC vs DPPC Fluorescence}

The first set of experiments compared DPPC and DSPC phospholipids to a control group of pure QDs that had gone through the same coating process 
without any phospholipids present in the procedure. Both the DPPC and DSPC phospholipids were utilized in concentrations of three phospholipids per square nanometer of QD surface.

The first sample type measured was the full sample. The UV spectrum seen in Figure 18. In the UV wavelength, the QD control appeared to have a higher intensity than either of the QD samples processed with DSPC or DPPC.

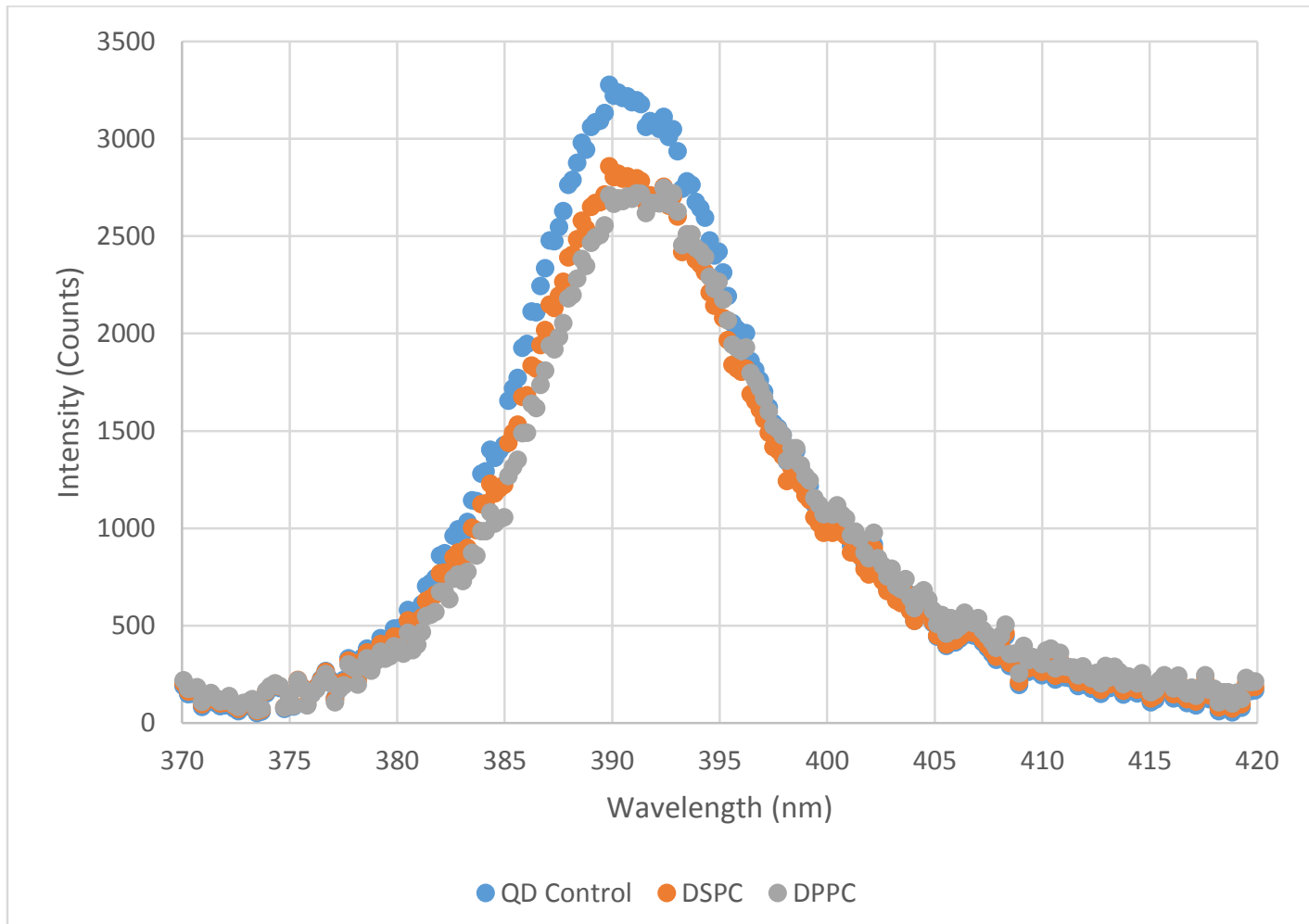

Figure 18: Full sample measurement in the UV excitation spectrum of QDs processed with DSPC and DPPC phosphocholine phospholipids compared to a QD control group processed with no phospholipids.

The QD fluorescence spectrum of the two phospholipid type tests is seen in Figure 19. In this full sampling method, there is an undesirable signal to noise ratio. It is difficult to distinguish any meaningful information from the graph. 
While it appears as though the DPPC phospholipid sample may have had the highest intensity, it is not highly distinguishable from the other samples.

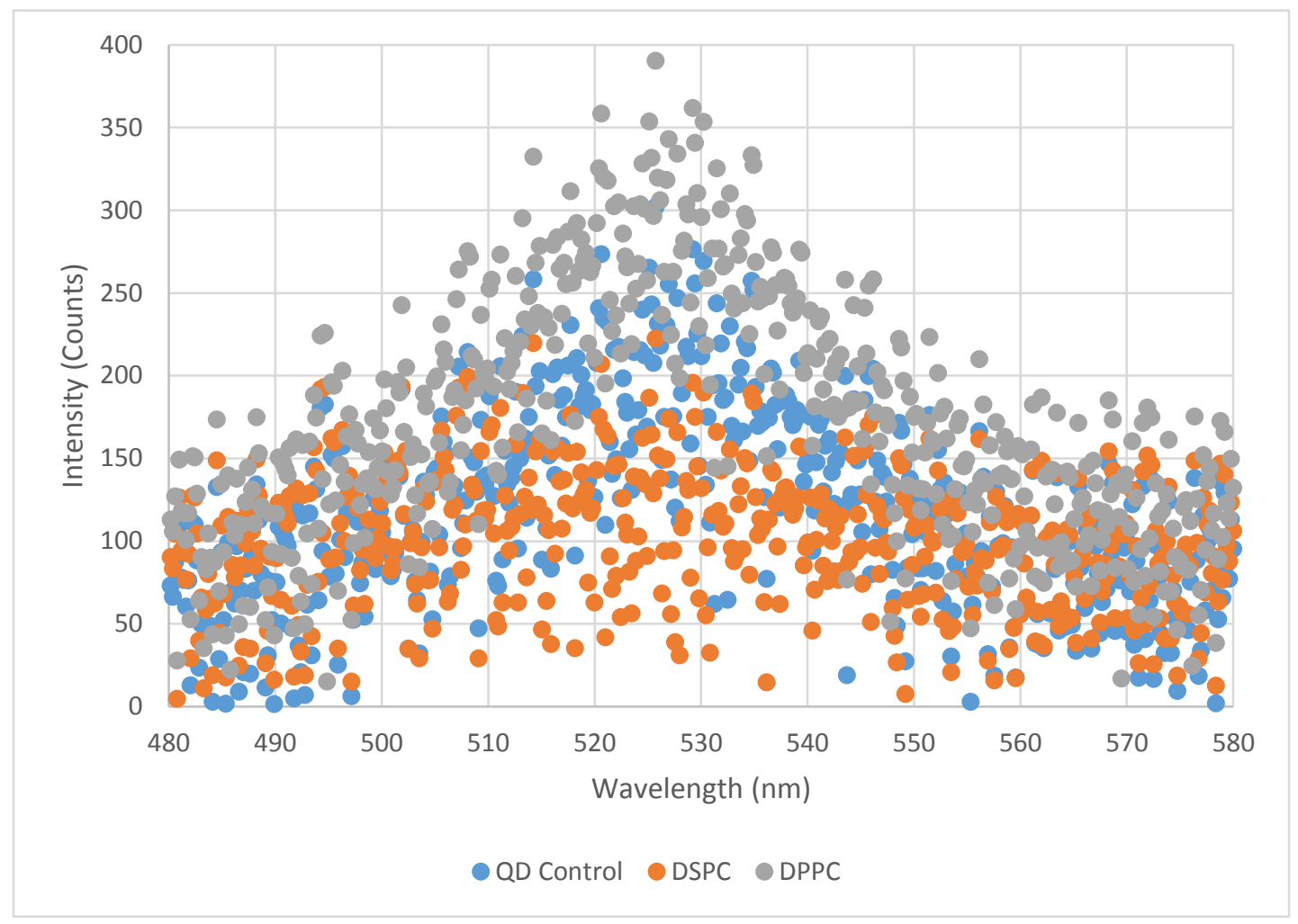

Figure 19: Full sample measurement in the QD Fluorescent spectrum of QDs processed with DSPC and DPPC phosphocholine phospholipids compared to a $Q D$ control group processed with no phospholipids. A poor signal to noise ratio is seen.

In an attempt to reduce the variability of the sample, a partial sampling method was then utilized. This process attempted to extract only QDs which were suspended in the uppermost layer of the sample. The result of the partial sample in the QD spectrum is seen in Figure 20. 


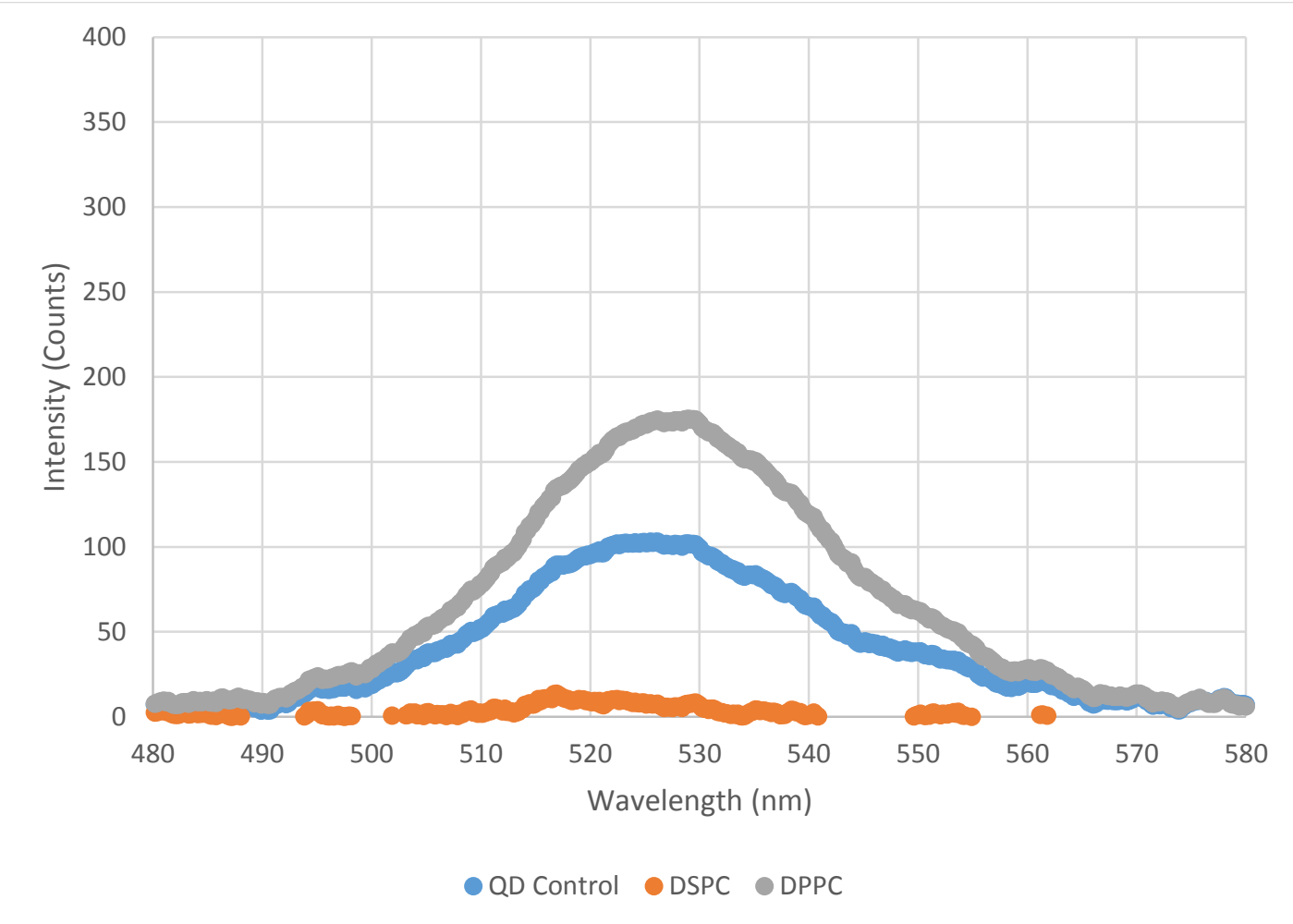

Figure 20: Partial sample measurement in the QD fluorescence spectrum of QDs processed with DSPC and DPPC phosphocholine phospholipids compared to a QD control group processed with no phospholipids. The samle processed with DSPC shows no fluorescence.

The partial sample revealed data following a clearer pattern, with a far better signal to noise ratio, with more distinguishable features for each sample. The QD control appeared to have some QD fluorescence, but the DSPC had no noticeable QD fluorescence. The sample with the highest fluorescence was the sample utilizing the DPPC phospholipids. In the ultraviolet spectrum, the partial sample yielded different results than the full sample. As seen in Figure 21, the DSPC sample had the lowest UV excitation peak, while DPPC sample had the greatest peak, even above the QD control. While a low UV peak is desirable, it was deemed more important to have a higher fluorescence. A biomarker that did not fluoresce would not be useful. The DPPC phospholipids with QDs were selected as the more desirable pairing. The DPPC phospholipids were used for 
further experimentation.

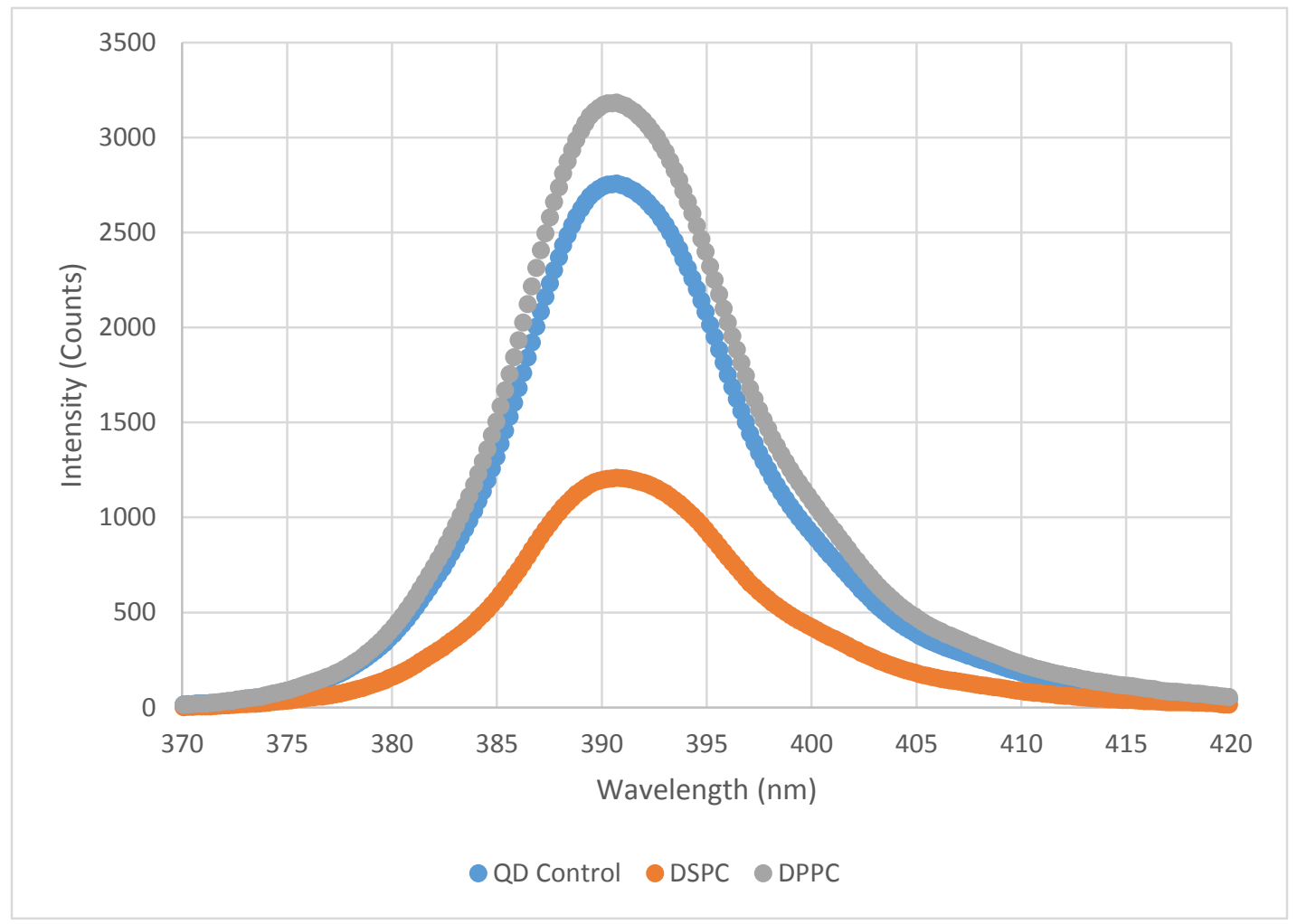

Figure 21: Partial sample measurement in the UV excitation spectrum of QDs processed with DSPC and DPPC phosphocholine phospholipids compared to a QD control group processed with no phospholipids.

\subsubsection{The Altered Concentration of DPPC}

The altered concentrations added in a new control group of phospholipids suspended without QDs. The new concentrations of one phospholipid per square nanometer, and ten phospholipids per square nanometer were then compared to the previous concentration of three phospholipids per square nanometer, and the phospholipids without QDs control. The phospholipid control was at the same concentration of phospholipids as the ten phospholipids per square nanometer sample. 
The full sample was observed first. In the ultraviolet range, there were distinct differences between the peaks of the phospholipid control, the three phospholipid per square nanometer concentration, and the one and ten phospholipid per square nanometer concentrations. As seen in Figure 22, the highest UV intensity was the phospholipid control. While the one and ten phospholipid concentrations appeared similarly low, the three phospholipid concentration was between the sets in intensity.

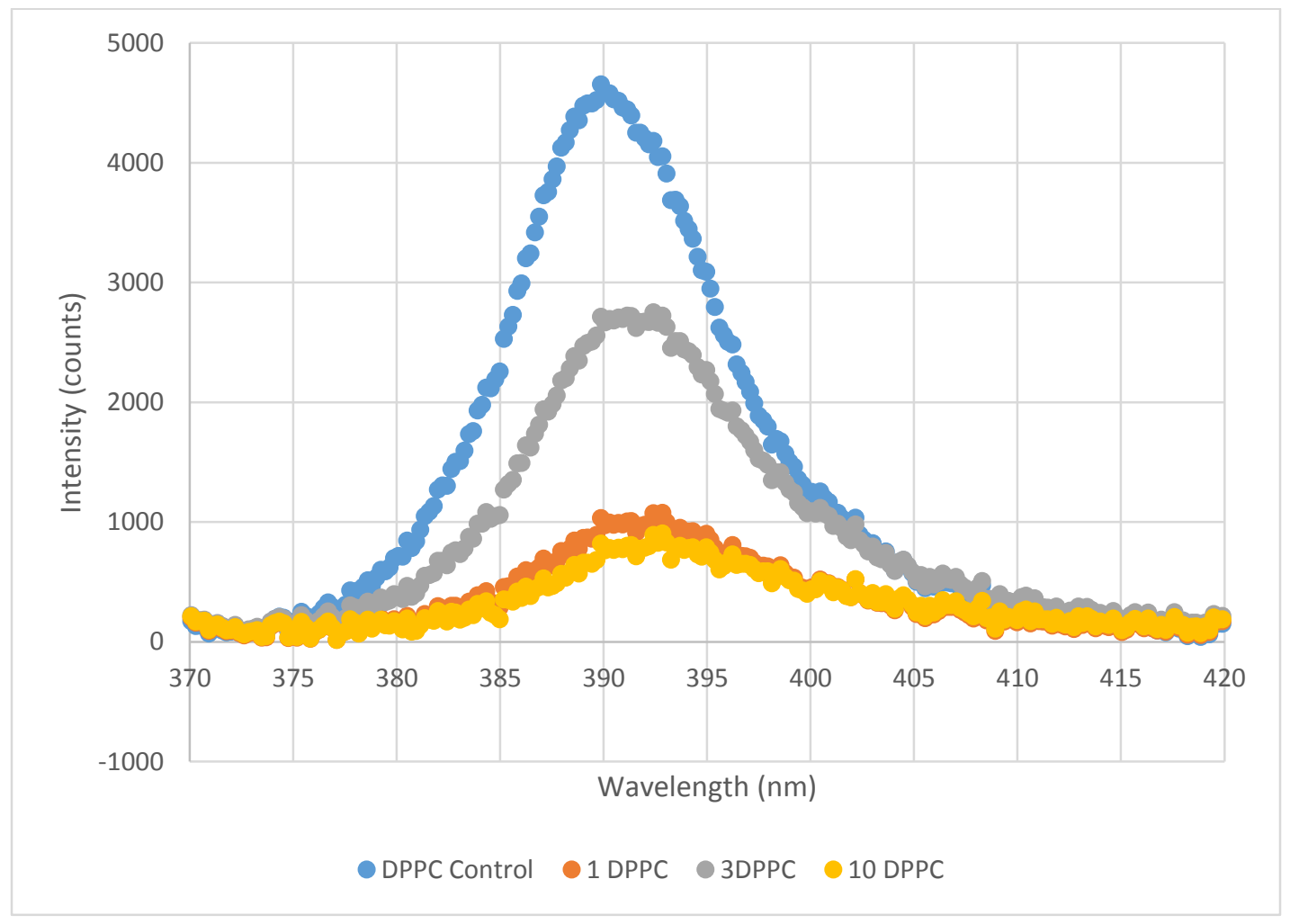

Figure 22: Full sample measurement in the UV excitation spectrum of QDs processed with various concentrations of DPPC phosphocholine phospholipids (per nm square) compared to a DPPC control group processed with no QDs.

The full sample of the QD fluorescence wavelength showed a poor signal to noise ratio once again. There was a noticeable difference between the control group of only phospholipids and the phospholipids processed with the QDs. As 
seen in Figure 23, it is difficult to distinguish a difference between the different concentrations, but the control group is different than any of the other samples.

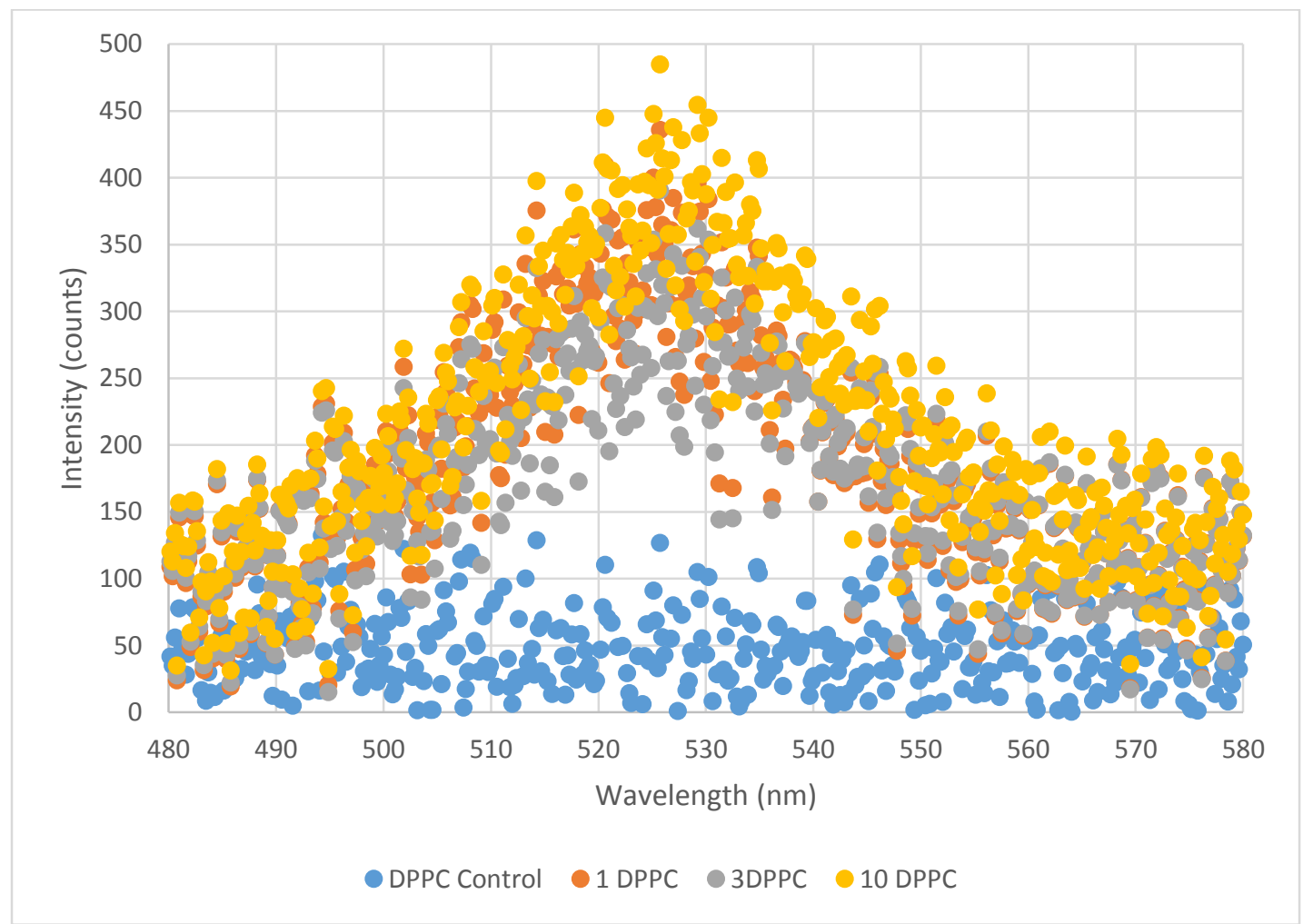

Figure 23: Full sample measurement in the QD fluorescence spectrum of QDs processed with various concentrations of $D P P C$ phosphocholine phospholipids (per $\mathrm{nm}$ square) compared to a DPPC control group processed with no QDs. The control shows little to no fluorescence

Once again, a partial sampling method was utilized in an attempt to discover potential differences between the concentrations. The partial sampling method revealed distinguishable differences between some samples. The ten phospholipid concentration had a greater intensity than either of the other phospholipid with quantum dot concentrations. All samples processed with QDs had a greater intensity than the phospholipid only control. This can be seen in 
Figure 24 .

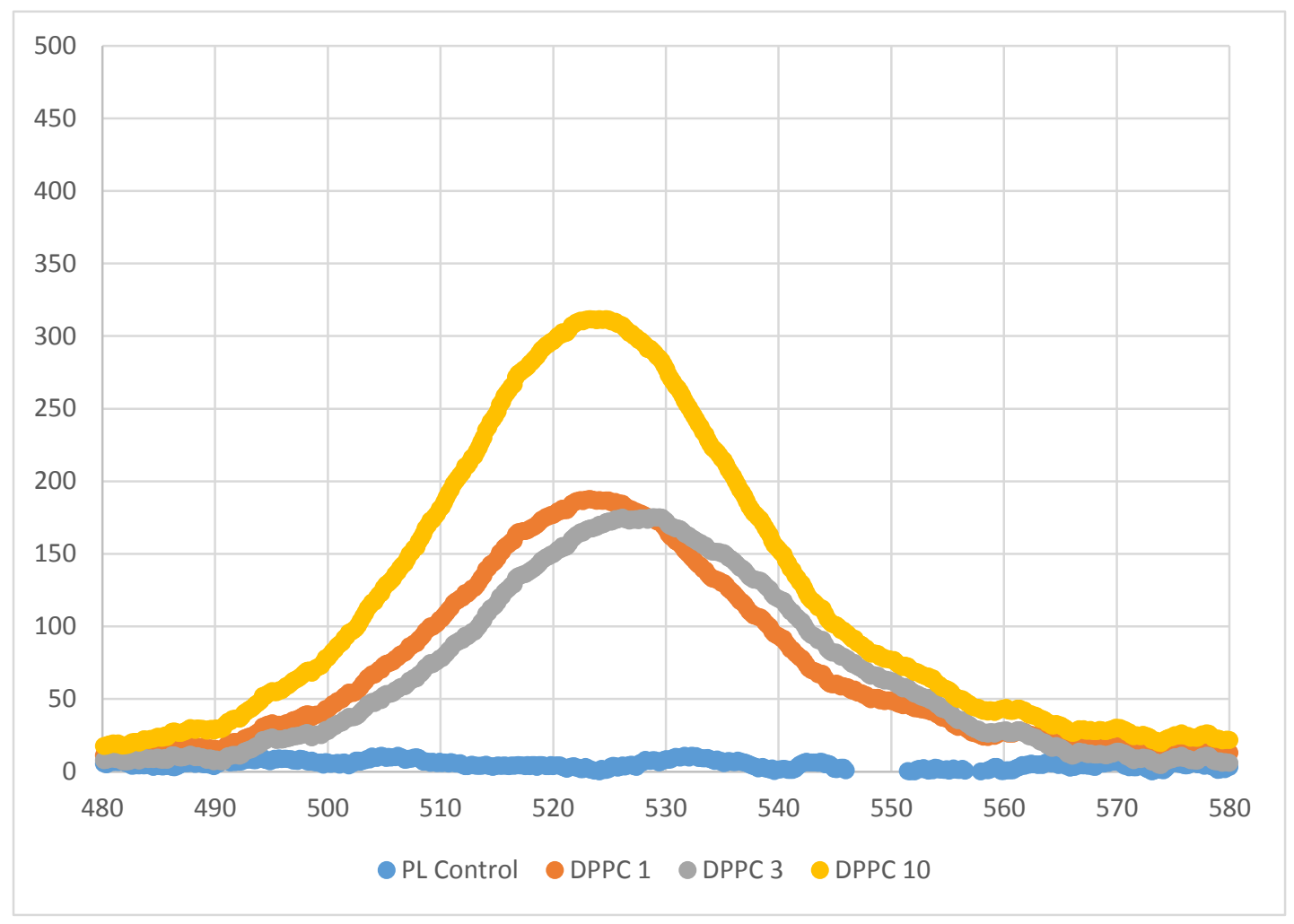

Figure 24: Partial sample measurement in the QD fluorescence spectrum of QDs processed with various concentrations of DPPC phosphocholine phospholipids (per nm square) compared to a DPPC control group processed with no QDs.

Unlinke in the tests comparing the two different phospholipids, UV spectrums of the partial and full testings were highly similar. As seen in Figure 25, the phospholipid control maintains the greatest UV intensity, with the three phospholipid concentration lower, and the one and ten phospholipid concentrations at the lowest UV intensity. 


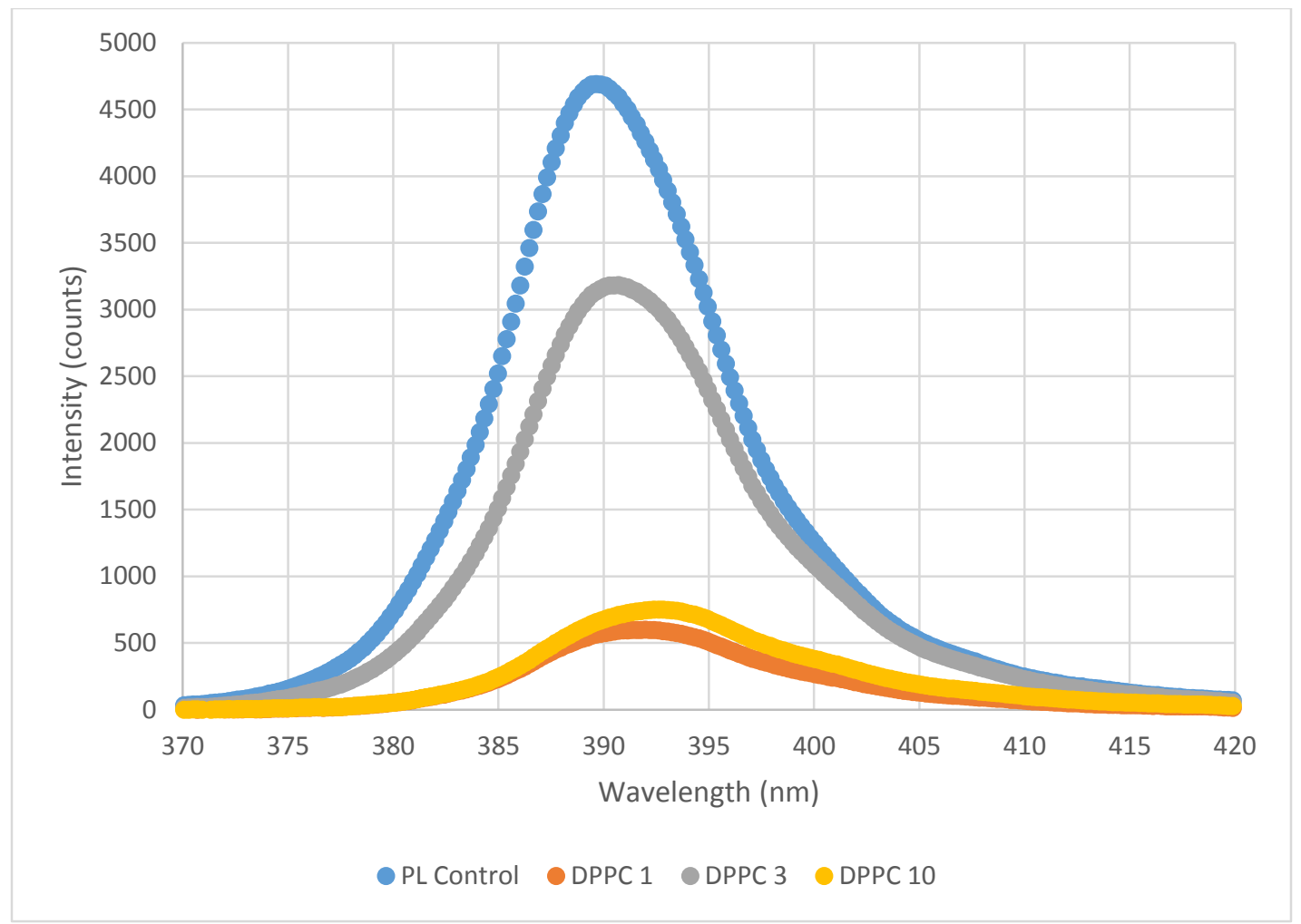

Figure 25: The partial sample values of the UV fluorescence spectrum for altering concentrations of DPPC with QDS compared to a pure DPPC control. 


\section{Chapter 4 - Discussion and Conclusions}

The experiments performed revealed interesting trends and results. Due to the lack of repetitions in these experiments, a statistical validity cannot be assigned to the observations.

\subsection{Chloroform/ODE Suspension vs Aqueous Quantum Dots}

Before observing the experimental results, it was important to consider the desired outcomes. When the QDs were suspended in ODE, they had an intensity of roughly 10,000 counts of intensity around the QD fluorescence wavelength. This remained the same when resuspending in $\mathrm{CHCl}_{3}$ Along with this, there was no noticeable UV excitation peak present. This indicates that the QDs were well suspended throughout the solution, both absorbing the UV excitation wavelength of light, and fluorescing. The high intensity of fluorescence (compared to the experimental results) indicates a high number of quantum dots suspended in the solution (compared to the experimental results). If the QDs were suspended as well in the new aqueous solution, the intensities would be proportionate. As the QDs were diluted in the aqueous solution to a 0.3 concentration compared to the $\mathrm{CHCl}_{3}$, a desirable resuspension intensity would be approximately 3,000 counts. The QD control group in the aqueous solution showed an intensity of 100 counts, indicating the QDs were not in solution as well. The highest intensity of any processing method was only 484 counts (peak of full sample, ten phospholipid concentration). While this was an improvement 
over the control group, it remained substantially lower than the desired intensity of 3,000 counts. This indicates that the encapsulation process was not as effective as desired.

\subsection{Full Samples vs. Partial Samples}

Full samples were used as the initial measurements. They showed distinct differences compared to the partial samples. The full samples displayed a wider range of intensities for fluorescence. The signal to noise ratio was noticeably poor at the QD fluorescence wavelength spectrum, and it was difficult to distinguish meaningful trends from in most cases. It did however have a constant greater intensity at the QD fluorescence wavelength spectrum for all samples. This was likely due to there being more QDs throughout the sample.

The partial samples were meant to isolate only the QDs suspended in the aqueous solution, removing any variability introduced from particles that were not well suspended in the solution. It was used as a crude purification process to separate the soluble QDs from the insoluble QDs. The partial samples had a better signal to noise ratio, with a predictable and clear trend in their graphs. They did have a lower intensity than their full sample counterparts. This is likely due to the reduction in QDs available within the sample. With fewer QDs, the QD

fluorescence will be less intense. The partial samples also ran the risk of being a poor representation of the processing method as a whole. It is possible that more or fewer QDs were extracted from the sample by chance during the 
extraction process. This was not a risk in the full samples as all of the sample was transferred.

\subsection{The Superiority of DPPC Phosphocholine Phospholipids}

The DPPC phosphocholine phospholipids appeared to be the superior of the two phospholipids tested. Though the DPPC and DSPC phospholipids appeared similar in the full sample testing, It appeared as though the higher intensities were being reached by the DPPC, while the DSPC had a slightly lower intensity (-50 to -150 counts) overall. The partial sample testing method revealed more obvious differences between the samples. The partial DSPC sample showed no intensity of light within QD fluorescence spectrum. The DPPC showed an increased intensity of fluorescence, greater than that of the QD control group or DSPC. In application this means that the QDCs utilizing DSPC would not be distinguishable from any other substance. Though the UV excitation wavelength in the partial sample with DPPC was nearly three times the intensity of the sample using DSPC, DPPC was still superior. This is because the QD fluorescence was clearly apparent. Not only was it apparent, it was greater than the QD control, indicating that more QDs (or QDCs) were in suspension in the aqueous substance. Because the DPPC tests showed that the QDCs with DPPC had a higher fluorescence at the QD fluorescence, they were considered to be the superior choice for further experimentation despite the fact that they had a higher UV excitation peak in the partial sample. 
One potential cause of the poor performance of the DSPC phospholipids was the longer tail chain length compared to the DPPC counterparts. The longer chains would allow more flexibility and movement of the polar heads of the phospholipids. This would cause more interactions between the polar heads of the phospholipids. With more interactions, and more repulsion between the similarly charged heads, steric hindrance could occur. This would prevent more phospholipids from attaching to the QD surface. In contrast, the shorter chains of the DPPC would have been less free in their movement on the surface of the QD as their short tails would impede how far the polar heads could move. This would allow more phospholipids to attach with less interaction between the polar heads of the phospholipids. This in turn would cause the QD surface to have greater encapsulation in a polar phospholipid head covering. With a more polar encapsulation of individual QDs, QDs would be more readily suspended in an aqueous solution. A representation of this can be seen in Figure 26 


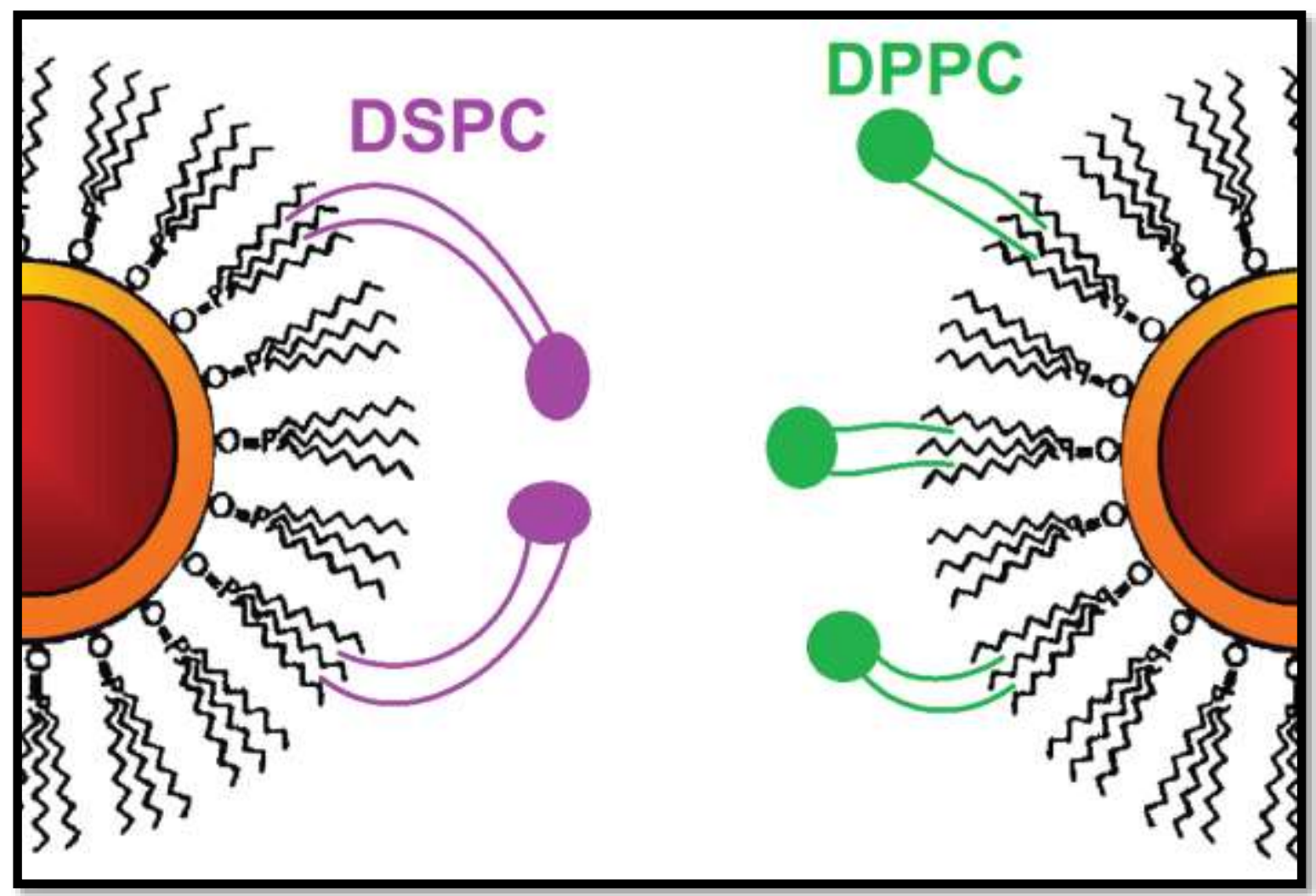

Figure 26: The longer tail chains of DSPC (left) allow for more movement of the polar head of the phospholipid. This may cause more interference between phospholipids when compared to DPPC (right), where the shorter tails restrict movement of the polar heads. (58)

. It is also worth noting that the hydrocarbon chains (tails) of the DPPC are more similar in length to those of the TOP surfactant chains than the DSPC tails. The tail of the DPPC phospholipid has a shorter length than the tail of the DSPC phospholipid. As the agent surrounding the QDs when they are created in ODE is TOP, the chain length of the TOP would hold a large influence on how the QD was coated further. TOP's chemical equation is $\mathrm{P}\left(\mathrm{C}_{8} \mathrm{H}_{17}\right)_{3}$. As the DPPC carbon chain $\left(\mathrm{C}_{40} \mathrm{H}_{80}\right)$ is shorter than DSPC $\left(\mathrm{C}_{44} \mathrm{H}_{88}\right)$, it is of a more similar length to TOP than DSPC. As the chain lengths are more similar, the DPPC may be more likely to bond to the TOP compared to the DSPC. 
It seemed uncharacteristic that the DSPC would have a lower QD fluorescence in the aqueous solution than the QD control. This could have been caused by chance in a single run of the experiment. Further iterations of the experiment may reveal the partial suspension of QDs in the aqueous solution as abnormal.

\subsection{The Ten Phospholipids per Square Nanometer of Quantum Dot Surface Concentration}

Of the three concentrations tested (one DPPC phospholipid per square nanometer of QD, three DPPC phospholipids per square nanometer, and ten DPPC phospholipids per square nanometer), the ten-phospholipid concentration appeared to have the most desirable fluorescence. While this was not especially clear in the full sample, the partial sample revealed that the ten phospholipid concentration appeared to have more QDs suspended in the aqueous solution. The one and three phospholipid concentrations were of similar, lower intensities compared to the ten phospholipid sample. The highest intensity fluorescing sample in the QD spectrum was the ten phospholipid per square nanometer of QD surface concentration.

When observing the UV peaks, a lower intensity is desirable to reduce interference when attempting to detect the QDCs. The worst performing of the QD with DPPC samples in both the full and partial sampling methods was the three-phospholipid concentration. While it had a lower UV peak than the DPPC- 
only control group, had a higher intensity than either of the other two samples. While the one-phospholipid concentration was the lowest in the partial sampling method, it was slightly higher than the ten-phospholipid concentration in the full sample. In both instances, the intensities appeared similar. Because the samples are so similar, it is worth contrasting the UV peak intensity against the QD fluorescence intensity. In the partial samples, the ten-phospholipid concentration sample had a QD fluorescence of $41.6 \%$ intensity compared to the UV peak. The one-phospholipid concentration had a QD fluorescence intensity of $31.4 \%$ of its UV peak. Not only was the overall QD fluorescence intensity of the ten-phospholipid sample greater than the one-phospholipid counterpart, it had a greater ratio of QD fluorescence to UV interference.

The ten DPPC phospholipids per square nanometer concentration seemed to have the most desirable fluorescence. It exhibited the greatest UV fluorescence of all of the samples, as well as the greatest fluorescence in comparison to UV interference.

In the partial samples, there were risks in misrepresenting the whole sample. If the partial sample acted as a reliable, if crude, purification method which was representative of the whole sample, the ten phospholipid concentration was the most desirable of the samples. Further repetitions of the experiment would be useful to determine this. If several repetitions of the full samples were run, the sample values could be averaged, and a trend line fitted to the data. This trend line would more reliably represent the specific 
concentration, and reduce variability. These values could be cross referenced against the trends observed in the partial sample method.

\subsection{Three-DPPC per Quantum Dot Surface Fluorescence}

Though the three-DPPC per square nanometer did prove effective in increasing QD fluorescence compared to the control, it had a larger UV excitation peak than either the one or ten-phospholipid concentration samples. This does not follow a trend relating phospholipid concentration to UV excitation observed. It is possible that the altered concentrations were not well represented in the testing as the phospholipids were not likely to completely dissolve in the pure $\mathrm{CHCl}_{3}$.

\subsection{Areas for Improvement within Current Methods}

There are many aspects of the present methods which would benefit from improvement. Some improvements may not be easily feasible, if at all possible, while others could be done soon. Increasing the number of repetitions on each experiment would reduce potential experimental error, environmental effects, and overall variability. Many of the improvements involve the accuracy of estimating the size of QDs and the corresponding amount of phospholipids.

The calculation for determining the diameter of a QD based on fluorescent wavelength was meant for uncoated CdSe QDs. While the wavelength did shift 
once the QDs were shelled with ZnS, there was not a particular discernable pattern to the shift, nor could it be assumed that the shift would perfectly coincide with what the shift would indicate if the QDs were purely CdSe. The mass of the QDs were similarly regarded as purely CdSe. While it is true that the core, and the majority of the mass is CdSe, it is not in fact pure CdSe. This minor difference was likely overshadowed by the weighing procedures. Small discrepancies in any scale measurement results in major shifts in the number of QDs estimated. Because there are so many QDs, these shifts may not have a major difference in the end result so long as they are kept to a minimum.

The calculation for the phospholipid concentration was also based only the peak's central wavelength. In reality, the QDs were not of one perfectly uniform size, but instead a narrow spectrum of slightly differing sizes. It was assumed that the different sizes were an approximately normal distribution, so the smaller sized QDs would roughly balance with the larger sized QDs when the phospholipid concentration was added.

In the encapsulation procedure, two major changes could benefit the interaction of QDs binding with phospholipids. Changing the resuspension process in resuspending the QDs from ODE into $\mathrm{CHCl}_{3}$ utilizing a cosolvent along with ethanol could prove a more reliable resuspension method. Along with this altered process, the resuspension solution needs to be altered. Methanol can be mixed with chloroform to allow for the phospholipids to dissolve more easily in the solution. Though this may alter the QD dissolving in the solution, improved dissolving of phospholipids is desirable. 


\subsection{Future Work}

Because the shorter chained DPPC appeared to perform better than the DSPC counterpart, it could prove useful to test even shorter chain lengths of phospholipids. As mentioned before, it is important to change the solution into which the phospholipids are being mixed with the QDs in order to promote equal dissolving regardless of the specific phospholipid used.

A more direct method of characterization would be significantly useful in determining if deductions about QD coatings are accurate or reliable. This does not need to be done via imaging. A titration process could be used on the unprocessed QDs and compared to the processed QDs. If the phospholipids have bonded with the surfactant chains the surface charge of the interacting bodies will change. With the change in the surface charge, the $\mathrm{pH}$ of the solution would be altered as well.

There is great potential for future work once an encapsulation procedure has been established. The final aqueous suspension of QDs could be introduced into biological media, such as keratinocyte skin cells, to observe the interaction with cellular tissue, especially potential movement through a cell wall. Beyond this, the QDs could be further functionalized to interact with specific biological markers, such as the markers specific to skin cancer. 


\subsection{Conclusion}

Though there are many potential methods for solubilization and phospholipid functionalization of QDs for use in a hydrophilic medium such as biologic tissue, this thesis refines the previous attempts made at Cal Poly, and introduces new methods. This method continues to hold great potential for cellular tagging, both for cancer cells in the future, as well as for experiments performed at Cal Poly's facility presently. Further characterization and process optimization would be useful before proceeding to those steps. This work has great potential to have an impact on future work at Cal Poly, and beyond. 


\section{LIST OF REFERENCES:}

1. Center for Disease Control. "Cancer." Centers for Disease Control and Prevention. Centers for Disease Control and Prevention, 21 Oct. 2013. Web.

2. American Cancer Society. Cancer Facts \& Figures 2014. Atlanta: American Cancer Society 2014.

3. National Cancer Institute at the National Institutes of Health. "Metastatic Cancer." National Cancer Institute, 28 Mar. 2013. Web.

4. Jemal, Ahmedin, Freddie Bray, Melissa M. Center, Jacques Ferlay, Elizabeth Ward, and David Forman. "Global Cancer Statistics." CA: A Cancer Journal for Clinicians 61.2 (2011): 69-90.

5. American Cancer Society. "What Causes Cancer?" What Causes Cancer? American Cancer Society, 2014. Web.

6. Veenstra, Hidde J., Michel J.w.m. Wouters, Bin B.r. Kroon, Renato. A. Valdés Olmos, and Omgo E. Nieweg. "Less False-negative Sentinel Node Procedures in Melanoma Patients with Experience and Proper Collaboration."Journal of Surgical Oncology 104.5 (2011): 454-57.

7. Pelley, J. L., A. S. Daar, and M. A. Saner. "State of Academic Knowledge on Toxicity and Biological Fate of Quantum Dots." Toxicological Sciences 112.2 (2009): 276-96. Web. 
8. Ratner, B. D. Biomaterials Science: An Introduction to Materials in Medicine. 3rd ed. Amsterdam: Elsevier/Academic, 2013. Print.

9. Marieb, Elaine Nicpon, and Katja Hoehn. Human Anatomy \& Physiology. 9th ed. Boston: Pearson, 2013. Print.

10. OpenStax-CNX. "Layers of the Skin." OpenStax-CNX. N.p., n.d. Web.

11. Mayo Clinic Staff. "Cancer." Definition. Mayo Clinic, 2 Jan. 2014. Web.

12. National Cancer Institute. "Background: Metastatic Cascade." Circulating Tumor Cells for Cancer Diagnosis and Prognosis. National Cancer Institute, n.d. Web.

13. Isaacs, W., and T. Kainu. "Oncogenes and Tumor Suppressor Genes in Prostate Cancer." Epidemiologic Reviews23.1 (2001): 36

41. Www.Cancer.Org. American Cancer Society. Web. <http://www.cancer.org/acs/groups/cid/documents/webcontent/002550pdf.pdf $>$.

14. Skin Cancer Foundation. "Melanoma Causes and Risk Factors." Skin Cancer Foundation. Skin Cancer Foundation, 2014. Web.

15. American Cancer Society. "Ultraviolet (UV) Radiation." American Cancer Society, 2 July 2013. Web.

$<$ http://www.cancer.org/acs/groups/cid/documents/webcontent/acspc039643-pdf.pdf> 
16. American Cancer Society. "Heredity and Cancer." Heredity and Cancer. American Cancer Society, 28 Mar. 2012. Web.

17. MedlinePlus. "Skin Lesion Biopsy: MedlinePlus Medical Encyclopedia." U.S National Library of Medicine. U.S. National Library of Medicine, n.d. Web.

18. Cecil, Russell L., Lee Goldman, and D. A. Ausiello. Cecil Medicine. 23rd ed. Philadelphia: Saunders Elsevier, 2008. Print.

19. American Cancer Society. "Testing Biopsy and Cytology Specimens for Cancer." Cancer.org. American Cancer Society, 7 Mar. 2013. Web. <http://www.cancer.org/acs/groups/cid/documents/webcontent/003185pdf.pdf>.

20. Trotter, MJ, and AK Bruecks. "Interpretation of Skin Biopsies by General Pathologists: Diagnostic Discrepancy Rate Measured by Blinded Review." Archives of Pathology \& Laboratory Medicine 127.11 (2013): 1489-492. Pubmed.gov.

21. Jones, Edward L., Teresa S. Jones, Nathan W. Pearlman, Dexiang Gao, Robert Stovall, Csaba Gajdos, Nicole Kounalakis, Rene Gonzalez, Karl D. Lewis, William A. Robinson, and Martin D. Mccarter. "Long-term Follow-up and Survival of Patients Following a Recurrence of Melanoma After a Negative Sentinel Lymph Node Biopsy Result." JAMA Surgery 148.5 (2013): 456-61. Web. 
22. American Cancer Society. "Tumor Markers." Tumor Markers. American Cancer Society, Web.

23. University of Wisconsin. "Quantum Dots and Nanoparticles." Quantum Dots and Nanoparticles. University of Wisconsin-Madison Materials Research Science and Engineering Center, 2008. Web. $<$ http://education.mrsec.wisc.edu/background/quantum_dots/index.html>.

24. De Leon, Nelson, Ph.D. "The Aufbau Principle." Aufbau Principle. Indiana University Northwest, n.d. Web.

$<$ http://www.iun.edu/ cpanhd/C101webnotes/modernatomictheory/aufbau-principle.html>.

25. Lichtner, Aaron. "Process Development and Characterization of Cadmium Selenide Quantum Dot Synthesis through Nanoparticle Size Optimization." Senior Project 2009. California Polytechnic State University San Luis Obispo.

26. Cortina, E. "Semiconductor Materials: Pn Junction." Department of Nuclear and Particle Physics University of Geneva. University of Geneva, n.d. Web. Apr.-May 2013.

$<$ http://dpnc.unige.ch/tp/elect/doc/02-Semiconductors.pdf>.

27. Strano Research Group. "Exciton Engineering." Exciton Engineering. Massachusetts Institute of Technology, 2013. Web.

28. Rogers, Ben, Jesse Adams, and Sumita Pennathur. Nanotechnology: Understanding Small Systems. 2nd ed. Boca Raton: CRC, 2011. 197-207. Print. 
29. University of Massachusetts Amherst. Introduction to Modern Physics. Digital image. University of Massachusetts Amherst, n.d. Web.

30. Gullapalli, Sravani, and Andrew Barron. "Optical Characterization of Group 12-16 (II-VI) Semiconductor Nanoparticles by Fluorescence Spectroscopy." Connexions. Physical Methods in Chemistry and Nano Science, 26 June 2010. Web.

<http://cnx.org/content/m34656/latest/?collection=col10699/latest>.

31. Lee, Howard. "Quantum Dots." Quantum Dots. Lawrence Livermore National Laboratory, July-Aug. 2000. Web. Apr.-May 2013. $<$ https://www.Ilnl.gov/str/Lee.html>.

32. OSHA. "Safety and Health Topics | Toxic Metals." Safety and Health Topics / Toxic Metals. United States Department of Labor, n.d. Web.

33. Balbus, John M. "Nanoparticles and Health." San Francisco Medicine (2008): 32-33.

34. Hammer, Nathan I., Todd Emrick, and Michael D. Barnes. "Quantum Dots Coordinated with Conjugated Organic Ligands: New Nanomaterials with Novel Photophysics." Nanoscale Research Letters 2.6 (2007): 282-90. Web.

35. Pellegrino, Teresa, Liberato Manna, Stefan Kudera, Tim Liedl, Dmitry Koktysh, Andrey L. Rogach, Simon Keller, Joachim Rädler, Giovanni Natile, and Wolfgang J. Parak. "Hydrophobic Nanocrystals Coated with an Amphiphilic Polymer Shell: A General Route to Water Soluble Nanocrystals." Nano Letters 4.4 (2004): 703-07. Print. 
36. Umm Al Qura University. "PART (2): STRUCTURE AND MODELS OF BIOLOGICAL MEMBRANES." Umm Al Qura University. Umm AI Qura University, n.d. Web.

37. Princeton University. "Phospholipid." Princeton University. Princeton University, n.d. Web.

38. Avanti Polar Lipids. "Avanti Polar Lipids." Avanti Polar Lipids - Discover the Difference... Avanti Polar Lipids, n.d. Web.

39. Esaki, Leo. "Superlattice and Negative Differential Conductivity in Semiconductors." IBM Journal of Research and Development 14 (1970): 61-65. University of Pennsylvania. Web. <http://garfield.library.upenn.edu/classics1987/A1987H916900001.pdf>.

40. University of Illinois at Urbana-Champaign. "Quantum Dots." Web. <http://mechanical.illinois.edu/media/uploads/course_websites/lee_quantu mdots.20100425.4bd4c1c5be8fe7.73485281.pdf>.

41. Murphy, Catherine J., and Jeffery L. Coffer. "Quantum Dots: A Primer." Applied Spectroscopy 56.1 (2002): 16A-27A. Web.

42. Junkin, M., J. Watson, et al. (2009). "Template-Guided Self-Assembly of Colloidal Quantum Dots Using Plasma Lithography." Advanced Materials 21(12): $1247-1251$.

43. Petroff, Pierre M., Axel Lorke, and Atac Imamoglu. "Epitaxially SelfAssembled Quantum Dots." Physics Today 54.5 (2001): 46-52. Web. 
44. Sigma Aldrich. (2007). Advanced Applications of Engineered Nano Particles. Material Matters. 2(1): 10-11. Retrieved from <http://www.sigmaaldrich.com/etc/medialib/docs/Aldrich/Brochure/al_mate rial_matters_v2n1.Par.0001.File.tmp/al_material_matters_v2n1.pdf >

45. InforBarrel Technology. "Quantum Dot Fluorescence." InfoBarrel. InfoBarrel Technology, 13 Nov. 2013. Web.

46. Patel, Prachi. "Colorful Lasers from Q Dots." MIT Technology Review. MIT Technology Review, 29 May 2007. Web.

<http://www.technologyreview.com/news/407971/colorful-lasers-fromqdots/>.

47. Chen, Yanyan, Shenjie Li, Lijian Huang, and Daocheng Pan. "Low-cost and Gram-scale Synthesis of Water-soluble Cu-In-S/ZnS Core/shell Quantum Dots in an Electric Pressure Cooker." Nanoscale 6.3 (2014): 1295. Royal Society of Chemistry. Royal Society of Chemistry. Web. <http://www.rsc.org/suppdata/nr/c3/c3nr05014a/c3nr05014a.pdf>.

48. Ocean Optics. "Ocean Optics - USB4000 Fiber Optic Spectrometer." Ocean Optics. Ocean Optics, Inc., n.d. Web.

49. Pellegrino, Teresa, Liberato Manna, Stefan Kudera, Tim Liedl, Dmitry Koktysh, Andrey L. Rogach, Simon Keller, Joachim Rädler, Giovanni Natile, and Wolfgang J. Parak. "Hydrophobic Nanocrystals Coated with an Amphiphilic Polymer Shell: A General Route to Water Soluble Nanocrystals." Nano Letters 4.4 (2004): 703-07. Web. 
50. Octadecane. (n.d.). G Love: Materials Selection. Retrieved from 141 $<$ http://web.mit.edu/course/3/3.082/www/team2_s02/materials.html>

51. Ophardt, Charles E. "Polarity of Organic Compounds." Polarity of Organic Compounds. Elmhurst College, n.d. Web.

52. Reusch, William. "Lipids." Lipids. Michigan State University, 5 May 2013. Web.

53. Garting, Tommy. (April 2012). Isolation of CdSe Quantum Dot Nanoparticles. < http://education.mrsec.wisc.edu/301.htm>

54. Fisher Scientific. (n.d.). Material Safety Data Sheet: Ethanol, Absolute.

55. Yu, W. William, Lianhua Qu, Wenzhuo Guo, and Xiaogang Peng. "Experimental Determination of the Extinction Coefficient of CdTe, CdSe, and CdS Nanocrystals." Chemistry of Materials 15.14 (2003): 2854-860. Web.

56. Sparks, Laura Christine. "Development and Characterization of Phospholipid Encapsulated Quantum Dot Constructs for Biologic Applications." Master's Thesis 2012. California Polytechnic State University San Luis Obispo

57. Niemczyk, Mark. "Rotary Evaporator Procedure." MPN Organic - Lessons in Organic Chemistry. MPN Organic, n.d. Web.

58. Hélène Bourvon ; Stéphanie Le Calvez ; Hani Kanaan ; Sylvia Meunier Della Gatta ; Cécile Philippot, et al. "On the way to wafer-level quantum dot light-emitting diodes", J. Photon. Energy. 2(1), 021206 (Apr 11, 2012). Web. 


\section{Appendix A1: "SMALL BATCH" CdSe SYNTHESIS PROCEDURE WITH SILICONE OIL BATH FOR HEATING}

The following CdSe/ZnS synthesis protocol and analysis procedure are the standard protocol and procedure documented by Josh Angell and used by Cal Poly's Biomedical Department.

The first part of this procedure outlines 3 different techniques (Purging, Syringing, \& Oil Bath Operation) which are required for quantum dot synthesis. The second part contains instructions to create the selenium/cadmium precursors and then react them to synthesize CdSe quantum dots.

\section{Chemicals}

- 13 mg Cadmium Oxide Powder

- $33 \mathrm{mg}$ Selenium Powder

- $0.6 \mathrm{~mL}$ Oleic Acid (tech grade 90\%)

- $15 \mathrm{~mL}$ Octadecene (tech grade 90\%)

- $0.4 \mathrm{~mL}$ Trioctylphosphine (tech grade 90\%)

- Toluene and Acetone for cleanup

\section{Equipment}

- $2-50 \mathrm{~mL}$ 14/20 1-neck or 3-neck Round Bottom Flasks

- $2-1 \mathrm{~mL}$ Disposable Plastic Syringes

- $2-3 \mathrm{~mL}$ Disposable Plastic Syringes

- $1-5 c c$ Glass Syringe, Luer Lock Tip

- 1 - Veterinary Tip, 18 gauge, 3" SS Needle

- 2 - Small Stir Bars

- 3 to $5-7.5 \mathrm{~mL}$ Borosilicate Vials

- 1 - Medium-Sized Beaker (150 mL)

- 2 to 4 - Rubber Septa

- Hot/Stir Plate with RTD Probe

- Crystallization Dish

- $200 \mathrm{~mL}$ - High Temperature Silicone Oil

- Chemical Spatula

- Kimwipes

- Thermocouple

- Analytical Balance

- Stopwatch

- Latex or Nitrile Gloves 


\section{Chemical Disposal}

All chemicals and equipment used during the quantum dot synthesis must be properly disposed of. Currently there is a vessel for liquid waste and a container for solid wastes such as needles, syringes, kimwipes, etc. which are stored in the yellow, hazardous chemical cabinet in the nanotech lab (Bldg. 41 205).

\section{Preparing Chemical Bottles}

1. Fill a clean dry bottle with the desired chemical.

2. Fit a rubber septum into the top of the bottle.

3. Either purge the solution if it is to be used immediately or store it for later use.

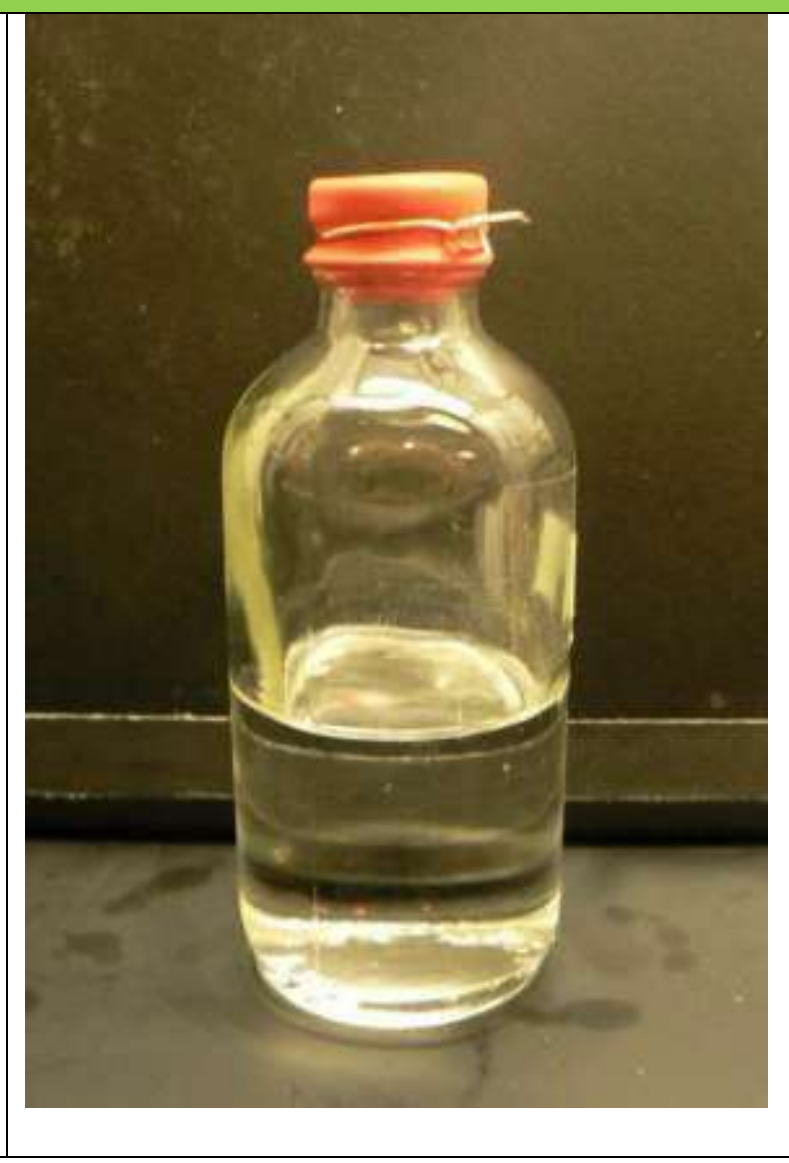




\begin{tabular}{|l|}
\hline \multicolumn{2}{|c|}{ Purging Procedure } \\
This procedure is to be used for any chemical and/or reaction vessel needing \\
purging. \\
is 3 Fill a full. \\
2. Lower $\mathrm{N} 2$ tank gas escape hose \\
into beaker. \\
3. Fully open valve on top of tank. \\
4. Fully open left knob on valve. \\
5. Open middle knob on valve \\
("increase") until bubbles start to \\
appear in the beaker. Maintain this \\
gas flow throughout the procedure. \\
6. Insert N2 purge needle into center \\
of septum. Make sure the needle is \\
above the level of the liquid.
\end{tabular}




7. Insert a vent needle into the
septum next to the $\mathrm{N} 2$ purge
needle. Make sure it is above the
level of the liquid.
8. After inserting the vent needle,
lower the purge needle so that the
tip is submerged in the liquid.
Bubbles should begin to
form in the liquid. If the needle
cannot be submerged below the
level of the liquid, either more
chemical can be added or the bottle
can be carefully tilted.
9. Allow to bubble for at least 10
minutes to remove oxygen from the
chemical.




\section{Syringing Procedure}

This procedure is a method of transferring a chemical from one purged vessel

into another without the introduction of oxygen. All solutions must be properly purged with N2 before using this procedure.

1. Remove a disposable plastic syringe from its packaging.

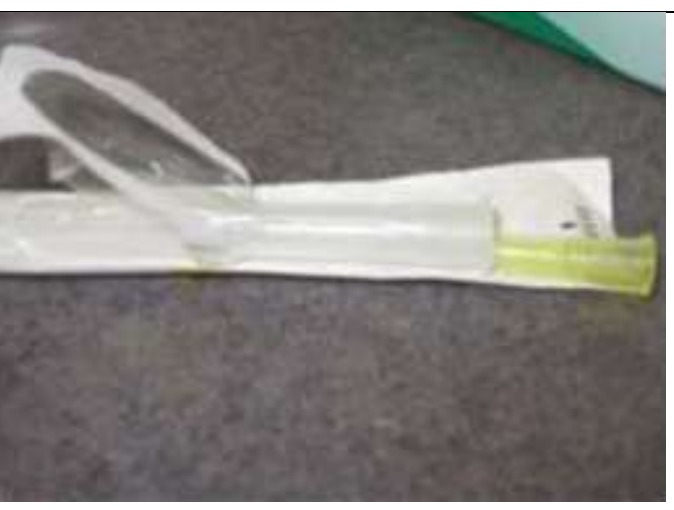

2. Pull the N2 purge needle up so that it is above the level of the liquid. No bubbles should be forming at this point.

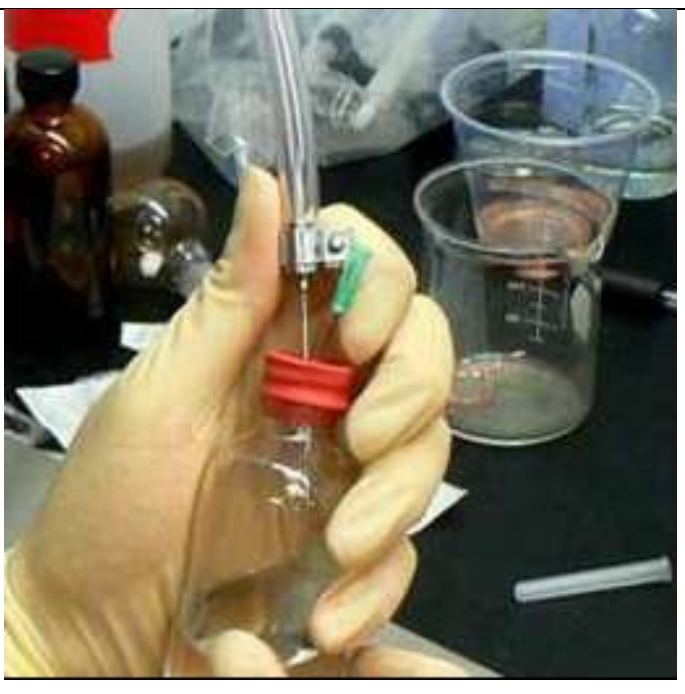




3. Insert the tip of the syringe into
the venting needle.
4. Draw $\mathrm{N} 2$ gas up into the syringe.
5. Remove the N2 filled needle and
syringe from the septum and fully
expel all the $\mathrm{N} 2$ gas by plunging the
syringe down. Make sure to expel
the syringe away from yourself in
the hood.
6. Reinsert the needle and syringe
into the septum.
7. Repeat the filling and expelling
process twice more. This removes
any oxygen in the syringe.
8. Reinsert the needle and syringe
then pull up the syringe to once
again fill the syringe with N2. Do not




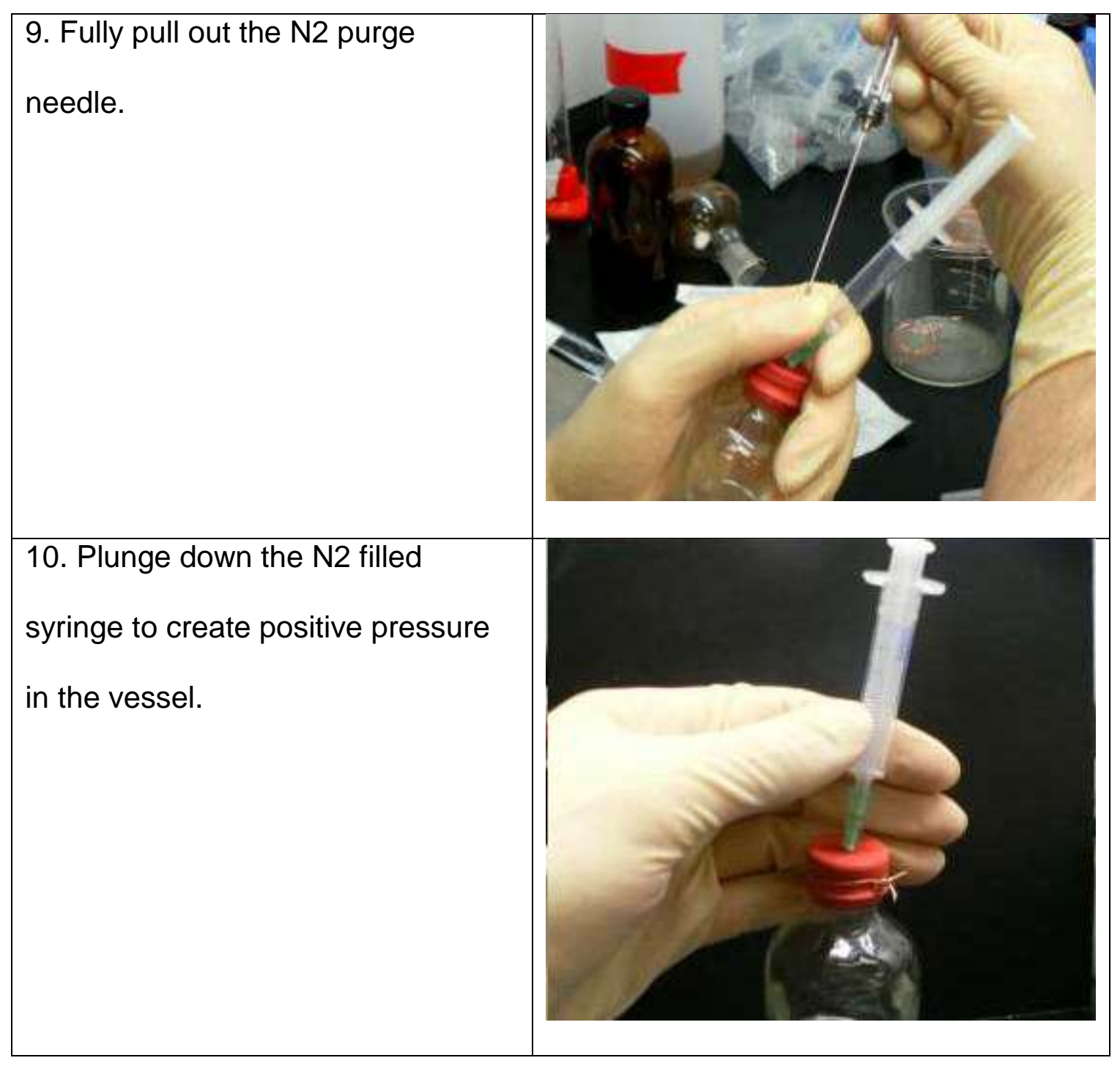




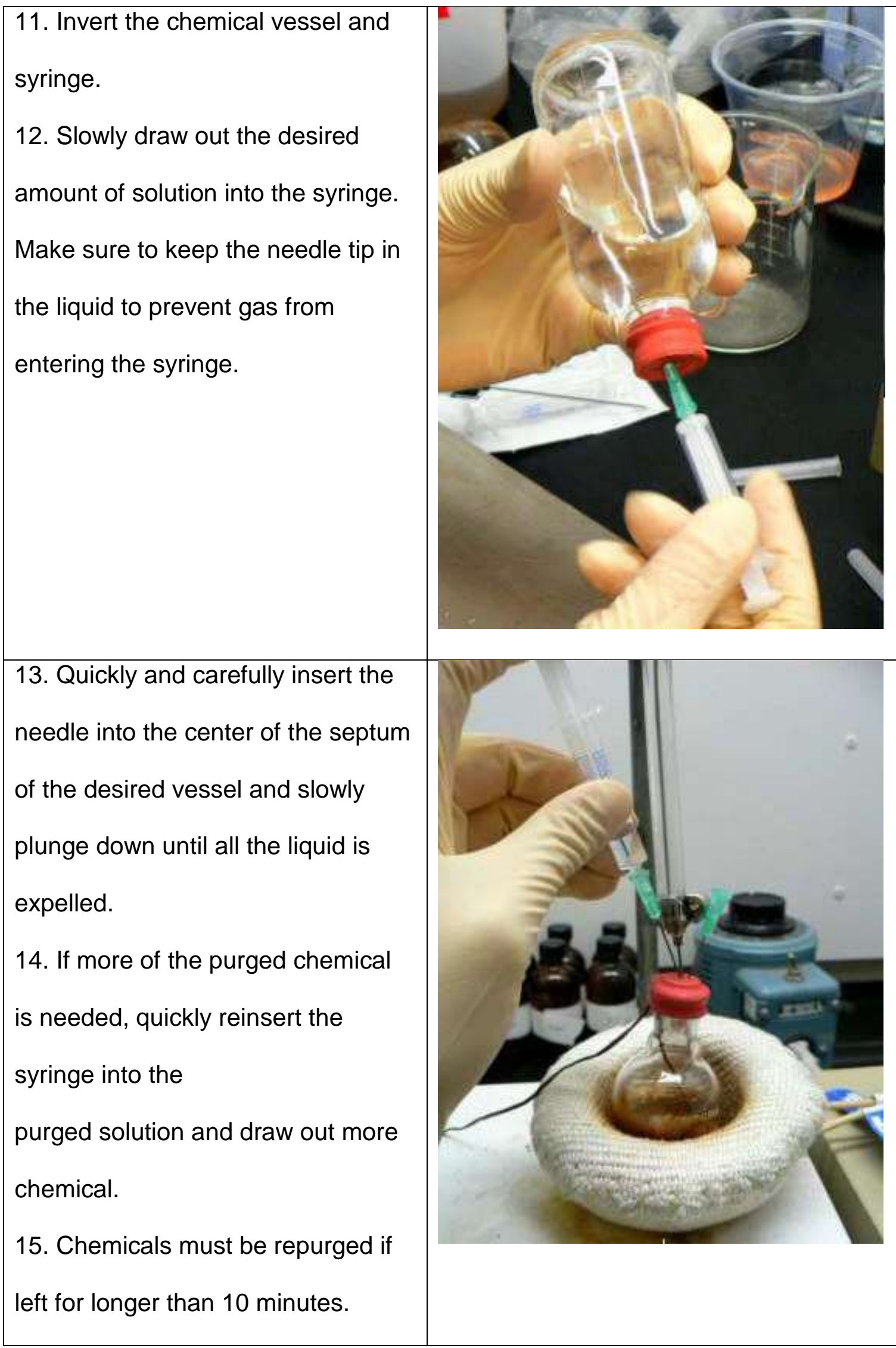




\begin{tabular}{l}
\hline Oil Bath Operation \\
1. Fill crystallization dish with $\sim 200$ \\
2. Place dish on hot plate. \\
3. Put paper clip into dish, which will \\
act as a stir rod for the oil. \\
4. Clamp RTD probe in clamp on \\
ring stand so that it is in the oil bath \\
without touching the glass. \\
5. Press heater button on plate. Set \\
to desired temperature. Allow $\sim 30$ \\
minutes to reach temperature and \\
stabilize. \\
Temperature in flask will be offset \\
from setting on plate by $\sim 17{ }^{\circ} \mathrm{C}$ \\
lower. (i.e. set: $225{ }^{\circ} \mathrm{C}$ flask: $~ 208$ \\
$\left.{ }^{\circ} \mathrm{C}\right)$
\end{tabular}




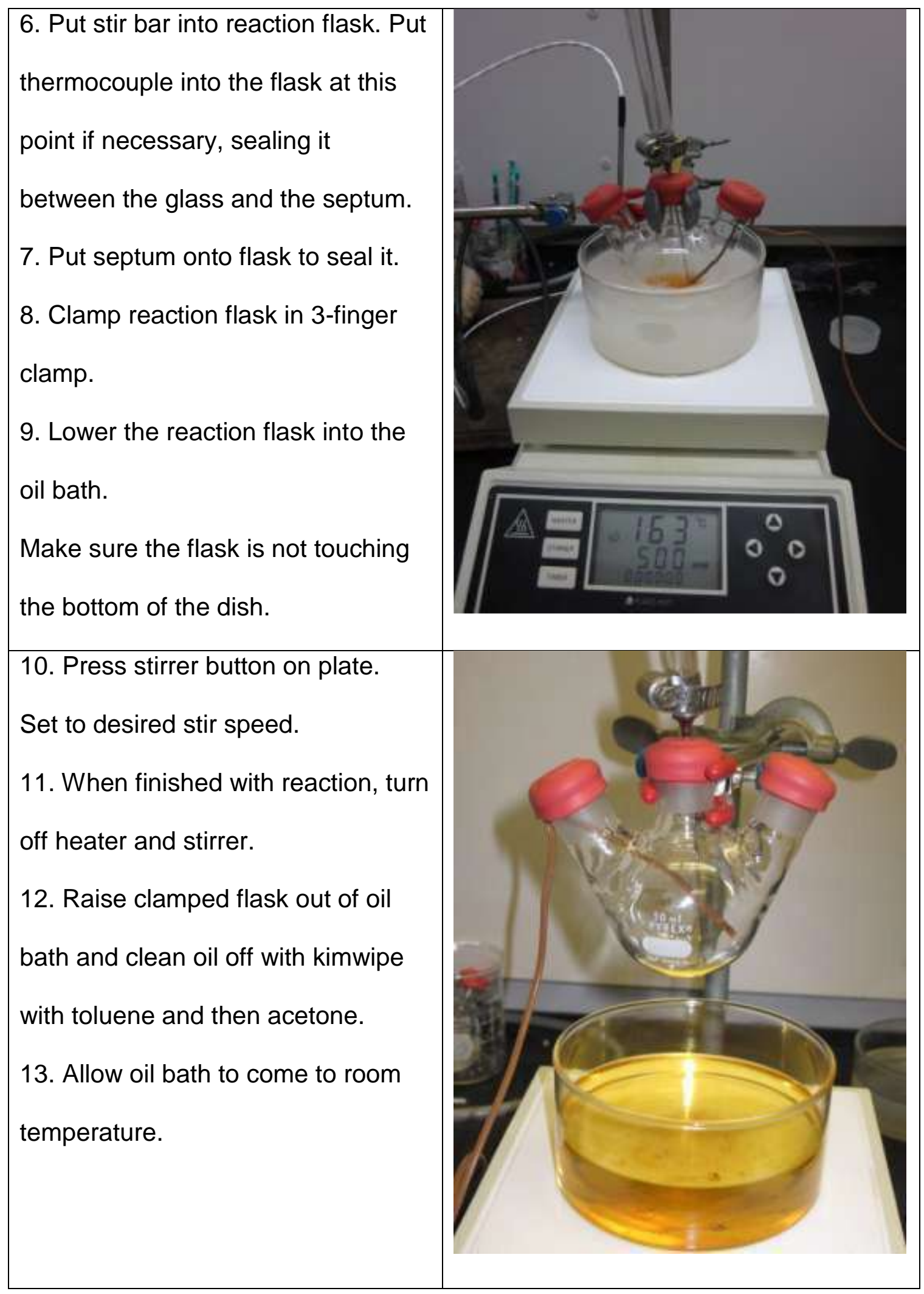




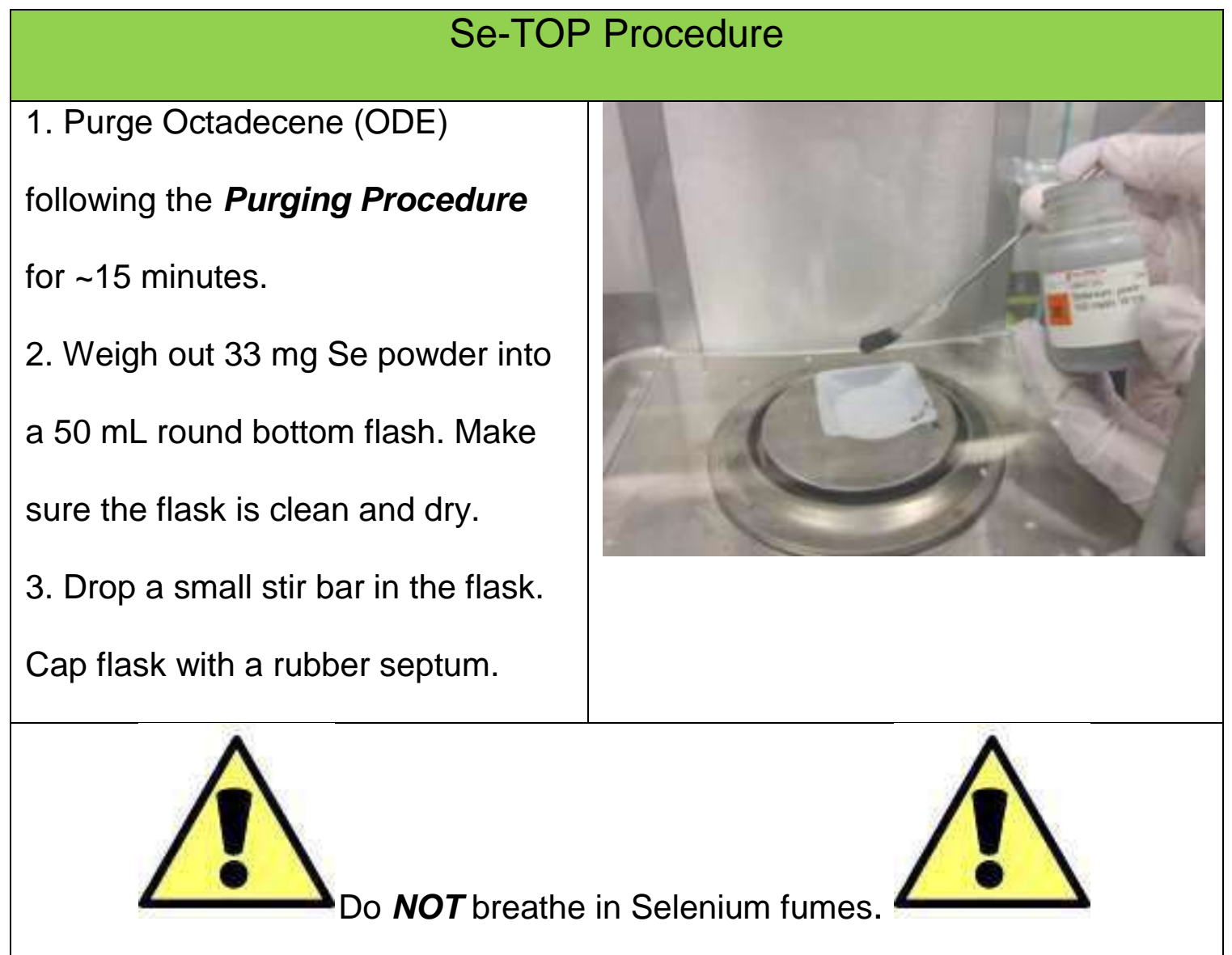

4. Clamp the neck of the flask in a 3-finger clamp on ring stand.

5. Set heat to $150^{\circ} \mathrm{C}$.

6. Purge the flask with N2 gas by inserting the purge needle followed by a venting needle. Allow to purge for 10 minutes.

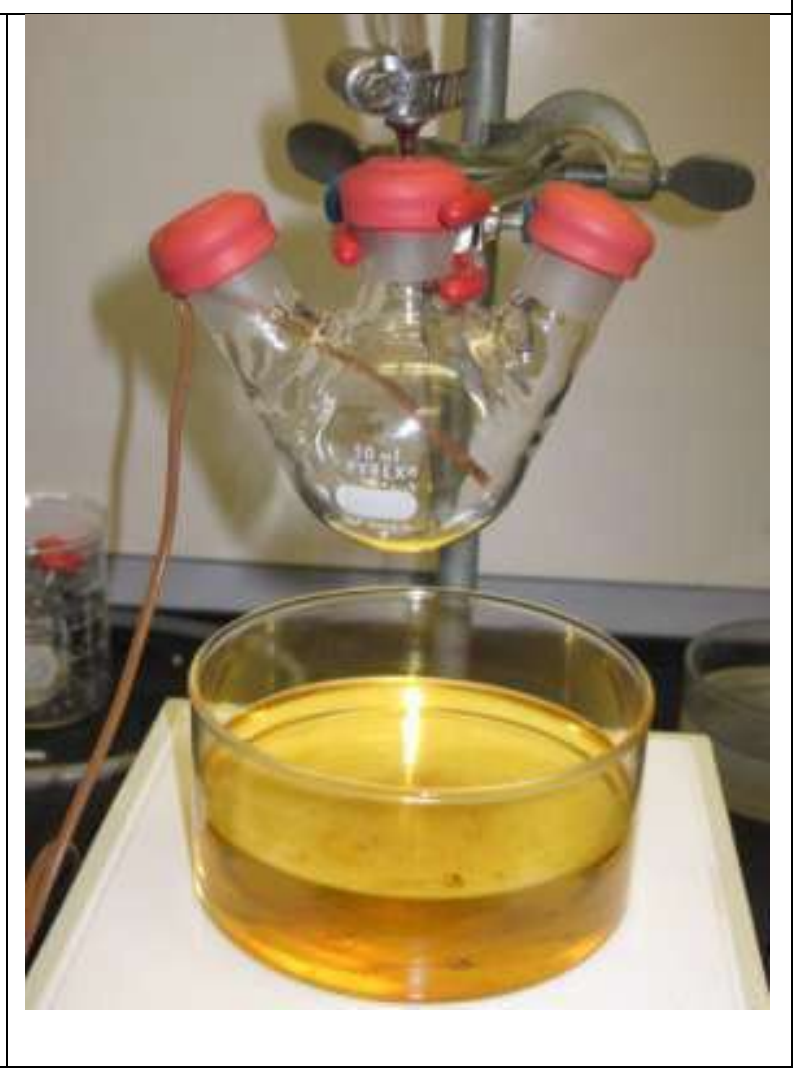




7. Transfer $5 \mathrm{~mL} \mathrm{ODE}$ into the flask
using the Syringing Procedure.
8. Lower flask into oil bath.
9. Set stir to $500 \mathrm{rpm}$.
10. Begin purging trioctylphosphine
using the Purging Procedure.
Purge for 15 minutes.
11. Using the Syringing
Procedure, add
0.4 mL of purged TOP to the Se-
TOP reaction flask. The majority of
the selenium should dissolve
immediately.
12. Continue stirring at $150{ }^{\circ} \mathrm{C}$ until
the solution is completely clear. If all
the selenium does not dissolve, it
may be a sign that oxygen was
introduced into the reaction and
oxidize the TOP before the selenium
and TOP can react.




\begin{tabular}{|l|l|}
\hline $\begin{array}{l}\text { 13. Remove the solution from the oil } \\
\text { bath and pull out the N2 needle and } \\
\text { vent needle. } \\
\text { 14. Once it has cooled, clean the } \\
\text { outside of the flask with toluene and } \\
\text { acetone. This SeTOP solution can } \\
\text { be stored for up to a week and } \\
\text { makes enough SeTOP precursor for }\end{array}$ \\
five QD syntheses. \\
\hline
\end{tabular}




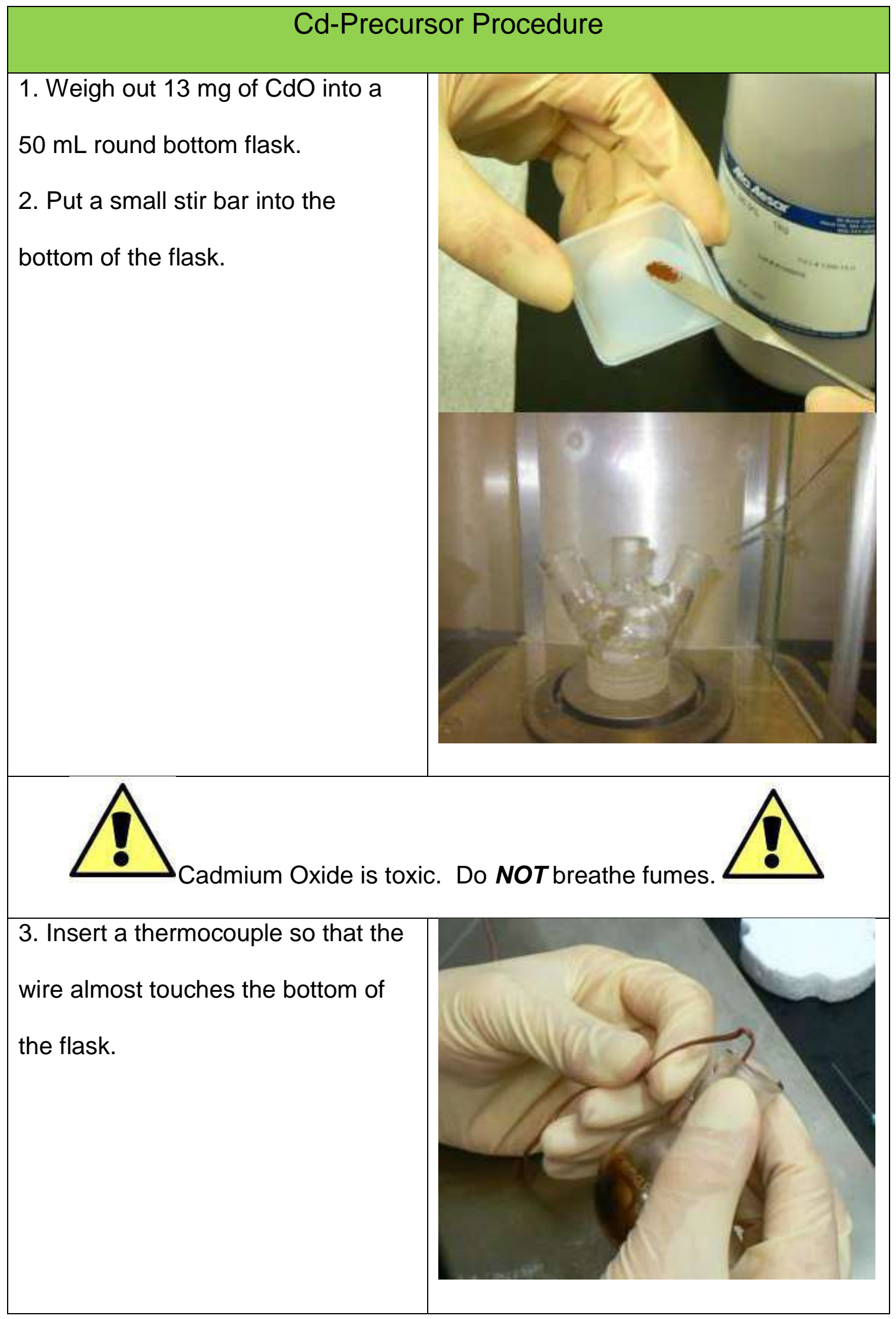




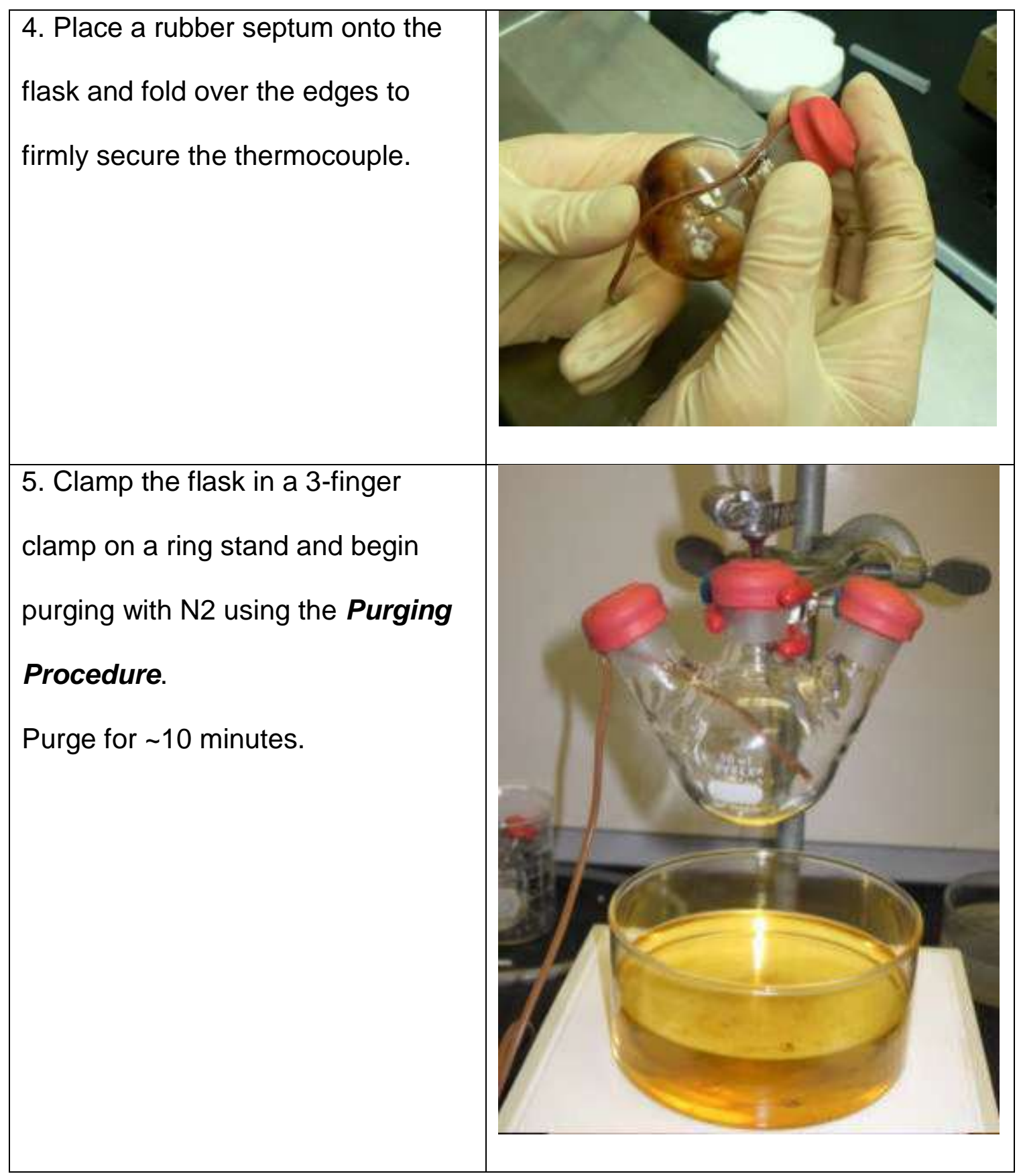




6. Set heat on oil bath to $242^{\circ} \mathrm{C}$
(Adjust as needed to achieve 225
${ }^{\circ} \mathrm{C}$ in flask).
8. Lower the flask into the oil bath.
9. Set the stir function to 500 rpm.
10. Purge the Oleic Acid using the Purging Procedure for 10 minutes.
11. Using the Syringing Procedure, add 0.6 mL Oleic Acid to the Cd reaction
flask.
12. Heat until solution becomes optically clear. CdO has a tendency to stick to
the walls of the flask, so the flask should be agitated occasionally to prevent
build up on walls.
Do NOT breathe fumes.




\section{Sample Removal}

今.

Caution:

This process requires safely handling high-temperature organic, volatile chemicals. Two people must be present and all those present must wear appropriate safety equipment at all times.

\section{Prepare syringes and vials while the Cd precursor is heating.}

1. Attach one metal needle tip securely to a plastic $3 \mathrm{~mL}$ syringe and one to a glass $5 \mathrm{~mL}$ syringe with Luer lock.

2. Remove the caps from clean, dry vials.

3. Once the Cd precursor becomes optically clear, ensure that its temperature is stable at $225^{\circ} \mathrm{C}$ (adjust hot plate setting as needed to achieve stability at 225 $\left.{ }^{\circ} \mathrm{C}\right)$.

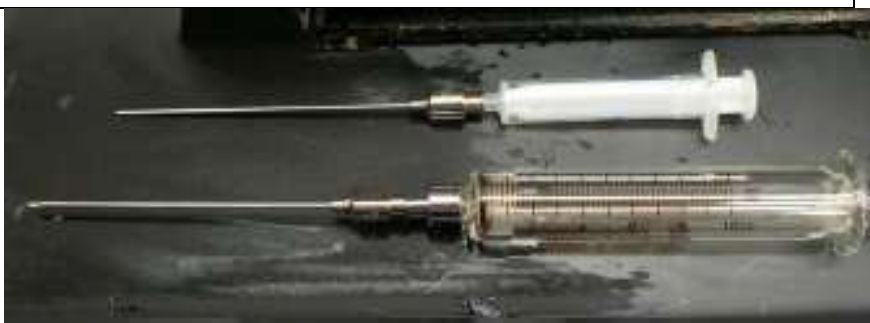




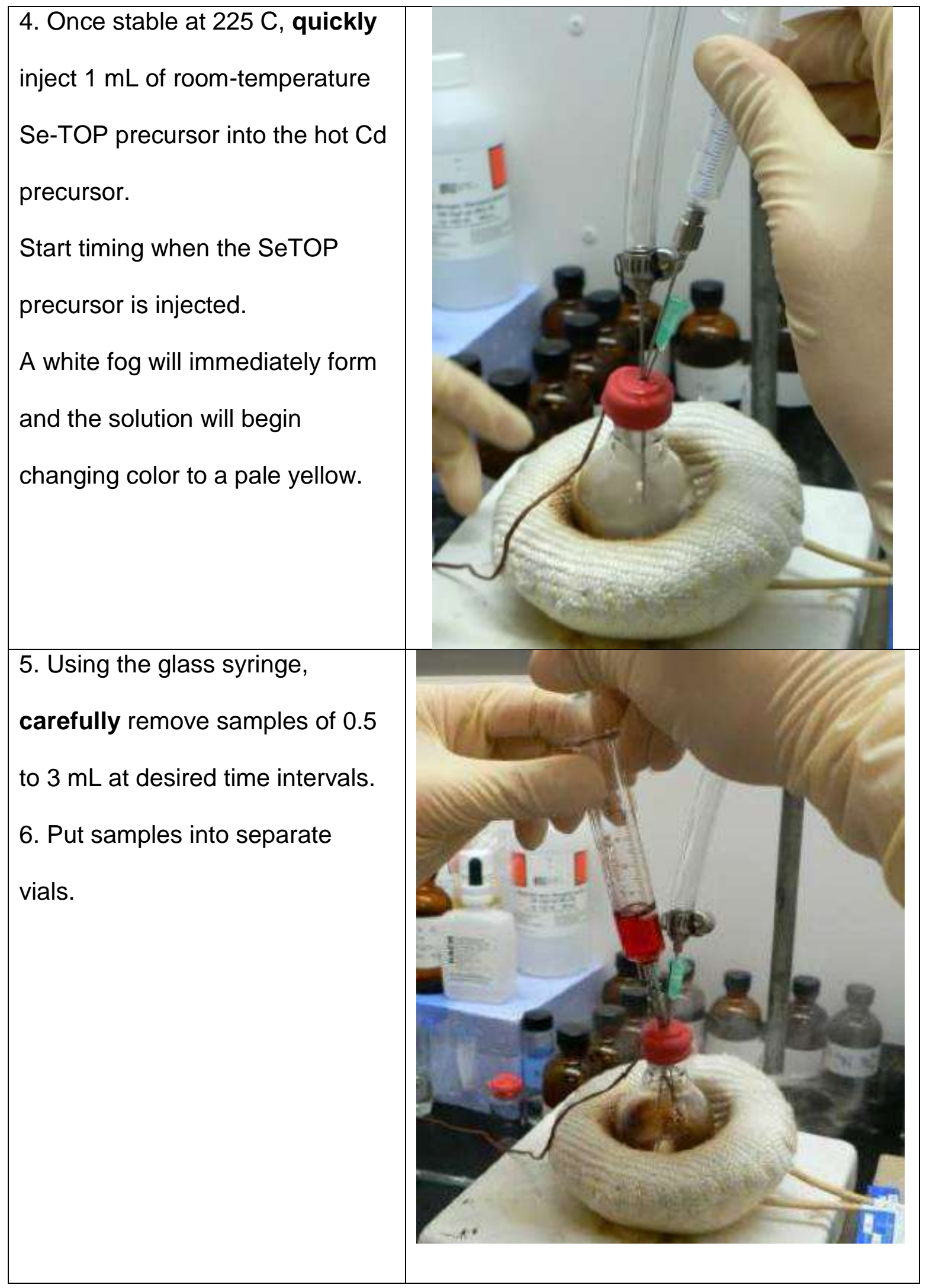




7. Cap and label the vials,
indicating quantum dot type,
date synthesized, reaction
time, and absorbance
wavelength.




\section{Appendix A2: ZnS COATING PROCEDURE}

This procedure follows the CdSe procedure detailed in Appendix A.

The first part of this protocol contains instructions to create the ZnS precursor solution and the second part details how to coat CdSe quantum dots with a $\mathrm{ZnS}$ shell.

\section{Chemicals}

- $1.42 \mathrm{~mL}$ Diethylzinc - ZnEt2 - Solution (1.0M in Heptane)

- $0.22 \mathrm{~mL}$ Hexamethyldisilathiane - (tms)2S - (synthesis grade)

- 5.6mL Tributylphosphine - TBP - (99\% tech grade)

- $1 \mathrm{~mL}$ Butanol

- Toluene and Acetone for cleanup

\section{Equipment}

- 2 - 50mL 14/20 1-neck or 3-neck Round Bottom Flasks (RBF)

- $2-1 \mathrm{~mL}$ Disposable Plastic Syringes

- $2-3 m L$ Disposable Plastic Syringes

- 2 - Small Stir Bars

- 1 - Medium-Sized Beaker

- Hot/Stir Plate with RTD Probe

- Crystallization Dish

- $200 \mathrm{~mL}$ - High Temperature Silicone Oil

- Analytical Balance (accurate to $0.1 \mathrm{mg}$ )

- Silicone and/or Rubber Septa 
- Kimwipes

- Thermocouple

- Borosilicate Vials

- Transfer pipettes

- Stopwatch

- Nitrile Gloves

\section{References}

- Purging procedure - Appendix A1

- $\quad$ Syringing procedure - Appendix A1

- Oil bath operation - Appendix A1

Chemical Disposal
All chemicals and equipment used during the quantum dot synthesis must be
properly disposed of. Currently there is a vessel for liquid waste and a
container for solid wastes such as needles, syringes, kimwipes, etc. which are
stored in the yellow, hazardous chemical cabinet in the nanotech lab (Bldg. 41 -
205).




\section{Chemical Safety}

Many of the chemicals used in this process are toxic and can be very

dangerous if improperly handled. Refer to MSDS for all chemicals prior to

performing this procedure. This procedure must be carried out in a fume hood.

- Tributylphosphine - pyrophoric, toxic

- Diethylzinc - pyrophoric, toxic, corrosive

- Hexamethydisiliathiane - toxic 


\section{ZnS Precursor Solution Procedure}

1. Purge Tributylphosphine (TBP) according to the Purging Procedure for 10 minutes.

2. Drop a stir bar into a $50 \mathrm{~mL}$ round bottom flask.

3. Cap flask with a septum.

This will be the $\mathrm{ZnS}$ vessel.

4. Clamp the flask to the ring stand, suspended above the stir plate.

5. Purge the flask according to Purging Procedure for 10 minutes.

6. Transfer $5.6 \mathrm{~mL}$ of TBP using the Syringing Procedure into the flask.

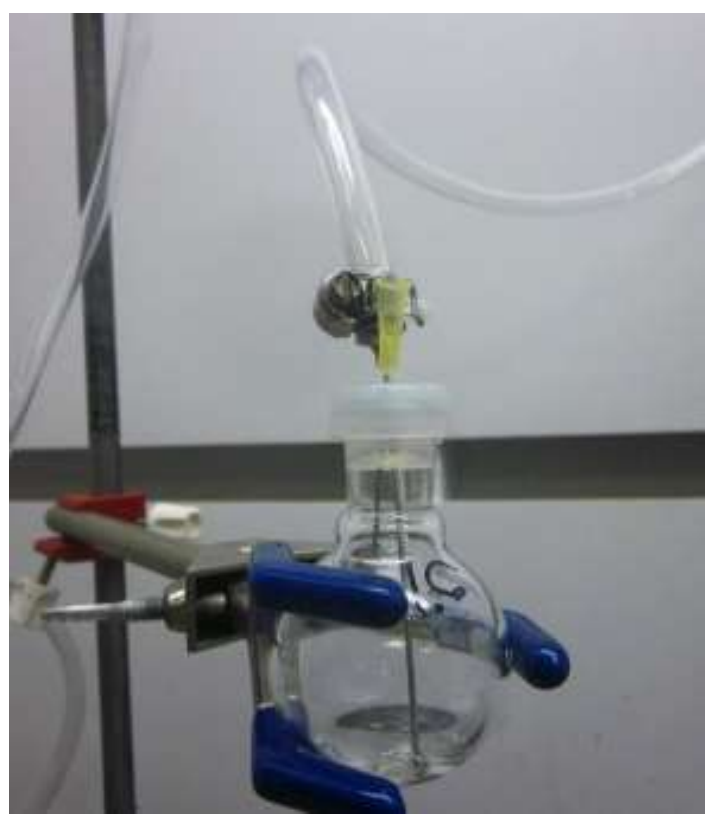


7. Lower N2 needle in flask to below the level of the TBP. Turn the stirring function on the hot plate to $400-500 \mathrm{rpm}$.

9. Purge the ZnEt2 solution using the Purging Procedure for 5 minutes.

10. Transfer $1.42 \mathrm{~mL}$ of $\mathrm{ZnEt} 2$ using the Syringing Procedure into the $\mathrm{ZnS}$ vessel.

11. Continue stirring for 10 to 15 minutes to allow the chemicals to fully dissolve in TBP.

12. Transfer the solution to a vial and cap with a septum.

13. Purge the vial with $\mathrm{N} 2$ for 5 to 10 minutes.

14. Pull out the N2 needle. This solution can be stored for up to one week.

If the solution turns murky or a white precipitate forms, the solution can no longer be used for coating and should be disposed of. 


\section{Coating Procedure}

1. Heat the oil bath to $160^{\circ} \mathrm{C}$ according to the Oil Bath Operation and allow it to stabilize for at least 20 minutes. Turn the stirring function to $400-500 \mathrm{rpm}$ with a paperclip in the oil bath to serve as a flat stir rod.

2. Place a stir bar into a $50 \mathrm{~mL}$ round bottom flask or a 3-necked round bottom flask.

3. Insert a thermocouple so that the wire touches the bottom of the round bottom flask.

4. Place a septum into the neck of the flask and fold the edges down to firmly secure the thermocouple.

5. Clamp the flask to a ring stand and begin purging with $\mathrm{N} 2$ gas using the Purging Procedure.

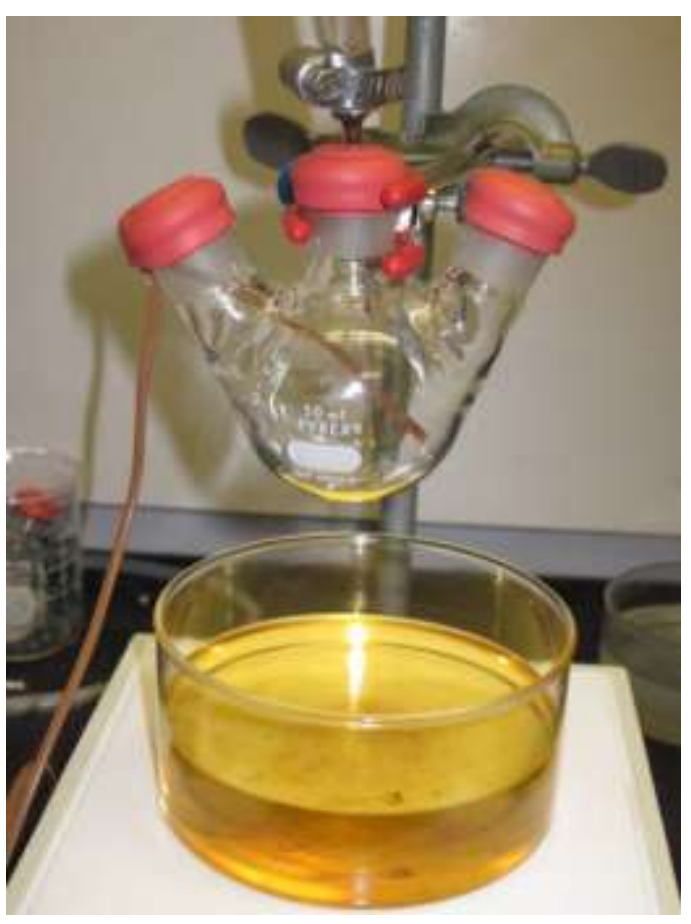


6. Select the desired CdSe quantum dot sample to be coated.

7. Using a $3 \mathrm{~mL}$ disposable plastic syringe, transfer the desired volume of CdSe quantum dots into reaction flask.

The volume of CdSe dots should be between $3 \mathrm{~mL}$ and $10 \mathrm{~mL}$. The procedure does not scale well to volumes greater than $10 \mathrm{~mL}$.

8. Lower the CdSe flask into the oil bath and allow it to heat to $160^{\circ} \mathrm{C}$ and stabilize for 10 minutes.

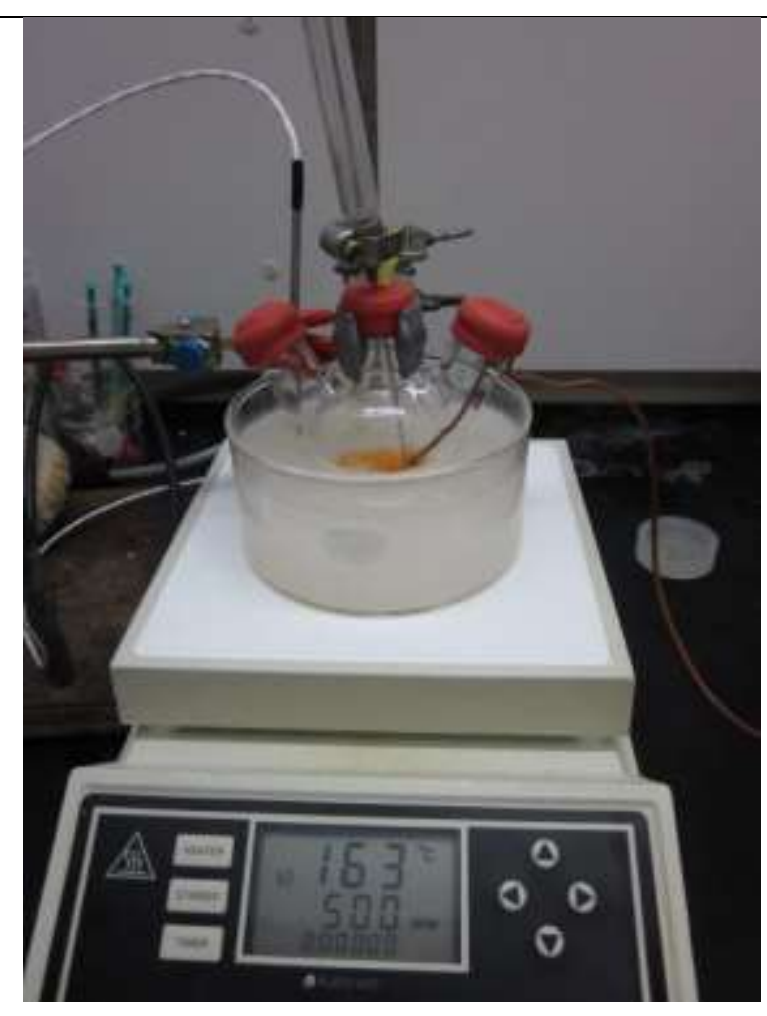


9. While the CdSe solution is heating, purge the ZnS precursor solution with N2 according to the Purging Procedure.

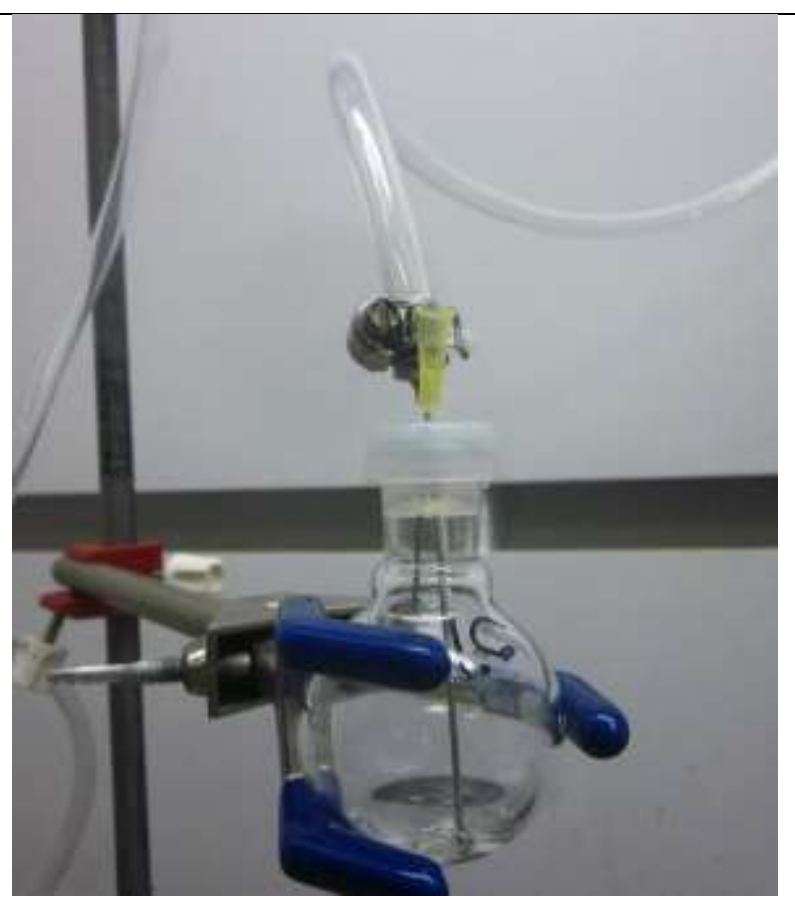

10. Inject the desired volume of ZnS precursor solution dropwise over 2 minutes (according to the concentration of CdSe QDs and coating thickness).

11. Hold the temperature constant at $160^{\circ} \mathrm{C}$ during reaction.

12. The reaction should be allowed to run for 10 minutes.

13. After 10 minutes, raise the flask up out of the oil bath and allow it to cool to $60^{\circ} \mathrm{C}$. 
14. Upon reaching $60^{\circ} \mathrm{C}$, add $1 \mathrm{~mL}$ of butanol to avoid solidification and flocculation.

15. When the solution reaches

room temperature, transfer the

solution to a vial using a transfer

pipette.

16. At this point, a visible change in

fluorescence should be observable

CdSe (left), CdSe-ZnS (right)

when the samples are exposed to

UV light. 


\section{Appendix B: Statistical Analysis of Unshelled to Shelled Quantum Dots}

Paired T-Test and $\mathrm{Cl}$ : unshelled intensity, shelled intensity

Paired T for unshelled intensity - shelled intensity

$\begin{array}{lllll} & \text { N } & \text { Mean } & \text { St Dev } & \text { SE Mean } \\ \text { unshelled intensity } & 6 & 1599 & 739 & 302 \\ \text { shelled intensity } & 6 & 7788 & 2788 & 1138 \\ \text { Difference } & 6 & -6189 & 2565 & 1047\end{array}$

95\% Cl for mean difference: $(-8881,-3497)$

T-Test of mean difference $=0($ vs not $=0): T-$ Value $=-5.91$

P-Value $=0.002$ 